\title{
Irreducible tensor operators in the regular coaction formalisms of compact quantum group algebras
}

\author{
J.F.Cornwell \\ School of Physics and Astronomy, University of St.Andrews, \\ North Haugh, St.Andrews, Fife, KY16 9SS, Scotland, U.K.
}

(23 May 1996)

\begin{abstract}
The defining conditions for the irreducible tensor operators associated with the unitary irreducible corepresentions of compact quantum group algebras are deduced first in both the right and left regular coaction formalisms. In each case it is shown that there are two types of irreducible tensor operator, which may be called 'ordinary' and 'twisted'. The consistency of the definitions is demonstrated, and various consequences are deduced, including generalizations of the Wigner-Eckart theorem for both the ordinary and twisted operators. Also included are discussions (within the regular coaction formalisms for compact quantum group algebras) of inner-products, basis functions, projection operators, Clebsch-Gordan coefficients, and two types of tensor product of corepresentations.

The formulation of quantum homogeneous spaces for compact quantum group algebras is discussed, and the defining conditions for the irreducible tensor operators associated with such quantum homogeneous spaces and with the unitary irreducible corepresentions of the compact quantum group algebras are then deduced. There are two versions, which correspond to restrictions of the right and left regular coactions. In each case it is again shown that there
\end{abstract}


are ordinary and twisted irreducible tensor operators. Various consequences are deduced, including the corresponding generalizations of the Wigner-Eckart theorem.

Typeset using REVTEX 
Running title: Irreducible tensor operators 


\section{INTRODUCTION}

It is well known that most of the applications to physics of the theories of groups and Lie algebras depend on the Wigner-Eckart theorem. It is therefore not surprising that the question of the generalization of this theorem to Hopf algebras having the structure of a

deformation of a Lie algebra has also been the subject of a number of studies ${ }^{1-15}$. The present paper is intended to complement and extend these investigations in various important respects. Its detailed relationship to previous work will be indicated in the appropriate places.

The perspective of the present communication is best introduced by considering matters first in the very well established and familiar context of a compact Lie group $\mathcal{G}$ (c.f. Refs.16,17). Even in this context, one can distinguish three distinct forms of the WignerEckart theorem:

1. The original form ${ }^{18}$ involves the situation in which $\mathcal{G}$ is a group of tranformations that act on an external manifold $\mathcal{M}$, the classic example being the case in which $\mathcal{M}$ is three-dimensional Euclidean space $\Re^{3}$, and $\mathcal{G}$ is the group of all rotations in this space about some fixed point, which may be taken to be the origin $O$ of $\Re^{3}$. Associated with every such rotation $T$ there exists a $3 \times 3$ real orthogonal matrix $\mathbf{R}(T)$, so that the effect of $T$ is to transform each position vector $\mathbf{r}$ into another position vector $\mathbf{r}^{\prime}$, where

$$
\mathbf{r}^{\prime}=\mathbf{R}(T) \mathbf{r}
$$

Also associated with every rotation $T$ is a unitary operator $P(T)$ whose effect on any function $f(\mathbf{r})$ is defined by

$$
P(T) f(\mathbf{r})=f\left(\mathbf{R}(T)^{-1} \mathbf{r}\right)
$$

Let $\Gamma^{p}$ be a unitary irreducible representation of dimension $d_{p}$ of the group $\mathcal{G}$. If there exists a set of functions $\psi_{1}^{p}(\mathbf{r}), \psi_{2}^{p}(\mathbf{r}), \ldots, \psi_{d_{p}}^{p}(\mathbf{r})$ such that 


$$
P(T) \psi_{n}^{p}(\mathbf{r})=\sum_{m=1}^{d_{p}} \Gamma^{p}(T)_{m n} \psi_{m}^{p}(\mathbf{r})
$$

for all $T \in \mathcal{G}$ and all $n=1,2, \ldots, d_{p}$, then these are said to form a set of basis functions for $\boldsymbol{\Gamma}^{p}$. Similarly, if there exists a set of $d_{p}$ operators $Q_{1}^{p}, Q_{2}^{p}, \ldots, Q_{d_{p}}^{p}$ that act on functions $f(\mathbf{r})$ in such a way that

$$
P(T) Q_{n}^{p} P(T)^{-1}=\sum_{m=1}^{d_{p}} \Gamma^{p}(T)_{m n} Q_{m}^{p}
$$

for all $T \in \mathcal{G}$ and all $n=1,2, \ldots, d_{p}$, then these are said to form a set of irreducible tensor operators for $\boldsymbol{\Gamma}^{p}$. Finally, if the inner product for the Hilbert space of functions $f(\mathbf{r})$ is defined by

$$
(f, g)=\int_{-\infty}^{\infty} \int_{-\infty}^{\infty} \int_{-\infty}^{\infty} \overline{f(\mathbf{r})} g(\mathbf{r}) \mathrm{d} x \mathrm{~d} y \mathrm{~d} z
$$

where $\overline{f(\mathbf{r})}$ denotes the complex conjugate of $f(\mathbf{r})$, then the Wigner-Eckart theorem for this situation states that the $j, k$, and $\ell$ dependence of $\left(\psi_{\ell}^{r}, Q_{k}^{q}\left(\phi_{j}^{p}\right)\right)$ depends only on Clebsch-Gordan coefficients for the reduction of the tensor product $\Gamma^{p} \otimes \Gamma^{q}$ into its irreducible constituents $\boldsymbol{\Gamma}^{r}$.

2. In this form the role of the manifold $\mathcal{M}$ is played by $\mathcal{G}$ itself, so that one is concerned with the space of complex-valued continuous functions defined on $\mathcal{G}$. Let this be denoted by $C(\mathcal{G})$. The inner product of $C(\mathcal{G})$ may be taken to be

$$
(f, g)=\int_{\mathcal{G}} \overline{f(T)} g(T) \mathrm{d} T,
$$

where the integral is the left and right invariant normalised Haar integral of $\mathcal{G}$, and $\overline{f(T)}$ is the complex conjugate of $f(T)$. In the right regular formalism, for each $T \in \mathcal{G}$ there exists an operator $\widehat{R}(T)$ that is defined by $\widehat{R}(T) f\left(T^{\prime}\right)=f\left(T^{\prime} T\right)$ for all $f$ and for all $T, T^{\prime} \in \mathcal{G}$. If $f$ is a member of $C(\mathcal{G})$ such that $\widehat{R}(T) f$ spans a finite- dimensional subspace of $C(\mathcal{G})$, then $f$ is said to be a representative function on $\mathcal{G}$. The subspace of $C(\mathcal{G})$ consisting of representative functions will be denoted by $R(\mathcal{G})$. If there exists a set 
of functions $\psi_{1}^{p}(T), \psi_{2}^{p}(T), \ldots, \psi_{d_{p}}^{p}(T)$ such that $\widehat{R}(T) \psi_{n}^{p}\left(T^{\prime}\right)=\sum_{m=1}^{d_{p}} \Gamma^{p}(T)_{m n} \psi_{m}^{p}\left(T^{\prime}\right)$ for all $T, T^{\prime} \in \mathcal{G}$ and all $n=1,2, \ldots, d_{p}$, then these are said to form a set of basis functions for $\Gamma^{p}$. Similarly, if there exists a set of $d_{p}$ operators $Q_{1}^{p}, Q_{2}^{p}, \ldots, Q_{d_{p}}^{p}$ that act on functions $f(T)$ in such a way that $\widehat{R}(T) Q_{n}^{p} \widehat{R}(T)^{-1}=\sum_{m=1}^{d_{p}} \Gamma^{p}(T)_{m n} Q_{m}^{p}$ for all $T \in \mathcal{G}$ and all $n=1,2, \ldots, d_{p}$, then this set is said to form a set of irreducible tensor operators for $\Gamma^{p}$. The Wigner-Eckart theorem for this case states that the $j, k$, and $\ell$ dependence of $\left(\psi_{\ell}^{r}, Q_{k}^{q}\left(\phi_{j}^{p}\right)\right)$ again depends only on Clebsch- Gordan coefficients for the reduction of the tensor product $\Gamma^{p} \otimes \Gamma^{q}$ into its irreducible constituents $\Gamma^{r}$. In the left regular formalism the situation is the same, except only that the operators $\widehat{R}(T)$ are replaced by operators $\widehat{L}(T)$ that are defined by $\widehat{L}(T) f\left(T^{\prime}\right)=f\left(T^{-1} T^{\prime}\right)$ for all $f$ and for all $T, T^{\prime} \in \mathcal{G}$.

3. The final form involves using the abstract carrier spaces of the unitary irreducible representations of $\mathcal{G}$. Let $V^{p}$ be such a carrier space for $\Gamma^{p}$, with ortho-normal basis $\psi_{1}^{p}, \psi_{2}^{p}, \ldots, \psi_{d_{p}}^{p}$, and define for each $T \in \mathcal{G}$ a linear operator $\Phi^{p}(T)$ that acts on $V^{p}$ by the requirement that $\Phi^{p}(T) \psi_{n}^{p}=\sum_{m=1}^{d_{p}} \Gamma^{p}(T)_{m n} \psi_{m}^{p}$ for all $T \in \mathcal{G}$ and all $n=$ $1,2, \ldots, d_{p}$. Let $\boldsymbol{\Gamma}^{p}, \boldsymbol{\Gamma}^{q}$, and $\boldsymbol{\Gamma}^{r}$ be any three unitary irreducible resentations of $\mathcal{G}$. Then one can consider a set of irreducible tensor operators $Q_{1}^{q}, Q_{2}^{q}, \ldots, Q_{d_{q}}^{q}$ that each map $V^{p}$ into $V^{r}$ and which are such that $\Phi^{r}(T) Q_{n}^{q} \Phi^{p}(T)^{-1}=\sum_{m=1}^{d_{q}} \Gamma^{q}(T)_{m n} Q_{m}^{q}$ for all $T \in \mathcal{G}$ and all $n=1,2, \ldots, d_{q}$. In this case the Wigner-Eckart theorem deals with inner products $\langle$,$\rangle defined on V^{r}$ and states that the $j, k$, and $\ell$ dependence of $\left\langle\psi_{\ell}^{r}, Q_{k}^{q}\left(\phi_{j}^{p}\right)\right\rangle$ also depends only on Clebsch-Gordan coefficients for the reduction of the tensor product $\Gamma^{p} \otimes \Gamma^{q}$ into its irreducible constituents $\boldsymbol{\Gamma}^{r}$. In a minor extension of this formalism, one could introduce an inner product space $V$ that is a direct sum of carrier spaces of certain unitary irreducible representations of $\mathcal{G}$ and which contains at least $V^{p} \oplus V^{r}$ (and which, in the extreme case, may contain one carrier space for every inequivalent irreducible representation of $\mathcal{G})$. Then, for each $T \in \mathcal{G}$ an operator $\Phi(T)$ can be defined which maps elements of $V$ into $V$, and which acts as $\Phi^{p}(T)$ on 
$V^{p}$, as $\Phi^{r}(T)$ on $V^{r}$, and so on. The irreducible tensor operators are then required to each map $V$ into $V$ and to be such that $\Phi(T) Q_{n}^{q} \Phi(T)^{-1}=\sum_{m=1}^{d_{q}} \Gamma^{q}(T)_{m n} Q_{m}^{q}$ for all $T \in \mathcal{G}$ and all $n=1,2, \ldots, d_{q}$. In this case the Wigner-Eckart theorem deals with inner products $\langle$,$\rangle defined on V$, but is otherwise the same as above.

The developments that will be described in the present paper up and including Section VIII are essentially within the spirit of the second of these formulations, but deal with a more general Hopf algebra structure. The generalization of the first formulation in terms of quantum homogeneous spaces then follows in Section IX. (It is intended to extend this analysis to the remaining formulation in a subsequent paper).

One most important lesson that can be drawn from these simple group theoretical considerations concerns the consistency of the definitions of the basis functions (or basis vectors) and of the irreducible tensor operators. The essential point will be illustrated in the first of the above formulations, but similar considerations apply in the others. As $P(T) P\left(T^{\prime}\right)=P\left(T T^{\prime}\right)$ and $\boldsymbol{\Gamma}^{p}(T) \boldsymbol{\Gamma}^{p}\left(T^{\prime}\right)=\boldsymbol{\Gamma}^{p}\left(T T^{\prime}\right)$ for all $T, T^{\prime} \in \mathcal{G}$, it follows that if (3) is valid for $T$ and for $T^{\prime}$, then it is also valid for their product $T T^{\prime}$. Similarly, and very significantly, by defining for each $T \in \mathcal{G}$ an operator $\Psi(T)$ by $\Psi(T) Q=P(T) Q P(T)^{-1}$ for every operator $Q$ that acts on functions $f(\mathbf{r})$, the definition (四) can be recast as

$$
\Psi(T)\left(Q_{n}^{p}\right)=\sum_{m=1}^{d_{p}} \Gamma^{p}(T)_{m n} Q_{m}^{p}
$$

for all $T \in \mathcal{G}$ and all $n=1,2, \ldots, d_{p}$. As $\Psi(T) \Psi\left(T^{\prime}\right)=\Psi\left(T T^{\prime}\right)$ for all $T, T^{\prime} \in \mathcal{G}$, it follows that if (7) is valid for $T$ and for $T^{\prime}$, then it is also valid for their product $T T^{\prime}$. Put another way, because of the similarity in form between (3) and (7), the consistency of the definition (四) of the irreducible tensor operators $Q_{n}^{p}$ is ensured by the fact that they too form a basis for a carrier space of $\Gamma^{p}$. In the analysis that follows (cf. Section VI), essentially this argument will be used to justify the definitions that will be given for the irreducible tensor operators of the compact quantum group algebras in the regular corepresentation formalisms, the only essential difference being that the argument has to be cast in terms of corepresentations instead of representations. 
It is well known that the set of functions defined on a Lie group $\mathcal{G}$ form a Hopf algebra, $\mathcal{A}$, and that the dual $\mathcal{A}^{\prime}$ of $\mathcal{A}$ is the universal enveloping algebra of the Lie algebra $\mathcal{L}$ of $\mathcal{G}$. Moreover, the structure of $\mathcal{G}$ can be encoded into the structure of $\mathcal{A}$, and, in particular, $\mathcal{A}$ is commutative. A 'deformation' (or 'quantization') of $\mathcal{A}^{\prime}$ induces a corresponding deformation of $\mathcal{A}$, and will make $\mathcal{A}$ non- commutative as well as being non-cocommutative. Most of the previous work on irreducible tensor operators has been focused on the deformed Hopf algebras $\mathcal{A}^{\prime}$, with $s u_{q}(2)$ receiving the most attention. However, as has been demonstrated by the pioneering work of Woronowicz ${ }^{19-21}$, which itself has been refined and developed by Dijkhuizen and Koornwinder ${ }^{22-26}$, it is of very great interest to produce a self-contained and direct study of generalizations of the Hopf algebras $\mathcal{A}$, which can be done by assuming that they have certain characteristic properties. The resulting structures have been called compact matrix pseudogroups by Woronowicz ${ }^{19-21}$, and compact quantum group algebras by Dijkhuizen and Koornwinder ${ }^{22-26}$. These provide the framework for the present paper, which is devoted to the study of the irreducible tensor operators for compact quantum group algebras. As explained above, this analysis will be given in the regular corepresentation formalisms. (The only previous investigation of irreducible tensor operators within the general compact matrix pseudogroup theory has been by Bragiel $^{5}$, who looked at the analogue of the carrier space formalism (3) above, but with certain restrictive assumptions on multiplicities, though some of the work of Klimyk ${ }^{9}$ involves a discussion of special cases, again in the carrier space formalism).

The structure of the present paper is as follows. Section II contains a brief summary of the essential preliminaries, starting in Subsection II.A with the properties of Hopf *algebras, and continuing in Subsection II.B with the main features of their right comodules. The definition and relevant properties of a compact quantum group algebra $\mathcal{A}$ follow in Subsection II.C. (Of course the developments of Woronowicz of and Dijkhuizen and Koornwinder extend far beyond what is mentioned here, particularly in their their invocation of quantum Tannaka-Krein duality.) This section is concluded in Subsection II.D with some new lemmas concerning the Haar functional of $\mathcal{A}$. The right and left regular comodules 
of $\mathcal{A}$ are described in Section III, and these are employed in Section IV to introduce and develop the concept of basis functions for right corepresentations of $\mathcal{A}$. In Section $\mathrm{V}$ the tensor products (both 'ordinary' and 'twisted') of corepresentations of $\mathcal{A}$ are discussed, along with their associated Clebsch-Gordan coefficients. The heart of the paper is reached in Section VI, where the irreducible tensor operators are defined and some of their immediate properties are deduced. In particular, it will be shown there that in both the right and left regular coaction formulations there are two types of irreducible tensor operators, which will be described as being ordinary and twisted respectively. The motivations for the definitions of Section VI are deliberately relegated to Appendix B in order to emphasize that the treatment of given for the compact quantum group algebras in Sections II to IX is entirely self-contained. In Section VII it is shown that there are two theorems of the Wigner- Eckart type, one for 'ordinary' and one for the 'twisted' irreducible tensor operators. Likewise, in Section VIII, it is demonstrated that these two types of irreducible tensor operator behave differently under multiplication. Finally, in Section IX it is shown how all developments generalize when one considers operators associated with the corresponding homogeneous spaces. In particular, it emerges that there are again two formulations, one associated with the right regular representation and the other with the left regular represntation. The vital algebraic quantity that appears in each version is a $\star$-subalgebra $\mathcal{B}$ of $\mathcal{A}$, which is a right coideal of $\mathcal{A}$ in the right regular formulation, but is a left coideal of $\mathcal{A}$ in the left regular formulation. In Subsection IX.B attention is focused on the right coactions $\pi_{\mathcal{B}}^{R}$ and $\pi_{\mathcal{B}}^{L}$ of $\mathcal{A}$ that are obtained by restricting the right and left regular coactions of $\mathcal{A}$ to its subalgebra $\mathcal{B}$. As these are the transitive $\star$-coactions that correspond to the transitive action of a quantum group on a quantum homogeneous space in the sense of Dijkhuizen and Koornwinder ${ }^{23,24}$, they play the key role in the analysis. In particular the properties of basis functions, as defined in terms of these restricted coactions, are presented in Subsection IX.C, and in Section IX.D the irreducible tensor operators are also defined in terms of these coactions. It is shown there that, associated with both $\pi_{\mathcal{B}}^{R}$ and $\pi_{\mathcal{B}}^{L}$, there are two types of irreducible tensor operator, which are again called ordinary and twisted, and the immediate properties of all 
these irreducible tensor operators are described. In Subsection IX.E it is shown that the irreducible tensor operators satisfy theorems of the Wigner-Eckart type, and the analysis is concluded in Section IX.F with a demonstration that the products of these irreducible tensor operators are themselves expressible as linear combinations of irreducible tensor operators that involve the relevant Clebsch-Gordan coefficients.

Because the space of functions defined on a compact Lie group $\mathcal{G}$ is a special example of a compact quantum group algebra, all the well-known results for compact Lie groups naturally reappear in this particular case. However, as the detailed analysis shows, the theory in the general situation is rather more subtle, and exhibits various complications.

\section{PROPERTIES OF COMPACT QUANTUM GROUP ALGEBRAS}

\section{A. Hopf $*$-algebras}

The purpose of this subsection is mainly to establish notations, and summarize the essential properties. For further details see, for example, Sweedler ${ }^{27}$, Majid ${ }^{28}$, and Chari and Pressley ${ }^{29}$.

A Hopf algebra $\mathcal{A}$ over the field of complex numbers $\mathbb{C}$ is a complex vector space with an identity element $1_{\mathcal{A}}$ that possesses a multiplication operator $M$ (which maps $\mathcal{A} \otimes \mathcal{A}$ into $\mathcal{A}$ ), a unit operator $u$ (which maps $\mathbb{C}$ into $\mathcal{A}$ ), a comultiplication operator $\Delta$ (which maps

$\mathcal{A}$ into $\mathcal{A} \otimes \mathcal{A}$ ), a counit operator $\epsilon$ (which maps $\mathcal{A}$ into $\mathbb{C}^{*}$ ), and an antipode operator $S$ (which maps $\mathcal{A}$ into $\mathcal{A}$ ). These are assumed to be linear in all their arguments and to have the following properties:

$$
\begin{gathered}
M \circ(M \otimes i d)=M \circ(i d \otimes M), \\
(\Delta \otimes i d) \circ \Delta=(i d \otimes \Delta) \circ \Delta, \\
\Delta \circ M=(M \otimes M) \circ(i d \otimes \sigma \otimes i d) \circ(\Delta \otimes \Delta),
\end{gathered}
$$




$$
\begin{gathered}
\epsilon \circ M=M_{\boldsymbol{C}} \circ(\epsilon \otimes \epsilon), \\
M_{\boldsymbol{C}, \mathcal{A}} \circ(\epsilon \otimes i d) \circ \Delta=M_{\mathcal{A}, \boldsymbol{C}} \circ(i d \otimes \epsilon) \circ \Delta=i d, \\
u\left(1_{\boldsymbol{C}}\right)=1_{\mathcal{A}}, \quad \epsilon\left(1_{\mathcal{A}}\right)=1_{\boldsymbol{C}}, \quad S\left(1_{\mathcal{A}}\right)=1_{\mathcal{A}}, \\
M\left(a \otimes 1_{\mathcal{A}}\right)=M\left(1_{\mathcal{A}} \otimes a\right)=a, \text { for all } a \in \mathcal{A}, \\
\Delta\left(1_{\mathcal{A}}\right)=1_{\mathcal{A}} \otimes 1_{\mathcal{A}}, \\
S \circ M=M \circ \sigma \circ(S \otimes S), \\
\Delta \circ S=(S \otimes S) \circ \sigma \circ \Delta, \\
M \circ(S \otimes i d) \circ \Delta=M \circ(i d \otimes S) \circ \Delta=u \circ \epsilon, \\
\epsilon \circ S=\epsilon .
\end{gathered}
$$

Here $\sigma$ is the transposition operator which interchanges the order of its arguments, so that, for example, when acting on $\mathcal{A} \otimes \mathcal{A}, \sigma(a \otimes b)=b \otimes a$ for all $a, b \in \mathcal{A}$. Also $M_{\mathbb{C}}, M_{\mathcal{A}, \boldsymbol{C}}$, and $M_{\mathbb{C}, \mathcal{A}}$ are the mutliplication operators defined by $M_{\mathbb{C}}(w \otimes z)=w z$ for all $w, z \in \mathbb{C}$, and $M_{\mathcal{A}, \boldsymbol{C}}(a \otimes z)=M_{\boldsymbol{C}, \mathcal{A}}(z \otimes a)=z a$ for all $z \in \mathbb{C}$ and all $a \in \mathcal{A}$. The product $M(a \otimes b)$ will sometimes be written more concisely as $a b$, and the coproduct $\Delta$ will sometimes be expressed as

$$
\Delta(a)=\sum_{(a)} a_{(1)} \otimes a_{(2)} .
$$

If $\mathcal{A}$ is finite-dimensional, with basis elements $a_{1}, a_{2}, \ldots$, the structure constants $m_{j k}^{\ell}$, $\mu_{\ell}^{j k}, s_{j}^{k}, \epsilon_{j}$ and $\epsilon^{j}$ may be defined by $M\left(a_{j} \otimes a_{k}\right)=\sum_{\ell} m_{j k}^{\ell} a_{\ell}, \Delta\left(a_{\ell}\right)=\sum_{j, k} \mu_{\ell}^{j k} a_{j} \otimes a_{k}$, $S\left(a_{j}\right)=\sum_{k} s_{j}^{k} a_{k}, \epsilon\left(a_{j}\right)=\epsilon_{j}$, and $1_{\mathcal{A}}=\sum_{j} \epsilon^{j} a_{j}$. Then (8) to (19) imply that 


$$
\begin{aligned}
& \sum_{s} m_{j k}^{s} m_{s \ell}^{t}=\sum_{s} m_{j s}^{t} m_{k \ell}^{s} \\
& \sum_{j} \mu_{\ell}^{j k} \mu_{j}^{s t}=\sum_{j} \mu_{\ell}^{s j} \mu_{j}^{t k} \\
& \sum_{p, q, s, t} \mu_{j}^{p q} \mu_{k}^{s t} m_{p s}^{r} m_{q t}^{u}=\sum_{p} m_{j k}^{p} \mu_{p}^{r u}, \\
& \sum_{\ell} m_{j k}^{\ell} \epsilon_{\ell}=\epsilon_{j} \epsilon_{k} \\
& \sum_{j} \mu_{\ell}^{j k} \epsilon_{j}=\sum_{j} \mu_{\ell}^{k j} \epsilon_{j}=\delta_{\ell}^{k} \\
& \sum_{j} \epsilon^{j} s_{j}^{k}=\epsilon^{k}, \sum_{j} \epsilon^{j} \epsilon_{j}=1_{\boldsymbol{C}}, \\
& \sum_{k} \epsilon^{k} m_{j k}^{\ell}=\sum_{k} \epsilon^{k} m_{k j}^{\ell}=\delta_{j}^{\ell}, \\
& \sum_{j} \epsilon^{j} \mu_{j}^{k \ell}=\epsilon^{k} \epsilon^{\ell} \\
& \sum_{q} m_{j k}^{q} s_{q}^{p}=\sum_{q, r} m_{r q}^{p} s_{j}^{q} s_{k}^{r}, \\
& \sum_{k} \mu_{k}^{p q} s_{j}^{k}=\sum_{k, \ell} \mu_{j}^{k \ell} s_{\ell}^{p} s_{k}^{q} \\
& \sum_{k, \ell, r} \mu_{j}^{k \ell} s_{k}^{r} m_{r \ell}^{t}=\sum_{k, \ell, r} \mu_{j}^{k \ell} s_{\ell}^{r} m_{k r}^{t}=\epsilon_{j} \epsilon^{t}
\end{aligned}
$$

and

$$
\sum_{j} \epsilon_{j} s_{k}^{j}=\epsilon_{k}
$$

A Hopf *-algebra $\mathcal{A}$ is defined to be a Hopf algebra that possesses an additional *operation that maps $\mathcal{A}$ into $\mathcal{A}$. The effect of the $*$ operation on $a \in \mathcal{A}$ will sometimes be denoted by $a^{*}$. In particular 


$$
1_{\mathcal{A}}^{*}=1_{\mathcal{A}}
$$

The other properties are:

$$
\left(* \circ M_{\boldsymbol{C}^{\prime, \mathcal{A}}}\right)(z \otimes a)=M_{\boldsymbol{C}, \mathcal{A}}\left(\bar{z} \otimes a^{*}\right)=\left(* \circ M_{\mathcal{A}, \boldsymbol{C}}\right)(a \otimes z)=M_{\mathcal{A}, \boldsymbol{C}}\left(a^{*} \otimes \bar{z}\right)=\bar{z} a^{*}
$$

(for all $z \in \mathbb{C}$ and all $a \in \mathcal{A}$, where $\bar{z}$ denotes the complex conjugate of $z$ ),

$$
\begin{gathered}
* \circ *=i d, \\
* \circ M=M \circ(* \otimes *) \circ \sigma, \\
\Delta \circ *=(* \otimes *) \circ \Delta, \\
(\epsilon \circ *)(a)=\overline{\epsilon(a)} \text { for all } a \in \mathcal{A}, \\
S \circ * \circ S \circ *=i d,
\end{gathered}
$$

which implies that $S$ is invertible with inverse given by

$$
S^{-1}=* \circ S \circ *
$$

If $\mathcal{A}$ is finite-dimensional, its linear dual will be denoted by $\mathcal{A}^{\prime}$, the prime being used instead of the usual star to avoid any confusion with the $*$-operation that has just been defined. The effect of $a^{\prime} \in \mathcal{A}^{\prime}$ on $a \in \mathcal{A}$ will be denoted by $\left\langle a^{\prime}, a\right\rangle$, and the evaluation map ev (from $\mathcal{A}^{\prime} \otimes \mathcal{A}$ to $\mathbb{C}$ ) will be defined by

$$
e v\left(a^{\prime} \otimes a\right)=\left\langle a^{\prime}, a\right\rangle
$$

for all $a^{\prime} \in \mathcal{A}^{\prime}$ and all $a \in \mathcal{A}$. In the case in which $\mathcal{A}$ is of finite dimension $\mathrm{n}$, the dual basis of $\mathcal{A}^{\prime}$ will be denoted by $a^{1}, a^{2}, \ldots, a^{n}$, and will be assumed to be such that

$$
\left\langle a^{j}, a_{k}\right\rangle=\delta_{k}^{j}
$$

for all $j, k=1,2, \ldots, n$. 


\section{B. Right comodules of Hopf $*$-algebras}

A right $\mathcal{A}$-comodule consists of a vector space $V$ and a linear mapping $\pi_{V}$ from $V$ to $V \otimes \mathcal{A}$ such that

$$
\left(\pi_{V} \otimes i d\right) \circ \pi_{V}=(i d \otimes \Delta) \circ \pi_{V}
$$

and

$$
M_{V, \boldsymbol{C}} \circ(i d \otimes \epsilon) \circ \pi_{V}=i d
$$

where $M_{V, \boldsymbol{C}}(v \otimes z)=z v$ for all $v \in V$ and all $z \in \mathbb{C}$. The operation $\pi_{V}$ is then said to be a right coaction and provides a corepresentation of $\mathcal{A}$ with carrier space $V$. The present subsection will be devoted to a very brief account of the essential features of the corepresentations of $\mathcal{A}$. (For the intimate connection between the corepresentation theory of $\mathcal{A}$ and the representation theory of $\mathcal{A}^{\prime}$, see Appendix A.)

If $V$ is of finite dimension $d$, with basis $v_{1}, v_{2}, \ldots, v_{d}$, then there exists a uniquely deter-

mined set of elements $\pi_{j k}^{V}$ of $\mathcal{A}$ (for $j, k=1,2, \ldots, d$ ), called the matrix coefficients of $\pi_{V}$, which are such that

$$
\pi_{V}\left(v_{j}\right)=\sum_{k=1}^{d} v_{k} \otimes \pi_{k j}^{V}
$$

for all $j=1,2, \ldots, d$. (In this situation the corepresentation is said to have dimension $d$ ). The requirements (43) and (44) then imply that

$$
\Delta\left(\pi_{j k}^{V}\right)=\sum_{\ell=1}^{d} \pi_{j \ell}^{V} \otimes \pi_{\ell k}^{V}
$$

and

$$
\epsilon\left(\pi_{j k}^{V}\right)=\delta_{j k}
$$

(for $j, k=1,2, \ldots, d$ ). It is sometimes convenient to write

$$
\pi_{V}(v)=\sum_{[v]} v_{[1]} \otimes v_{[2]}
$$


where $v_{[1]} \in V$ and $v_{[2]} \in \mathcal{A}$.

Two right $\mathcal{A}$-comodules, with carrier spaces $V$ and $W$, coactions $\pi_{V}$ and $\pi_{W}$, and matrix coefficients $\pi_{j k}^{V}$ and $\pi_{j k}^{W}$, are said to be equivalent if there exists a one-to-mapping $\Phi$ from $V$ to $W$ such that

$$
\pi_{W} \circ \Phi=(\Phi \otimes i d) \circ \pi_{V} .
$$

If $V$ and $W$ have bases $v_{1}, v_{2}, \ldots, v_{d}$ and $w_{1}, w_{2}, \ldots, w_{d}$ respectively, then to the mapping $\Phi$ there corresponds a $d \times d$ non-singular matrix $\boldsymbol{\Phi}$ such that

$$
\sum_{\ell=1}^{d} \Phi_{j \ell} \pi_{\ell k}^{V}=\sum_{\ell=1}^{d} \pi_{j \ell}^{W} \Phi_{\ell k}
$$

for all $j, k=1,2, \ldots, d$.

A subspace $W \subset V$ is said to be invariant under $\pi_{V}$ if $\pi_{V}(w) \subset W \otimes \mathcal{A}$ for all $w \in W$, and a corepresentation is described as being irreducible if $V$ and 0 are the only invariant subspaces of $V$. If $V$ is the direct sum of two invariant subspaces of $V$, then the corepresentation $\pi_{V}$ is said to be completely reducible.

If $V$ is endowed with an inner product $\langle,\rangle_{V}$ (such that $\left\langle z w, z^{\prime} v\right\rangle_{V}=\bar{z} z^{\prime}\langle w, v\rangle_{V}$ for all $z, z^{\prime} \in \mathbb{C}^{\prime}$ and all $\left.v, w \in V\right)$, then $\pi_{V}$ is said to give a unitary corepresentation if

$$
\sum_{[v]}\left\langle w, v_{[1]}\right\rangle_{V} S\left(v_{[2]}\right)=\sum_{[w]}\left\langle w_{[1]}, v\right\rangle_{V} w_{[2]}^{*}
$$

for all $v, w \in V$. It can be $\operatorname{shown}^{22-26}$ that if $v_{1}, v_{2}, \ldots, v_{d}$ is an ortho-normal basis of $V$ then

$$
\begin{gathered}
S\left(\pi_{j k}^{V}\right)=\pi_{k j}^{V *}, \\
\sum_{\ell=1}^{d} M\left(\pi_{\ell j}^{V *} \otimes \pi_{\ell k}^{V}\right)=\delta_{j k} 1_{\mathcal{A}},
\end{gathered}
$$

and

$$
\sum_{\ell=1}^{d} M\left(\pi_{j \ell}^{V} \otimes \pi_{k \ell}^{V *}\right)=\delta_{j k} 1_{\mathcal{A}}
$$


(for all $j, k=1,2, \ldots, d)$.

Corresponding to a right $\mathcal{A}$-comodule with carrier space $V$ and coaction $\pi_{V}$ from $V$ to $V \otimes \mathcal{A}$ there exist two other right $\mathcal{A}$-comodules formed from the same carrier space. Firstly, there is the coaction $\pi_{V}^{\ddagger}$, which is said to be doubly contragredient to $\pi_{V}$, and which is defined (as a mapping from $V$ to $V \otimes \mathcal{A}$ ) by

$$
\pi_{V}^{\ddagger}=\left(i d \otimes S^{2}\right) \circ \pi_{V}
$$

With the matrix coefficients $\pi_{j k}^{V \ddagger}$ of $\pi_{V}^{\ddagger}$ being defined by

$$
\pi_{V}^{\ddagger}\left(v_{j}\right)=\sum_{k=1}^{d} v_{k} \otimes \pi_{k j}^{V \ddagger}
$$

for all $j=1,2, \ldots, d$, it follows from (45) that

$$
\pi_{j k}^{V \ddagger}=S^{2}\left(\pi_{j k}^{V}\right)
$$

for all $j, k=1,2, \ldots, d$. Secondly, let $\bar{V}$ be the conjugate space to $V$ (so that as an Abelian group $\bar{V}$ is isomorphic to $V$, but the scalar multiplication operator $M_{\mathbb{C}, \bar{V}}$ for $\bar{V}$ is defined in terms of the corresponding operator $M_{\mathbb{C}^{\prime}, V}$ for $V$ by $\left.M_{\mathbb{C}, \bar{V}}(z \otimes v)=M_{\mathbb{C}, V}(\bar{z} \otimes v)\right)$. Then the coaction $\bar{\pi}_{\bar{V}}$, which is said to be conjugate to $\pi_{V}$, is defined (as a mapping from $\bar{V}$ to $\bar{V} \otimes \mathcal{A}$ ) by

$$
\bar{\pi}_{\bar{V}}=(i d \otimes *) \circ \pi_{V},
$$

so its matrix coefficients $\bar{\pi} \bar{V}$ are given by

$$
\bar{\pi}_{j k}^{\bar{V}}=\pi_{j k}^{V *}
$$

for all $j, k=1,2, \ldots, d$.

\section{Compact quantum group algebras}

A compact quantum group algebra (or CQG algebra for short) may be defined ${ }^{22-26}$ as a Hopf $*$-algebra that is spanned by the matrix coefficients of its non- equivalent finitedimensional unitary irreducible corepresentations. Koornwinder and Dijkhuizen ${ }^{22-26}$ have 
shown that if $\mathcal{A}$ is a CQG algebra then every finite-dimensional corepresentation of $\mathcal{A}$ is equivalent to a unitary corepresentation, and that every finite-dimensional reducible corepresentation of $\mathcal{A}$ is completely reducible. Moreover ${ }^{22-26}$ if $\mathcal{A}$ is a CQG algebra then $\mathcal{A}$ possesses a Haar functional, $h$, which is a mapping of $\mathcal{A}$ into $\mathbb{C}$ such that

$$
\begin{gathered}
h\left(1_{\mathcal{A}}\right)=1_{\boldsymbol{C}}, \\
h\left(M\left(a^{*} \otimes a\right)>0,\right. \\
h\left(a^{*}\right)=\overline{h(a)}, \\
h(S(a))=h(a),
\end{gathered}
$$

and

$$
\left(M_{\mathbb{C}^{\prime}, \mathcal{A}} \circ(h \otimes i d) \circ \Delta\right)(a)=\left(M_{\mathcal{A}, C^{b}} \circ(i d \otimes h) \circ \Delta\right)(a)=h(a) 1_{\mathcal{A}}
$$

for all $a \in \mathcal{A}$.

Koornwinder and Dijkhuizen ${ }^{22-26}$ have also shown that if $\pi^{p}$ and $\pi^{q}$ are two nonequivalent irreducible corepresentations of a CQG algebra $\mathcal{A}$ with dimensions $d_{p}$ and $d_{q}$ and matrix coefficients $\pi_{j k}^{p}$ and $\pi_{m n}^{q}$ respectively, then

$$
h\left(M\left(\pi_{j k}^{p} \otimes S\left(\pi_{m n}^{q}\right)\right)=0, \quad h\left(M\left(S\left(\pi_{j k}^{p}\right) \otimes \pi_{m n}^{q}\right)=0\right.\right.
$$

(for all $j, k=1,2, \ldots, d_{p}$ and for all $m, n=1,2, \ldots, d_{q}$ ). Moreover every $\pi^{p}$ irreducible corepresentation of $\mathcal{A}$ is equivalent to its doubly contragredient partner $\pi^{p \ddagger}$, so in each such case there exists a non-singular $d_{p} \times d_{p}$ matrix $\mathbf{F}^{p}$ such that

$$
\sum_{k=1}^{d_{p}} F_{j k}^{p} \pi_{k \ell}^{p}=\sum_{k=1}^{d_{p}} \pi_{j k}^{p \ddagger} F_{k \ell}^{p}
$$

(for all $j, \ell=1,2, \ldots, d_{p}$ ). Then, if $\pi^{p}$ is a unitary irreducible corepresentation of $\mathcal{A}$,

$$
h\left(M\left(\pi_{j k}^{p} \otimes S\left(\pi_{m n}^{p}\right)\right)\right)=\delta_{j n} F_{m k}^{p} / \operatorname{tr}\left(\mathbf{F}^{p}\right),
$$


and

$$
h\left(M\left(S\left(\pi_{j k}^{p}\right) \otimes \pi_{m n}^{p}\right)\right)=\delta_{j n}\left(\left(\mathbf{F}^{p}\right)^{-1}\right)_{m k} / \operatorname{tr}\left(\left(\mathbf{F}^{p}\right)^{-1}\right)
$$

(for all $\left.j, k, m, n=1,2, \ldots, d_{p}\right)$.

Of course in the special case in which $\mathcal{A}$ is the space of functions defined on a compact group $\mathcal{G}, \mathcal{A}$ is commutative (i.e. $M=M \circ \sigma), S^{2}=i d, h$ is the Haar integral

$$
h(a)=\int_{\mathcal{G}} a(x) \mathrm{d} x
$$

and (64) express the invariance properties

$$
\int_{\mathcal{G}} a(y x) \mathrm{d} x=\int_{\mathcal{G}} a(x) \mathrm{d} x=\int_{\mathcal{G}} a(x y) \mathrm{d} x
$$

(for all $y \in \mathcal{G}$ ). Moreover in this case each corepresentation of $\mathcal{A}$ is identical to its doubly contragredient partner, and (65), (67), and (68) correspond to the orthogonality theorems for the unitary irreducible representations of $\mathcal{G}$.

\section{Lemmas concerning the Haar functional}

It will now be shown that

$$
\sum_{(b)} h\left(M\left(a \otimes b_{(1)}\right)\right) S\left(b_{(2)}\right)=\sum_{(a)} h\left(M\left(a_{(1)} \otimes b\right)\right) a_{(2)}
$$

for all $a, b \in \mathcal{A}$.

To prove this consider the operation $u \circ h \circ M$. As the left-hand equality of (64) can be re-expressed as

$$
M_{\mathbb{C}, \mathcal{A}^{\prime}} \circ(h \otimes i d) \circ \Delta=u \circ h
$$

it follows from (10) that

$$
u \circ h \circ M=M_{C^{\prime}, \mathcal{A}} \circ(h \otimes i d) \circ(M \otimes M) \circ(i d \otimes \sigma \otimes i d) \circ(\Delta \otimes \Delta) .
$$

However, by (12), (18), and (9), it also follows that 


$$
u \circ h \circ M=M_{\boldsymbol{C}, \mathcal{A}} \circ(h \otimes M) \circ(M \otimes S \otimes i d) \circ(i d \otimes \Delta \otimes i d) \circ(i d \otimes \Delta) .
$$

Comparing (73) and (74) then gives

$$
\begin{aligned}
& M_{\boldsymbol{C}, \mathcal{A}} \circ(h \otimes M) \circ(M \otimes S \otimes i d) \circ(i d \otimes \Delta \otimes i d) \circ(i d \otimes \Delta) \\
& =M_{\boldsymbol{C}, \mathcal{A}} \circ(h \otimes i d) \circ(M \otimes M) \circ(i d \otimes \sigma \otimes i d) \circ(\Delta \otimes \Delta) .
\end{aligned}
$$

which can be re-expressed as

$$
M \circ(U \otimes i d) \circ(i d \otimes \Delta)=M \circ(V \otimes i d) \circ(i d \otimes \Delta),
$$

where

$$
U=M_{\boldsymbol{C}, \mathcal{A}} \circ(h \otimes i d) \circ(M \otimes i d) \circ(i d \otimes \sigma) \circ(\Delta \otimes i d)
$$

and

$$
V=M_{\mathbb{C}, \mathcal{A}} \circ(h \otimes S) \circ(M \otimes i d) \circ(i d \otimes \Delta) .
$$

However, (75) implies that

$$
\begin{aligned}
& M \circ(M \otimes i d) \circ(U \otimes i d \otimes S) \circ(i d \otimes \Delta \otimes i d) \circ(i d \otimes \Delta) \\
& =M \circ(M \otimes i d) \circ(V \otimes i d \otimes S) \circ(i d \otimes \Delta \otimes i d) \circ(i d \otimes \Delta) .
\end{aligned}
$$

But

$$
\begin{aligned}
& M \circ(M \otimes i d) \circ(U \otimes i d \otimes S) \circ(i d \otimes \Delta \otimes i d) \circ(i d \otimes \Delta) \\
& =M \circ(U \otimes\{M \circ(i d \otimes S) \circ \Delta\}) \circ(i d \otimes \Delta) \\
& =M \circ(U \otimes\{u \circ \epsilon\}) \circ(i d \otimes \Delta)=U .
\end{aligned}
$$

As a similar result is true with $U$ replace by $V$, it follows from (78) that $U=V$, which is an equivalent way of expressing (71).

It can shown by a similar argument that

$$
\sum_{(b)} h\left(M\left(b_{(2)} \otimes a\right)\right) S\left(b_{(1)}\right)=\sum_{(a)} h\left(M\left(b \otimes a_{(2)}\right)\right) a_{(1)}
$$

for all $a, b \in \mathcal{A}$. 


\section{THE RIGHT AND LEFT REGULAR COMODULES}

The right regular comodule of $\mathcal{A}$ is defined to have $\mathcal{A}$ itself as its carrier space, with $\Delta$ providing the coaction $\pi_{\mathcal{A}}^{R}$. That is

$$
V=\mathcal{A}, \pi_{\mathcal{A}}^{R}=\Delta
$$

In this case the conditions (43) and (44) for $\pi_{\mathcal{A}}^{R}$ to be a right coaction are immediately satisfied by virtue of the assumptions (9) and (12).

The left regular comodule of $\mathcal{A}$ is also defined to have $\mathcal{A}$ itself as its carrier space, but has $\sigma \circ(S \otimes i d) \circ \Delta$ as its coaction $\pi_{\mathcal{A}}^{L}$. That is

$$
V=\mathcal{A}, \pi_{\mathcal{A}}^{L}=\sigma \circ(S \otimes i d) \circ \Delta
$$

For this case the condition (43) for $\pi_{\mathcal{A}}^{L}$ to be a right coaction is satisfied by the assumptions (9) and (17), while the condition (44) is again satisfied as a result of the assumption (12). It should be noted that $\pi_{\mathcal{A}}^{R}$ and $\pi_{\mathcal{A}}^{L}$ are both right coactions, for, as discussed in Appendix A, the designation 'left' of $\pi_{\mathcal{A}}^{L}$ comes from its relation to the left regular action of a group in the special case in which the dual $\mathcal{A}^{\prime}$ is a group algebra. It is also useful to note that (81) implies that

$$
\Delta=\left(S^{-1} \otimes i d\right) \circ \sigma \circ \pi_{\mathcal{A}}^{L}
$$

The notation of (48) can be developed further by writing

$$
\pi_{\mathcal{A}}^{X}(a)=\sum_{[a]} a_{[1]}^{X} \otimes a_{[2]}^{X},
$$

for $X=R$ and $X=L$, where $a_{[1]}^{X}$ and $a_{[1]}^{X}$ are elements of $\mathcal{A}$. Then (20) and (80) imply that

$$
a_{[1]}^{R}=a_{(1)}, a_{[2]}^{R}=a_{(2)},
$$

but (20) and (81) give

$$
a_{[1]}^{L}=a_{(2)}, a_{[2]}^{L}=S\left(a_{(1)}\right) .
$$

The right and left regular corepresentations are both unitary, provided that the inner products on the carrier space $\mathcal{A}$ are chosen in the following way: 
1. for the right regular corepresentation take

$$
\langle a, b\rangle_{\mathcal{A}}=(a, b)^{R}=h\left(M\left(a^{*} \otimes b\right)\right) \text { for all } a, b \in \mathcal{A}
$$

2. for the left regular corepresentation take

$$
\langle a, b\rangle_{\mathcal{A}}=(a, b)^{L}=h\left(M\left(b \otimes\left(S^{2}(a)\right)^{*}\right)\right) \text { for all } a, b \in \mathcal{A} .
$$

In outline the proofs of these statements are as follows. For the right regular corepresentation, the unitary condition (51) with the choice (86) for inner product and with (84) becomes

$$
\sum_{(v)} h\left(M\left(w^{*} \otimes v_{(1)}\right)\right) S\left(v_{(2)}\right)=\sum_{(w)} h\left(M\left(w_{(1)}^{*} \otimes v\right)\right) w_{(2)}^{*},
$$

which in turn reduces to (71) with the substitutions $w=a^{*}$ and $v=b$. Similarly, for the left regular corepresentation, the unitary condition (51) with the choice (87) for inner product and with (85) becomes

$$
\sum_{(v)} h\left(M\left(v_{(2)} \otimes\left(S^{2}(w)\right)^{*}\right)\right) S^{2}\left(v_{(1)}\right)=\sum_{(w)} h\left(M\left(v \otimes\left(S^{2}\left(w_{(2)}\right)\right)^{*}\right)\right)\left(S\left(w_{(1)}\right)\right)^{*} .
$$

With the substitutions $w=S^{-1}\left(a^{*}\right)$ and $v=S^{-1}(b)$, and the application of (17), (37), and (39), this reduces again to (81).

With the choices (86) and (87), both $(a, a)^{R}$ and $(a, a)^{L}$ are real and positive for all non-zero $a \in \mathcal{A}$. For $(a, a)^{R}$ this is an immediate consequence of (61), while for $(a, a)^{L}$ the demonstration requires (63), (16), (62), and (39) as well.

The inner products (86) and (87) will be used throughout this paper. In the special case in which $\mathcal{A}$ is the space of functions defined on a compact group $\mathcal{G}$, both $(a, b)^{R}$ and $(a, b)^{L}$ reduce to the usual inner product

$$
\int_{\mathcal{G}} \overline{a(x)} b(x) \mathrm{d} x .
$$

It is worth noting at this stage that the invariance properties (64) imply that 


$$
\left(M_{C^{\prime}, \mathcal{A}} \circ(h \otimes i d) \circ \pi_{\mathcal{A}}^{R}\right)(a)=\left(M_{\mathcal{A}, C^{c}} \circ(i d \otimes h) \circ \pi_{\mathcal{A}}^{R}\right)(a)=h(a) 1_{\mathcal{A}}
$$

and

$$
\left(M_{\boldsymbol{C}^{\prime \prime}, \mathcal{A}} \circ(h \otimes i d) \circ \pi_{\mathcal{A}}^{L}\right)(a)=\left(M_{\mathcal{A}, C^{\prime}} \circ(i d \otimes h) \circ \pi_{\mathcal{A}}^{L}\right)(a)=h(a) 1_{\mathcal{A}}
$$

for all $a \in \mathcal{A}$. Acting with $h$ again in (88) and (89), and using (60), gives

$$
\left(M_{\mathbb{C}^{n}} \circ(h \otimes h) \circ \pi_{\mathcal{A}}^{X}\right)(a)=h(a)
$$

for both $X=R$ and $L$ and for all $a \in \mathcal{A}$. In terms of the notation of (83), this can be re-expressed as

$$
h(a)=\sum_{[a]} h\left(a_{[1]}^{X}\right) h\left(a_{[2]}^{X}\right)
$$

for $X=R$ and $L$ and for all $a \in \mathcal{A}$.

The effects of the right and left regular coactions on products are quite different. For the right regular coaction, (80) and (10) imply immediately that

$$
\pi_{\mathcal{A}}^{R} \circ M=(M \otimes M) \circ(i d \otimes \sigma \otimes i d) \circ\left(\pi_{\mathcal{A}}^{R} \otimes \pi_{\mathcal{A}}^{R}\right)
$$

whereas for the left regular coaction, (81), (10), and (16) show that

$$
\pi_{\mathcal{A}}^{L} \circ M=(M \otimes M) \circ(i d \otimes i d \otimes \sigma) \circ(i d \otimes \sigma \otimes i d) \circ\left(\pi_{\mathcal{A}}^{L} \otimes \pi_{\mathcal{A}}^{L}\right),
$$

which contains an extra twist factor $\sigma$.

\section{BASIS FUNCTIONS}

\section{A. Definitions and properties}

Suppose that $\pi_{j k}^{p}$ are the matrix coefficients of a corepresentation $\pi^{p}$ of $\mathcal{A}$ of finite di-

mension $d_{p}$. Then the basis functions $\psi_{j}^{p R}$ of $\pi^{p}$ with respect to the right regular coaction may be defined to be a set of $d_{p}$ elements of $\mathcal{A}$ that have the property that 


$$
\pi_{\mathcal{A}}^{R}\left(\psi_{j}^{p R}\right)=\sum_{k=1}^{d_{p}} \psi_{k}^{p R} \otimes \pi_{k j}^{p}
$$

for all $j=1,2, \ldots, d_{p}$. Similarly the basis functions $\psi_{j}^{p L}$ of $\pi^{p}$ with respect to the left regular coaction may be defined as a set of $d_{p}$ elements of $\mathcal{A}$ that have the property that

$$
\pi_{\mathcal{A}}^{L}\left(\psi_{j}^{p L}\right)=\sum_{k=1}^{d_{p}} \psi_{k}^{p L} \otimes \pi_{k j}^{p}
$$

for all $j=1,2, \ldots, d_{p}$.

In the right regular coaction case, an example of a set of basis functions is provided (for any fixed choice of $\ell=1,2, \ldots$ ) by

$$
\psi_{j}^{p R}=\pi_{\ell j}^{p}
$$

for all $j=1,2, \ldots, d_{p}$. Likewise, in the left regular coaction case, an example is provided (for any fixed choice of $\ell=1,2, \ldots$ ) by

$$
\psi_{j}^{p L}=S^{-2}\left(\pi_{j \ell}^{p *}\right)
$$

for all $j=1,2, \ldots, d_{p}$ (provided that the copresentation $\pi^{p}$ is unitary).

One very useful result, which comes from applying (95), (82), (17), (37), and (39), is that

$$
\pi_{\mathcal{A}}^{L}\left(\left(S^{2}\left(\psi_{k}^{q L}\right)\right)^{*}\right)=\sum_{t=1}^{d_{p}}\left(S^{2}\left(\psi_{t}^{q L}\right)\right)^{*} \otimes \pi_{t k}^{q *}
$$

for all $k=1,2, \ldots, d_{p}$.

In spite of the fact that the inner products (86) and (87) for the right and left regular coactions are different, in both the cases the basis functions possess the same orthogonality properties, which are as follows: If $\psi_{k}^{q X}$ and $\phi_{j}^{p X}$ are basis functions of the unitary irreducible corepresentations $\pi^{q}$ and $\pi^{p}$ of $\mathcal{A}$, then

$$
\left(\psi_{k}^{q X}, \phi_{j}^{p X}\right)^{X}=0 \text { unless } p=q \text { and } j=k,
$$

and 


$$
\left(\psi_{j}^{p X}, \phi_{j}^{p X}\right)^{X} \text { is independent of } j, \text { for } j=1,2, \ldots, d_{p} .
$$

Here $X$ denotes both $R$ and $L$, and in (100) $\psi_{j}^{p X}$ and $\phi_{j}^{p X}$ need not be identical sets. Indeed, with $X=R$, if the functions $\psi_{j}^{p R}$ and $\phi_{j}^{p R}$ are defined by

$$
\psi_{j}^{p R}=\pi_{s j}^{p}, \phi_{j}^{p R}=\pi_{t j}^{p},
$$

and with $X=L$, if the functions $\psi_{j}^{p L}$ and $\phi_{j}^{p L}$ are similarly defined by

$$
\psi_{j}^{p L}=S^{-2}\left(\pi_{j s}^{p *}\right), \phi_{j}^{p L}=S^{-2}\left(\pi_{j t}^{p *}\right),
$$

then in both cases

$$
\left(\psi_{j}^{p X}, \phi_{j}^{p X}\right)^{X}=\left(\left(\mathbf{F}^{p}\right)^{-1}\right)_{t s} / \operatorname{tr}\left(\left(\mathbf{F}^{p}\right)^{-1}\right)
$$

for $j=1,2, \ldots, d_{p}$.

The proofs of (99), (100), and (103) will now be outlined. Applying (92) and (88) to $M\left(\psi_{k}^{q R *} \otimes \phi_{j}^{p R}\right)$ gives

$$
h\left(M\left(\psi_{k}^{q R *} \otimes \phi_{j}^{p R}\right)\right) 1_{\mathcal{A}}=\sum_{s, t} h\left(M\left(\psi_{t}^{q R *} \otimes \phi_{s}^{p R}\right)\right) M\left(\pi_{t k}^{q *} \otimes \pi_{s j}^{p}\right) .
$$

A further application of $h$ to both sides gives

$$
\left(\psi_{k}^{q X}, \phi_{j}^{p X}\right)^{X}=\sum_{s, t}\left(\psi_{t}^{q X}, \phi_{s}^{p X}\right)^{X} h\left(M\left(\pi_{t k}^{q *} \otimes \pi_{s j}^{p}\right)\right)
$$

with $X=R$, where (86) and (60) have been invoked. Similarly, applying (93) and (98) to $M\left(\phi_{j}^{p L} \otimes\left(S^{2}\left(\psi_{k}^{q L}\right)\right)^{*}\right)$, and then applying (89) to the result gives

$$
h\left(M\left(\phi_{j}^{p L} \otimes\left(S^{2}\left(\psi_{k}^{q L}\right)\right)^{*}\right)\right) 1_{\mathcal{A}}=\sum_{s, t} h\left(M\left(\phi_{s}^{p L} \otimes\left(S^{2}\left(\psi_{t}^{q L}\right)\right)^{*}\right)\right) M\left(\pi_{t k}^{q *} \otimes \pi_{s j}^{p}\right) .
$$

A further application of $h$ to both sides gives (104) with $X=L$, where this time (87) and (60) have been used. Thus, in both cases, it follows from (104), (52), (65), and (68) that

$$
\left(\psi_{k}^{q X}, \phi_{j}^{p X}\right)^{X}=\sum_{s, t}\left(\psi_{t}^{q X}, \phi_{s}^{p X}\right)^{X} \delta^{q p} \delta_{k j}\left(\left(\mathbf{F}^{p}\right)^{-1}\right)_{s t} / \operatorname{tr}\left(\left(\mathbf{F}^{p}\right)^{-1}\right) .
$$

This implies that (99) is true, and if $j=k$ and $p=q$, then (105) and (99) together give 


$$
\left(\psi_{j}^{p X}, \phi_{j}^{p X}\right)^{X}=\sum_{s}\left(\psi_{s}^{p X}, \phi_{s}^{p X}\right)^{X}\left(\left(\mathbf{F}^{p}\right)^{-1}\right)_{s s} / \operatorname{tr}\left(\left(\mathbf{F}^{p}\right)^{-1}\right)
$$

As the right-hand side of (106) is independent of $j$, so too must be the left-hand side, which

thereby establishes (100). Finally the combination of (86) and (101) and the combination of (87) and (102) both produce the result

$$
\left(\psi_{j}^{p X}, \phi_{j}^{p X}\right)^{X}=h\left(M\left(S\left(\pi_{j s}^{p}\right) \otimes \pi_{t j}^{p}\right)\right)
$$

for $X=R$ and for $X=L$, which gives (103) when (68) is used.

\section{B. Projection operators}

The argument in Appendix $\mathrm{C}$ suggests the following definition. Let $\pi^{p}$ be a unitary irreducible corepresentation of $\mathcal{A}$ of dimension $d_{p}$ with matrix coefficients $\pi_{m n}^{p}$. Then the projection operators $\mathcal{P}_{m n}^{p R}$ and $\mathcal{P}_{m n}^{p L}$ are defined by

$$
\mathcal{P}_{m n}^{p X}(a)=d_{p} \sum_{[a]} a_{[1]}^{X} h\left(M\left(\pi_{m n}^{p *} \otimes a_{[2]}^{X}\right)\right.
$$

for $X=R$ and $X=L$, for all $m, n=1,2, \ldots, d_{p}$, and all $a \in \mathcal{A}$.

These projection operators have the following two useful properties: Let $\pi^{p}$ and $\pi^{q}$ be two unitary irreducible corepresentations of $\mathcal{A}$ of dimensions $d_{p}$ and $d_{q}$ with matrix coefficients $\pi_{m n}^{p}$ and $\pi_{j k}^{q}$

1. Then

$$
\mathcal{P}_{m n}^{p X} \mathcal{P}_{j k}^{q X}=d_{p} \delta^{p q}\left\{\left(\left(\mathbf{F}^{p}\right)^{-1}\right)_{n j} / \operatorname{tr}\left(\left(\mathbf{F}^{p}\right)^{-1}\right)\right\} \mathcal{P}_{m k}^{p X}
$$

for $X=R$ and $X=L$, for all $m, n=1,2, \ldots, d_{p}$, and for all $j, k=1,2, \ldots, d_{q}$.

2. Also, if $\psi_{k}^{q X}$ are basis functions for $\pi^{q}$, then

$$
\mathcal{P}_{m n}^{p X}\left(\psi_{k}^{q X}\right)=d_{p} \delta^{p q} \delta_{n k} \sum_{\ell=1}^{d_{p}} \psi_{\ell}^{q X}\left(\left(\mathbf{F}^{p}\right)^{-1}\right)_{\ell m} / \operatorname{tr}\left(\left(\mathbf{F}^{p}\right)^{-1}\right)
$$

for $X=R$ and $X=L$, for all $m, n=1,2, \ldots, d_{p}$, and for all $k=1,2, \ldots, d_{q}$. 
The proof of (108) is as follows. For any $f \in \mathcal{A}$, (107) and (9) imply that

$$
\mathcal{P}_{m n}^{p X}\left(\mathcal{P}_{j k}^{q X}(f)\right)=d_{p} d_{q} \sum_{[f]} f_{[1]}^{X} h\left(M\left(\pi_{j k}^{q *} \otimes\left(f_{[2]}^{X}\right)_{(1)}\right)\right) h\left(M\left(\pi_{m n}^{p *} \otimes\left(f_{[2]}^{X}\right)_{(2)}\right)\right) .
$$

On applying (79) with $a=f_{[2]}^{X}$ and $b=\pi_{j k}^{q *}$, this reduces

$$
\mathcal{P}_{m n}^{p X}\left(\mathcal{P}_{j k}^{q X}(f)\right)=d_{p} d_{q} \sum_{[f]} \sum_{\ell=1}^{d_{q}} f_{[1]}^{X} h\left(M\left(\pi_{\ell k}^{q *} \otimes f_{[2]}^{X}\right)\right) h\left(M\left(\pi_{m n}^{p *} \otimes S\left(\pi_{j \ell}^{q *}\right)\right),\right.
$$

so

$$
\mathcal{P}_{m n}^{p X}\left(\mathcal{P}_{j k}^{q X}(f)\right)=d_{p} \sum_{\ell=1}^{d_{q}} \mathcal{P}_{\ell k}^{q X}(f) h\left(M\left(\pi_{m n}^{p *} \otimes S\left(\pi_{j \ell}^{q *}\right)\right),\right.
$$

which, by (52), (16), and (68), gives (108).

To prove (109) it suffices to note that (107), (94) and (87) imply that

$$
\mathcal{P}_{m n}^{p X}\left(\psi_{k}^{q X}\right)=d_{p} \sum_{\ell=1}^{d_{p}} \psi_{\ell}^{q X} h\left(M\left(\pi_{m n}^{p *} \otimes \pi_{\ell k}^{q}\right)\right),
$$

which, by (68), leads immediately to (108).

\section{TENSOR PRODUCTS AND CLEBSCH-GORDAN COEFFICIENTS}

\section{A. Ordinary and twisted tensor products}

The tensor product of two corepresentations $\pi_{V}$ and $\pi_{W}$ of $\mathcal{A}$ (with carrier spaces $V$ and $W$ respectively) is the mapping $\pi_{V} \otimes \pi_{W}$ from $V \otimes W$ to $V \otimes W \otimes \mathcal{A}$ that is defined by

$$
\left(\pi_{V} \otimes \pi_{W}\right)(v \otimes w)=\left((i d \otimes i d \otimes M) \circ(i d \otimes \sigma \otimes i d) \circ\left(\pi_{V} \otimes \pi_{W}\right)\right)(v \otimes w)
$$

for all $v \in V$ and all $w \in W$. It is easily checked that the conditions (43) and (44) are satisfied with $\pi_{V}$ replaced by $\pi_{V} \otimes \pi_{W}$ and $V$ replaced by $V \otimes W$, so $\pi_{V} \otimes \pi_{W}$ is indeed a coaction of $\mathcal{A}$ with carrier space $V \otimes W$. If $V$ and $W$ are of finite dimensions $d_{V}$ and $d_{W}$, with bases $v_{1}, v_{2}, \ldots, v_{d_{V}}$, and $w_{1}, w_{2}, \ldots, w_{d_{W}}$, then (45) and (110) give

$$
\left(\pi_{V} \otimes \pi_{W}\right)\left(v_{j} \otimes w_{k}\right)=\sum_{s=1}^{d_{V}} \sum_{t=1}^{d_{W}}\left(v_{s} \otimes w_{t}\right) \otimes\left(M\left(\pi_{s j}^{V} \otimes \pi_{t k}^{W}\right)\right),
$$


which implies that the matrix coefficients of $\pi_{V} \otimes \pi_{W}$ are given by

$$
\left(\pi_{V} \otimes \pi_{W}\right)_{s t, j k}=M\left(\pi_{s j}^{V} \otimes \pi_{t k}^{W}\right)
$$

Henceforth it will always be assumed that in tensor product matrices such as $\pi_{V} \otimes \pi_{W}$ the pair of indices that specify the columns have the ordering:

$$
(j, k)=(1,1),(1,2), \ldots,\left(1, d_{W}\right),(2,1),(2,2), \ldots
$$

and that the same ordering applies to the rows.

There exists a second tensor product of $\pi_{V}$ and $\pi_{W}$ that has the same carrier space $V \otimes W$. This will be called the twisted tensor product, and is defined as the mapping $\pi_{V} \widetilde{\nabla} \pi_{W}$ from $V \otimes W$ to $V \otimes W \otimes \mathcal{A}$ that is given by

$$
\left(\pi_{V} \tilde{\otimes} \pi_{W}\right)(v \otimes w)=\left((i d \otimes i d \otimes M) \circ(i d \otimes i d \otimes \sigma) \circ(i d \otimes \sigma \otimes i d) \circ\left(\pi_{V} \otimes \pi_{W}\right)\right)(v \otimes w)
$$

for all $v \in V$ and all $w \in W$. It is again easily checked that the conditions (43) and (44) are satisfied with $\pi_{V}$ replaced by $\pi_{V} \widetilde{\otimes} \pi_{W}$ and $V$ replaced by $V \otimes W$, so $\pi_{V} \widetilde{\otimes} \pi_{W}$ is also a coaction of $\mathcal{A}$ with carrier space $V \otimes W$. Then (45) and (114) give

$$
\left(\pi_{V} \widetilde{\otimes} \pi_{W}\right)\left(v_{j} \otimes w_{k}\right)=\sum_{s=1}^{d_{V}} \sum_{t=1}^{d_{W}}\left(v_{s} \otimes w_{t}\right) \otimes\left(M\left(\pi_{t k}^{W} \otimes \pi_{s j}^{V}\right)\right),
$$

which implies that the matrix coefficients of $\pi_{V} \widetilde{\otimes} \pi_{W}$ are given by

$$
\left(\pi_{V} \tilde{\otimes} \pi_{W}\right)_{s t, j k}=M\left(\pi_{t k}^{W} \otimes \pi_{s j}^{V}\right)
$$

The tensor product $\pi_{W} \otimes \pi_{V}$, whose carrier space is $\pi_{W} \otimes \pi_{V}$, has matrix coefficients that are given (according to (112) ) by

$$
\left(\pi_{W} \otimes \pi_{V}\right)_{t s, k j}=M\left(\pi_{t k}^{W} \otimes \pi_{s j}^{V}\right)
$$

As the matrix coefficients of ([16) and (117) differ only in their ordering of the pairs of indices that label their rows (and, in the corresponding manner, their columns), it follows 
that $\pi_{V} \widetilde{\otimes} \pi_{W}$ and $\pi_{W} \otimes \pi_{V}$ are equivalent corepresentations. If $\mathcal{A}$ is coquasitriangular (that is, if $\mathcal{A}^{\prime}$ is quasitriangular (c.f Drinfel'd $\mathrm{d}^{30}$, then $\pi_{V} \otimes \pi_{W}$ and $\pi_{W} \otimes \pi_{V}$ are equivalent, so in this case $\pi_{V} \otimes \pi_{W}$ and $\pi_{V} \widetilde{\otimes} \pi_{W}$ are equivalent. (Of course, in the special case in which $\mathcal{A}$ is commutative, the corepresentations $\pi_{V} \otimes \pi_{W}$ and $\pi_{V} \widetilde{\otimes} \pi_{W}$ are identical).

Applying these considerations to the special case in which $\pi_{V}=\pi^{p}$ and $\pi_{W}=\pi^{q}$ are two irreducible unitary corepresentations of $\mathcal{A}$, (112) and (116) become

$$
\left(\pi^{p} \otimes \pi^{q}\right)_{s t, j k}=M\left(\pi_{s j}^{p} \otimes \pi_{t k}^{q}\right)
$$

and

$$
\left(\pi^{p} \widetilde{\otimes} \pi^{q}\right)_{s t, j k}=M\left(\pi_{t k}^{q} \otimes \pi_{s j}^{p}\right)
$$

respectively.

\section{B. Characters}

The character of a corepresentation $\pi_{V}$ of $\mathcal{A}$ of dimension $d_{V}$ is defined in terms of its matrix coefficients by

$$
\chi^{V}=\sum_{j=1}^{d_{V}} \pi_{j j}^{V},
$$

so $\chi^{V}$ is also an element of $\mathcal{A}$. Clearly equivalent corepresentations have identical characters.

If $\pi^{p}$ and $\pi^{q}$ are two irreducible unitary corepresentations of $\mathcal{A}$ (assumed to be inequivalent if $p \neq q$ ) and if $\chi^{p}$ and $\chi^{q}$ are their corresponding characters, then (67) and (68) imply that

$$
h\left(M\left(\chi^{p *} \otimes \chi^{q}\right)\right)=h\left(M\left(\chi^{q} \otimes \chi^{p *}\right)\right)=\delta^{p q} .
$$

If $\pi_{V}$ is a (completely) reducible corepresentation of $\mathcal{A}$ that is equivalent to the direct sum $\sum \oplus n^{p} \pi^{p}$, then the number of times $n^{p}$ that the irreducible corepresentation $\pi^{p}$ (or a corepresentation equivalent to $\pi^{p}$ ) appears in the reduction of $\pi_{V}$ is given by 


$$
\chi^{V}=\sum_{p} n^{p} \chi^{p}
$$

so, by (121),

$$
n^{p}=h\left(M\left(\chi^{V} \otimes \chi^{p *}\right)\right)=h\left(M\left(\chi^{p *} \otimes \chi^{V}\right)\right)
$$

For the tensor product $\pi^{p} \otimes \pi^{q}$ of two irreducible unitary corepresentations $\pi^{p}$ and $\pi^{q}$ the character is just $\chi^{p} \chi^{q}\left(=M\left(\chi^{p} \otimes \chi^{q}\right)\right)$, so the number of times $n_{p q}^{r}$ that the irreducible corepresentation $\pi^{r}$ (or a corepresentation equivalent to $\pi^{r}$ ) appears in the reduction of $\pi^{p} \otimes \pi^{q}$ is given by

$$
n_{p q}^{r}=h\left(\chi^{p} \chi^{q} \chi^{r *}\right)=h\left(\chi^{r *} \chi^{p} \chi^{q}\right)
$$

If $\bar{\pi}^{p}$ is the irreducible unitary corepresentation that is conjugate to $\pi^{p}$, then (59) and (62) show that

$$
n_{p q}^{r}=n_{\bar{p} r}^{q}, n_{r \bar{p}}^{q}=n_{q p}^{r}
$$

where $n_{\bar{p} r}^{q}$ is the number of times that $\pi^{q}$ (or its equivalent) appears in the reduction of $\bar{\pi}^{p} \otimes \pi^{r}$, and $n_{r \bar{p}}^{q}$ is the number of times that $\pi^{q}$ (or its equivalent) appears in the reduction of $\pi^{r} \otimes \bar{\pi}^{p}$.

\section{Clebsch-Gordan coefficients}

As above, suppose that the the tensor product $\pi^{p} \otimes \pi^{q}$ of two irreducible unitary corepresentations $\pi^{p}$ and $\pi^{q}$ is reducible, and that $n_{p q}^{r}$ is the number of times that the irreducible corepresentation $\pi^{r}$ (or a corepresentation equivalent to it) appears in the reduction of $\pi^{p} \otimes \pi^{q}$. If $\pi^{p}$ has carrier space $V^{p}$ with basis elements $v_{1}^{p}, v_{1}^{p}, \ldots, v_{d_{p}}^{p}$ and $\pi^{q}$ has carrier

space $V^{q}$ with basis elements $v_{1}^{q}, v_{1}^{q}, \ldots, v_{d_{q}}^{q}$, then the set of elements $v_{j}^{p} \otimes v_{k}^{q}$ form a basis for $V^{p} \otimes V^{q}$, the carrier space of $\pi^{p} \otimes \pi^{q}$, and consequently appropriate linear combinations form bases for all the irreducible corepresentations $\pi^{r}$ that appear in the reduction of the tensor product. Let $w_{\ell}^{r, \alpha}$ be such a combination, so that 


$$
w_{\ell}^{r, \alpha}=\sum_{j=1}^{d_{p}} \sum_{k=1}^{d_{q}}\left(\begin{array}{cc|c}
p & q & r, \alpha \\
j & k & \ell
\end{array}\right) v_{j}^{p} \otimes v_{k}^{q}
$$

for $\ell=1,2, \ldots, d_{r}$, and $\alpha=1,2, \ldots, n_{p q}^{r}$, and

$$
\left(\pi^{p} \otimes \pi^{q}\right)\left(w_{\ell}^{r, \alpha}\right)=\sum_{u=1}^{d_{r}} w_{u}^{r, \alpha} \otimes \pi_{u l}^{r},
$$

for $u=1,2, \ldots, d_{r}$, and $\alpha=1,2, \ldots, n_{p q}^{r}$. The inverse of (126) is

$$
v_{j}^{p} \otimes v_{k}^{q}=\sum_{r} \sum_{\alpha=1}^{n_{p q}^{r}} \sum_{\ell=1}^{d_{r}}\left(\begin{array}{ll|ll}
r, & \alpha & p & q \\
\ell & & j & k
\end{array}\right) w_{\ell}^{r, \alpha},
$$

for $j=1,2, \ldots, d_{p}$ and $k=1,2, \ldots, d_{q}$. The Clebsch-Gordan coefficients defined in (126) form the elements of a $d_{p} \times d_{q}$ matrix $\mathbf{C}$, while the inverse coefficients defined in (128) form the elements of $\mathbf{C}^{-1}$, where

$$
\mathbf{C}^{-1}\left(\pi^{p} \otimes \pi^{q}\right) \mathbf{C}=\sum_{r} \oplus n_{p q}^{r} \pi^{r}
$$

That is,

$$
\sum_{j=1}^{d_{p}} \sum_{t=1}^{d_{q}}\left(\pi^{p} \otimes \pi^{q}\right)_{i s, j t}\left(\begin{array}{cc|cc}
p & q & r & \alpha \\
j & t & \ell
\end{array}\right)=\sum_{u=1}^{d_{r}}\left(\begin{array}{cc|cc}
p & q & r & , \alpha \\
i & s & u
\end{array}\right) \pi_{u \ell}^{r}
$$

for $i=1,2, \ldots, d_{p}, s=1,2, \ldots, d_{q}, \ell=1,2, \ldots, d_{r}$, and $\alpha=1,2, \ldots, n_{p q}^{r}$.

\section{Products of basis functions and Clebsch-Gordan coefficients}

Consider first the right regular corepresentation. If $\phi_{j}^{p R}$ and $\psi_{k}^{q R}$ are basis functions of the unitary irreducible corepresentations $\pi^{p}$ and $\pi^{q}$ of $\mathcal{A}$, then (92) and (118) imply that

$$
\pi_{\mathcal{A}}^{R}\left(M\left(\phi_{j}^{p R} \otimes \psi_{k}^{q R}\right)\right)=\sum_{s=1}^{d_{p}} \sum_{t=1}^{d_{q}} M\left(\phi_{s}^{p R} \otimes \psi_{t}^{q R}\right) \otimes\left(\pi^{p} \otimes \pi^{q}\right)_{s t, j k},
$$

so that if the set of products $M\left(\phi_{j}^{p R} \otimes \psi_{k}^{q R}\right)$ (for $j=1,2, \ldots, d_{p}$, and $k=1,2, \ldots, d_{q}$ ) form a linearly independent set, then they form a basis for the tensor product corepresentation 
$\pi^{p} \otimes \pi^{q}$. Thus, by $(126)$ and $(\overline{127})$, there exists a set of elements $\theta_{\ell}^{r, \alpha R}$, all members of $\mathcal{A}$, that are defined by

$$
\theta_{\ell}^{r, \alpha R}=\sum_{j=1}^{d_{p}} \sum_{k=1}^{d_{q}}\left(\begin{array}{cc|cc}
p & q & r & , \alpha \\
j & k & \ell
\end{array}\right) M\left(\phi_{j}^{p R} \otimes \psi_{k}^{q R}\right),
$$

for $\ell=1,2, \ldots, d_{r}$, and $\alpha=1,2, \ldots, n_{p q}^{r}$, and which have the property that

$$
\pi_{\mathcal{A}}^{R}\left(\theta_{\ell}^{r, \alpha R}\right)=\sum_{u=1}^{d_{r}} \theta_{u}^{r, \alpha R} \otimes \pi_{u l}^{r}
$$

for $u=1,2, \ldots, d_{r}$, and $\alpha=1,2, \ldots, n_{p q}^{r}$. By (128), the inverse of (132) is then

$$
M\left(\phi_{j}^{p R} \otimes \psi_{k}^{q R}\right)=\sum_{r} \sum_{\alpha=1}^{n_{p q}^{r}} \sum_{\ell=1}^{d_{r}}\left(\begin{array}{ll|ll}
r, & \alpha & p & q \\
\ell & j & k
\end{array}\right) \theta_{\ell}^{r, \alpha R},
$$

for $j=1,2, \ldots, d_{p}$ and $k=1,2, \ldots, d_{q}$. On applying the projection operator $\mathcal{P}_{u l}^{r R}$ of (107) to $M\left(\phi_{j}^{p R} \otimes \psi_{k}^{q R}\right)$, and using (109), (134) and (132), it follows that

$$
\begin{aligned}
& \mathcal{P}_{u l}^{r R}\left(M\left(\phi_{j}^{p R} \otimes \psi_{k}^{q R}\right)\right) \\
& =\sum_{\alpha=1}^{n_{p q}^{r}} \sum_{v=1}^{d_{r}} \sum_{s=1}^{d_{p}} \sum_{t=1}^{d_{q}}\left(\begin{array}{ll|ll}
r & \alpha & p & q \\
\ell & j & k
\end{array}\right)\left(\begin{array}{ll|ll}
p & q & r & \alpha \\
s & t & v
\end{array}\right) \\
& \times d_{r}\left\{\left(\left(\mathbf{F}^{r}\right)^{-1}\right)_{v u} / \operatorname{tr}\left(\left(\mathbf{F}^{r}\right)^{-1}\right)\right\} M\left(\phi_{s}^{p R} \otimes \psi_{t}^{q R}\right)
\end{aligned}
$$

for $j=1,2, \ldots, d_{p}$ and $k=1,2, \ldots, d_{q}$. However, the definition (107) taken in conjunction with (131) and (118) gives

$$
\mathcal{P}_{u l}^{r R}\left(M\left(\phi_{j}^{p R} \otimes \psi_{k}^{q R}\right)\right)=d_{r} \sum_{s=1}^{d_{p}} \sum_{t=1}^{d_{q}} M\left(\phi_{s}^{p R} \otimes \psi_{t}^{q R}\right) h\left(\pi_{u l}^{r *} \pi_{s j}^{p} \pi_{t k}^{q}\right),
$$

so equating coefficients of $M\left(\phi_{s}^{p R} \otimes \psi_{t}^{q R}\right)$ in (135) and (136) yields

$$
\begin{aligned}
h\left(\pi_{u l}^{r *} \pi_{s j}^{p} \pi_{t k}^{q}\right)= & \sum_{\alpha=1}^{n_{p q}^{r}} \sum_{v=1}^{d_{r}}\left(\begin{array}{ll|ll}
r & \alpha & p & q \\
\ell & j & k
\end{array}\right)\left(\begin{array}{ll|l}
p & q & r \\
s & t & v
\end{array}\right) \\
& \times\left\{\left(\left(\mathbf{F}^{r}\right)^{-1}\right)_{v u} / \operatorname{tr}\left(\left(\mathbf{F}^{r}\right)^{-1}\right)\right\}
\end{aligned}
$$

for all $j=1,2, \ldots, d_{p}, k=1,2, \ldots, d_{q}$, and $l=1,2, \ldots, d_{r}$. Of course, this implies that 


$$
\begin{aligned}
h\left(\pi_{u l}^{r *} \pi_{t k}^{q} \pi_{s j}^{p}\right)= & \sum_{\alpha=1}^{n_{q p}^{r}} \sum_{v=1}^{d_{r}}\left(\begin{array}{ll|ll}
r & \alpha & q & p \\
\ell & k & j
\end{array}\right)\left(\begin{array}{ll|ll}
q & p & r & , \alpha \\
t & s & v
\end{array}\right) \\
& \times\left\{\left(\left(\mathbf{F}^{r}\right)^{-1}\right)_{v u} / \operatorname{tr}\left(\left(\mathbf{F}^{r}\right)^{-1}\right)\right\}
\end{aligned}
$$

for all $j=1,2, \ldots, d_{p}, k=1,2, \ldots, d_{q}$, and $l=1,2, \ldots, d_{r}$.

Although the conclusions (137) and (138) also follow from consideration of the left regular coaction, some of the intermediate results are significantly different in this case. Firstly (93) implies that

$$
\pi_{\mathcal{A}}^{L}\left(M\left(\phi_{j}^{p L} \otimes \psi_{k}^{q L}\right)\right)=\sum_{s=1}^{d_{p}} \sum_{t=1}^{d_{q}} M\left(\phi_{s}^{p L} \otimes \psi_{t}^{q L}\right) \otimes M\left(\pi_{t k}^{q} \otimes \pi_{s j}^{p}\right),
$$

so, by (119),

$$
\pi_{\mathcal{A}}^{L}\left(M\left(\phi_{j}^{p L} \otimes \psi_{k}^{q L}\right)\right)=\sum_{s=1}^{d_{p}} \sum_{t=1}^{d_{q}} M\left(\phi_{s}^{p L} \otimes \psi_{t}^{q L}\right) \otimes\left(\pi^{p} \widetilde{\otimes} \pi^{q}\right)_{s t, j k},
$$

so that if the set of products $M\left(\phi_{j}^{p L} \otimes \psi_{k}^{q L}\right)$ (for $j=1,2, \ldots, d_{p}$, and $\left.k=1,2, \ldots, d_{q}\right)$ form a linearly independent set, then they form a basis for the twisted tensor product corepresentation $\pi^{p} \widetilde{\otimes} \pi^{q}$. However, on writing $\Phi_{k j}^{q p}=M\left(\phi_{j}^{p L} \otimes \psi_{k}^{q L}\right)$, (140) can be re-expressed as

$$
\pi_{\mathcal{A}}^{L}\left(\Phi_{k j}^{q p}\right)=\sum_{s=1}^{d_{p}} \sum_{t=1}^{d_{q}} \Phi_{t s}^{q p} \otimes\left(\pi^{q} \otimes \pi^{p}\right)_{t s, k j}
$$

That is, the set $\Phi_{k j}^{q p}$ (for $k=1,2, \ldots, d_{q}$ and $j=1,2, \ldots, d_{p}$ ) form a basis for the ordinary tensor product corepresentation $\pi^{q} \otimes \pi^{p}$. Consequently, there exists a set of elements $\theta_{\ell}^{r, \alpha L}$, all members of $\mathcal{A}$, that are defined by

$$
\theta_{\ell}^{r, \alpha L}=\sum_{j=1}^{d_{p}} \sum_{k=1}^{d_{q}}\left(\begin{array}{ll|ll}
q & p & r & , \alpha \\
k & j & \ell
\end{array}\right) \Phi_{k j}^{q p}
$$

for $\ell=1,2, \ldots, d_{r}$, and $\alpha=1,2, \ldots, n_{q p}^{r}$, and which have the property that

$$
\pi_{\mathcal{A}}^{R}\left(\theta_{\ell}^{r, \alpha L}\right)=\sum_{u=1}^{d_{r}} \theta_{u}^{r, \alpha L} \otimes \pi_{u l}^{r}
$$

for $u=1,2, \ldots, d_{r}$, and $\alpha=1,2, \ldots, n_{q p}^{r}$. Thus 


$$
\theta_{\ell}^{r, \alpha L}=\sum_{j=1}^{d_{p}} \sum_{k=1}^{d_{q}}\left(\begin{array}{ll|ll}
q & p & r & , \alpha \\
k & j & \ell
\end{array}\right) M\left(\phi_{j}^{p L} \otimes \psi_{k}^{q L}\right)
$$

whose inverse is

$$
M\left(\phi_{j}^{p L} \otimes \psi_{k}^{q L}\right)=\sum_{r} \sum_{\alpha=1}^{n_{q p}^{r}} \sum_{\ell=1}^{d_{r}}\left(\begin{array}{ll|ll}
r & \alpha & q & p \\
\ell & & k & j
\end{array}\right) \theta_{\ell}^{r, \alpha L}
$$

for $j=1,2, \ldots, d_{p}$ and $k=1,2, \ldots, d_{q}$. Applying the projection operator $\mathcal{P}_{u l}^{r L}$ of (107) to $M\left(\phi_{j}^{p L} \otimes \psi_{k}^{q L}\right)$, and using (109), (145) and (144), it follows that

$$
\begin{aligned}
& \mathcal{P}_{u l}^{r L}\left(M\left(\phi_{j}^{p L} \otimes \psi_{k}^{q L}\right)\right) \\
& =\sum_{\alpha=1}^{n_{q p}^{r}} \sum_{v=1}^{d_{r}} \sum_{s=1}^{d_{p}} \sum_{t=1}^{d_{q}}\left(\begin{array}{lll|ll}
r & \alpha & q & p \\
\ell & k & j
\end{array}\right)\left(\begin{array}{ll|ll}
q & p & r & , \\
t & s & v
\end{array}\right) \\
& \times d_{r}\left\{\left(\left(\mathbf{F}^{r}\right)^{-1}\right)_{v u} / \operatorname{tr}\left(\left(\mathbf{F}^{r}\right)^{-1}\right)\right\} M\left(\phi_{s}^{p L} \otimes \psi_{t}^{q L}\right)
\end{aligned}
$$

for $j=1,2, \ldots, d_{p}$ and $k=1,2, \ldots, d_{q}$. However, the definition (107) taken in conjunction with (139) gives

$$
\mathcal{P}_{u l}^{r L}\left(M\left(\phi_{j}^{p L} \otimes \psi_{k}^{q L}\right)\right)=d_{r} \sum_{s=1}^{d_{p}} \sum_{t=1}^{d_{q}} M\left(\phi_{s}^{p L} \otimes \psi_{t}^{q L}\right) h\left(\pi_{u l}^{r *} \pi_{t k}^{q} \pi_{s j}^{p}\right),
$$

so equating coefficients of $M\left(\phi_{s}^{p L} \otimes \psi_{t}^{q L}\right)$ in (146) and (147) yields (138) again.

It is also possible to obtain (137) (and hence (138)) without making any linear independence assumptions about the set of products $M\left(\phi_{j}^{p R} \otimes \psi_{k}^{q R}\right)$, for by (111), (126), (127), and (128),

$$
\begin{aligned}
& \sum_{r} \sum_{\alpha=1}^{n_{p q}^{r}} \sum_{s=1}^{d_{p}} \sum_{t=1}^{d_{q}} \sum_{v, w=1}^{d_{r}}\left(\begin{array}{cc|cc}
r & , \alpha & p & q \\
w & & j & k
\end{array}\right)\left(\begin{array}{cc|cc}
p & q & r & , \alpha \\
s & t & v
\end{array}\right) v_{s}^{p} \otimes v_{t}^{q} \otimes \pi_{v w}^{r} \\
& =\sum_{s=1}^{d_{p}} \sum_{t=1}^{d_{q}} v_{s}^{p} \otimes v_{t}^{q} \otimes M\left(\pi_{s j}^{p} \otimes \pi_{t k}^{q}\right)
\end{aligned}
$$

for all $j=1,2, \ldots, d_{p}$, and $k=1,2, \ldots, d_{q}$. As the set $v_{s}^{p} \otimes v_{t}^{q}$ (for $s=1,2, \ldots, d_{p}$, and $\left.t=1,2, \ldots, d_{q}\right)$ is certainly linearly independent, it follows that

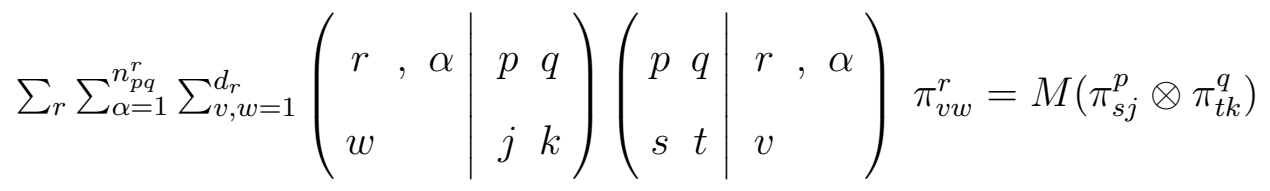


(for $j, s=1,2, \ldots, d_{p}$, and $k, t=1,2, \ldots, d_{q}$ ). On replacing $r$ by $r^{\prime}$ in the sums of on the left-hand side, multiplying through by $\pi_{u \ell}^{r *}$ from the left, applying the Haar functional $h$, and using (65) and (68), one regains (137).

\section{THE IRREDUCIBLE TENSOR OPERATORS}

\section{A. Introduction}

Let $\pi^{q}$ be a unitary irreducible right coaction of $\mathcal{A}$ of dimension $d_{q}$ with matrix coefficients $\pi_{j k}^{q}$. It will be shown that within both the right and the left regular corepresentation formalisms there exist two types of irreducible tensor operators that both belong to this

corepresentation $\pi^{q}$. These will be denoted by $Q_{j}^{q X}$ and $\widetilde{Q}_{j}^{q X}$ (for $j=1,2, \ldots, d_{q}$ and for $X=R$ or $L$ ), and will be called ordinary and twisted irreducible tensor operators respectively. Naturally the two types of irreducible tensor operators coincide in the special case in which $\mathcal{A}$ is commutative. Moreover, it should be noted that if $\mathcal{A}^{\text {op }}$ is the Hopf algebra in which the multiplication operator $M$ and antipode $S$ of $\mathcal{A}$ are replaced by $M \circ \sigma$ and $S^{-1}$ respectively, then the 'twisted' irreducible tensor operators for $\mathcal{A}$ are the 'ordinary' irreducible tensor operators for $\mathcal{A}^{\text {op }}$ and the 'ordinary' irreducible tensor operators for $\mathcal{A}$ are the 'twisted' irreducible tensor operators for $\mathcal{A}^{o p}$.

\section{B. Definitions in the right regular corepresentation formalism}

1. Definition of the ordinary irreducible tensor operators $Q_{j}^{q R}$

The ordinary irreducible tensor operators $Q_{j}^{q R}$ belonging to the unitary irreducible right coaction $\pi^{q}$ of $\mathcal{A}$ are defined to be members of $\mathcal{L}(\mathcal{A})$ that satisfy the condition

$$
\left((i d \otimes M) \circ(\Delta \otimes i d) \circ\left(Q_{j}^{q R} \otimes S\right) \circ \Delta\right)(a)=\sum_{k=1}^{d_{q}} Q_{k}^{q R}(a) \otimes \pi_{k j}^{q}
$$

for all $a \in \mathcal{A}$ and all $j=1,2, \ldots, d_{q}$. Hereafter $\mathcal{L}(\mathcal{A})$ denotes the set of linear operators that $\operatorname{map} \mathcal{A}$ into $\mathcal{A}$. 
Clearly this definition involves only quantities defined on $\mathcal{A}$, for the right-hand side is a member of $\mathcal{A} \otimes \mathcal{A}$. By virtues of (80) this definition can be written equivalently in terms of the right regular coaction $\pi_{\mathcal{A}}^{R}$ as

$$
\left((i d \otimes M) \circ\left(\pi_{\mathcal{A}}^{R} \otimes i d\right) \circ\left(Q_{j}^{q R} \otimes S\right) \circ \pi_{\mathcal{A}}^{R}\right)(a)=\sum_{k=1}^{d_{q}} Q_{k}^{q R}(a) \otimes \pi_{k j}^{q}
$$

for all $a \in \mathcal{A}$ and all $j=1,2, \ldots, d_{q}$. (The motivation behind the definition (148) is explained in Appendix B.2.a).

It will now be shown that (148) provides a consistent definition, in that it can be reexpressed by saying that the operators $Q_{j}^{q R}$ (for $j=1,2, \ldots, d_{q}$ ) form the basis of a carrier space for a certain right coaction of $\mathcal{A}$. The motivation for this definition is given in Appendix B.2.b., where the special case in which $\mathcal{A}$ is finite-dimensional is considered in detail, but for general case it is necessary to apply additional conditions to the domain of this coaction. The analysis of Appendix B.2.b implies that if $\mathcal{A}$ is finite-dimensional then, for every operator $Q \in \mathcal{L}(\mathcal{A})$, there exist operators $Q_{i} \in \mathcal{L}(\mathcal{A})$ and elements $q_{i}$, both indexed by the same finite index set $I$, such that

$$
\sum_{i \in I} Q_{i}(a) \otimes q_{i}=((i d \otimes M) \circ(\Delta \otimes i d) \circ(Q \otimes S) \circ \Delta)(a)
$$

for all $a \in \mathcal{A}$, but this is not necessarily true for every $Q \in \mathcal{L}(\mathcal{A})$ if $\mathcal{A}$ is infinite-dimensional. However, one can always define a subspace $\mathcal{T}(\mathcal{A})$ of $\mathcal{L}(\mathcal{A})$ by the requirement that $Q \in \mathcal{T}(\mathcal{A})$ if (i) $Q \in \mathcal{L}(\mathcal{A})$, (ii) $Q$ satisfies (150) with $I$ finite, and (iii) each $Q_{i}$ appearing on the left-hand side of (150) also satisfies a condition of the same form. This subspace $\mathcal{T}(\mathcal{A})$ is certainly not empty, for the identity operator belongs to it (c.f. (170)), as does every irreducible tensor operator $Q_{j}^{q R}$ (c.f.(148)), and, as just noted, if $\mathcal{A}$ is finite-dimensional then $\mathcal{T}(\mathcal{A})$ is identical to $\mathcal{L}(\mathcal{A})$. The definition of the required right coaction, which will be denoted by $\pi_{\mathcal{T}(\mathcal{A})}^{R}$, is then that it is the mapping of $\mathcal{T}(\mathcal{A})$ into $\mathcal{T}(\mathcal{A}) \otimes \mathcal{A}$ that given by

$$
\pi_{\mathcal{T}(\mathcal{A})}^{R}(Q)=\sum_{[Q]} Q_{[1]} \otimes Q_{[2]}
$$

where $Q_{[1]} \in \mathcal{T}(\mathcal{A})$ and $Q_{[1]} \in \mathcal{A}$ are such that 


$$
\sum_{[Q]} Q_{[1]}(a) \otimes Q_{[2]}=((i d \otimes M) \circ(\Delta \otimes i d) \circ(Q \otimes S) \circ \Delta)(a)
$$

for all $Q \in \mathcal{T}(\mathcal{A})$ and all $a \in \mathcal{A}$. It is then quite easily shown (using the identities (8) to (19)) that $\pi_{\mathcal{T}(\mathcal{A})}^{R}$ satisfies (43) and (44) (with $\pi_{V}$ and $V$ replaced by $\pi_{\mathcal{T}(\mathcal{A})}^{R}$ and $\mathcal{T}(\mathcal{A})$ respectively, and with all operators of $\mathcal{T}(\mathcal{A})$ acting on any member of $\mathcal{A}$ according to the prescription of (152)). That is,

$$
\sum_{[Q]} \sum_{\left[Q_{[1]}\right]}\left(Q_{[1]}\right)_{[1]}(a) \otimes\left(Q_{[1]}\right)_{[2]} \otimes Q_{[2]}=\sum_{[Q]} Q_{[1]}(a) \otimes \Delta\left(Q_{[2]}\right)
$$

and

$$
\sum_{[Q]}\left(Q_{[1]}\right)(a) \epsilon\left(Q_{[2]}\right)=Q(a)
$$

for all $Q \in \mathcal{T}(\mathcal{A})$ and all $a \in \mathcal{A}$. Hence $\pi_{\mathcal{T}(\mathcal{A})}^{R}$ is indeed a right coaction with carrier space $\mathcal{T}(\mathcal{A})$. Thus (151) and (152) imply that the definition (148) can be written equivalently as

$$
\pi_{\mathcal{T}(\mathcal{A})}^{R}\left(Q_{j}^{q R}\right)=\sum_{k=1}^{d_{q}} Q_{k}^{q R} \otimes \pi_{k j}^{q}
$$

(for all $j=1,2, \ldots$ ). Because (154) is similar in form to (45), and as $\pi_{\mathcal{T}(\mathcal{A})}^{R}$ is a right coaction with carrier space $\mathcal{T}(\mathcal{A})$, the consistency of the definition (148) is now ensured.

\section{Definition of the twisted irreducible tensor operators $\widetilde{Q}_{j}^{q R}$}

The twisted irreducible tensor operators $\widetilde{Q}_{j}^{q R}$ belonging to the unitary irreducible right coaction $\pi^{q}$ of $\mathcal{A}$ are defined to be members of $\mathcal{L}(\mathcal{A})$ that satisfy the condition

$$
\left((i d \otimes M) \circ(i d \otimes \sigma) \circ(\Delta \otimes i d) \circ\left(\widetilde{Q}_{j}^{q R} \otimes S^{-1}\right) \circ \Delta\right)(a)=\sum_{k=1}^{d_{q}} \widetilde{Q}_{k}^{q R}(a) \otimes \pi_{k j}^{q}
$$

for all $a \in \mathcal{A}$ and all $j=1,2, \ldots, d_{q}$. This can be written equivalently in terms of the right regular coaction $\pi_{\mathcal{A}}^{R}$ as

$$
\left((i d \otimes M) \circ(i d \otimes \sigma) \circ\left(\pi_{\mathcal{A}}^{R} \otimes i d\right) \circ\left(\widetilde{Q}_{j}^{q R} \otimes S^{-1}\right) \circ \pi_{\mathcal{A}}^{R}\right)(a)=\sum_{k=1}^{d_{q}} \widetilde{Q}_{k}^{q R}(a) \otimes \pi_{k j}^{q}
$$


for all $a \in \mathcal{A}$ and all $j=1,2, \ldots, d_{q}$. Both (155) and (156) differ from the corresponding definitions (148) and (149) only in the replacement of $M$ by $M \circ \sigma$ and $S$ by $S^{-1}$ (neither of which have any effect in the special case in which $\mathcal{A}$ is commutative). (See Appendix B.2.a for further discussion of this pair of substitutions. It should be recorded that Rittenberg and Scheunert ${ }^{8}$ noted previously, in the context of what was essentially the 'abstract carrier space formalism' (form (3) of Section I) as generalized to irreducible representations of $\mathcal{A}^{\prime}$, that these substitutions do produce another type of irreducible tensor operator, but they did not pursue this observation at all.)

The demonstration that (155) provides a consistent definition again involves showing that it can be re-expressed by saying that the operators $\widetilde{Q}_{j}^{q R}$ (for $j=1,2, \ldots, d_{q}$ ) form the basis of a carrier space for a right coaction of $\mathcal{A}$. This right coaction $\tilde{\pi}_{\mathcal{T}(\mathcal{A})}^{R}$ (and its associated space $\mathcal{T}(\mathcal{A}))$ are essentially obtained from $\pi_{\mathcal{T}(\mathcal{A})}^{R}$ by replacing $M$ by $M \circ \sigma$ and $S$ by $S^{-1}$, so $\tilde{\pi}_{\mathcal{T}(\mathcal{A})}^{R}$ is defined as the mapping of $\mathcal{T}(\mathcal{A})$ into $\mathcal{T}(\mathcal{A}) \otimes \mathcal{A}$ that given by

$$
\tilde{\pi}_{\mathcal{T}(\mathcal{A})}^{R}(Q)=\sum_{[Q]} Q_{[1]} \otimes Q_{[2]}
$$

where $Q_{[1]} \in \mathcal{T}(\mathcal{A})$ and $Q_{[1]} \in \mathcal{A}$ are such that

$$
\sum_{[Q]} Q_{[1]}(a) \otimes Q_{[2]}=\left((i d \otimes(M \circ \sigma)) \circ(\Delta \otimes i d) \circ\left(Q \otimes S^{-1}\right) \circ \Delta\right)(a)
$$

for all $Q \in \mathcal{T}(\mathcal{A})$ and all $a \in \mathcal{A}$. Then (157) and (158) imply that the definition (155) can be written equivalently as

$$
\tilde{\pi}_{\mathcal{T}(\mathcal{A})}^{R}\left(\widetilde{Q}_{j}^{q R}\right)=\sum_{k=1}^{d_{q}} \widetilde{Q}_{k}^{q R} \otimes \pi_{k j}^{q}
$$

(for all $j=1,2, \ldots$ ), which then ensures the consistency of the definition (155).

\section{Definitions in the left regular corepresentation formalism}

\section{Definition of the ordinary irreducible tensor operators $Q_{j}^{q L}$}

The ordinary irreducible tensor operators $Q_{j}^{q L}$ belonging to the unitary irreducible right coaction $\pi^{q}$ of $\mathcal{A}$ are defined to be members of $\mathcal{L}(\mathcal{A})$ that satisfy the condition 


$$
\left((i d \otimes M) \circ\left(\pi_{\mathcal{A}}^{L} \otimes i d\right) \circ\left(Q_{j}^{q L} \otimes S\right) \circ \pi_{\mathcal{A}}^{L}\right)(a)=\sum_{k=1}^{d_{q}} Q_{k}^{q L}(a) \otimes \pi_{k j}^{q}
$$

for all $a \in \mathcal{A}$ and all $j=1,2, \ldots, d_{q}$. (This is can be obtained from (149) by replacing $X=R$ by $X=L$, the justification being discussed in more detail in Appendix B.3.a). In terms of the elementary operations of $\mathcal{A}$, (160) can be re-expressed using (81) as

$$
\left(\sigma \circ(S \otimes i d) \circ(M \otimes i d) \circ(i d \otimes \Delta) \circ\left(S \otimes Q_{j}^{q L}\right) \circ \Delta\right)(a)=\sum_{k=1}^{d_{q}} Q_{k}^{q L}(a) \otimes \pi_{k j}^{q}
$$

for all $a \in \mathcal{A}$ and all $j=1,2, \ldots, d_{q}$.

The demonstration that (160) provides a consistent definition proceeds in the same way as above. In this case the appropriate right coaction will be denoted by $\pi_{\mathcal{T}(\mathcal{A})}^{L}$, and will be defined as the mapping of $\mathcal{T}(\mathcal{A})$ into $\mathcal{T}(\mathcal{A}) \otimes \mathcal{A}$ that given by

$$
\pi_{\mathcal{T}(\mathcal{A})}^{L}(Q)=\sum_{[Q]} Q_{[1]} \otimes Q_{[2]}
$$

where $Q_{[1]} \in \mathcal{T}(\mathcal{A})$ and $Q_{[1]} \in \mathcal{A}$ are such that

$$
\sum_{[Q]} Q_{[1]}(a) \otimes Q_{[2]}=(\sigma \circ(S \otimes i d) \circ(M \otimes i d) \circ(i d \otimes \Delta) \circ(S \otimes Q) \circ \Delta)(a)
$$

for all $Q \in \mathcal{T}(\mathcal{A})$ and all $a \in \mathcal{A}$. (Here the subspace $\mathcal{T}(\mathcal{A})$ of $\mathcal{L}(\mathcal{A})$ is defined as in Section VI.B.1, but with the right-hand side of (163) replacing the right-hand side of (152) in (150). The motivation for this definition is given in Appendix B.3.b). Then the definition (160) can be written equivalently as

$$
\pi_{\mathcal{T}(\mathcal{A})}^{L}\left(Q_{j}^{q L}\right)=\sum_{k=1}^{d_{q}} Q_{k}^{q L} \otimes \pi_{k j}^{q}
$$

(for all $j=1,2, \ldots$ ), which then guarantees its consistency.

\section{Definition of the twisted irreducible tensor operators $\widetilde{Q}_{j}^{q L}$}

The twisted irreducible tensor operators $\widetilde{Q}_{j}^{q L}$ belonging $\pi^{q}$ of $\mathcal{A}$ are defined to be members of $\mathcal{L}(\mathcal{A})$ that satisfy the condition 


$$
\left((i d \otimes M) \circ(i d \otimes \sigma) \circ\left(\pi_{\mathcal{A}}^{L} \otimes i d\right) \circ\left(\widetilde{Q}_{j}^{q L} \otimes S^{-1}\right) \circ \pi_{\mathcal{A}}^{L}\right)(a)=\sum_{k=1}^{d_{q}} \widetilde{Q}_{k}^{q L}(a) \otimes \pi_{k j}^{q}
$$

for all $a \in \mathcal{A}$ and all $j=1,2, \ldots, d_{q}$. In terms of the elementary operations of $\mathcal{A}$, (165) can be re-expressed using (81) as

$$
\left((i d \otimes M) \circ(\sigma \otimes S) \circ(i d \otimes \sigma) \circ(i d \otimes \Delta) \circ\left(i d \otimes \widetilde{Q}_{j}^{q L}\right) \circ \Delta\right)(a)=\sum_{k=1}^{d_{q}} \widetilde{Q}_{k}^{q L}(a) \otimes \pi_{k j}^{q}
$$

for all $a \in \mathcal{A}$ and all $j=1,2, \ldots, d_{q}$. The definition (165) differs from the corresponding definition (160) only in the replacement of $M$ by $M \circ \sigma$ and $S$ by $S^{-1}$ (neither of which have any effect in the special case in which $\mathcal{A}$ is commutative). However, because the two $S$ factors in (161) have different origins, these substitutions do not convert (161) into (166). (See Appendix B.3.a for a further discussion of this point).

The consistency of the definition (165) is again shown in the same way as above. In this case the appropriate right coaction will be denoted by $\widetilde{\pi}_{\mathcal{T}(\mathcal{A})}^{L}$, and will be defined as the mapping of $\mathcal{T}(\mathcal{A})$ into $\mathcal{T}(\mathcal{A}) \otimes \mathcal{A}$ that given by

$$
\tilde{\pi}_{\mathcal{T}(\mathcal{A})}^{L}(Q)=\sum_{[Q]} Q_{[1]} \otimes Q_{[2]}
$$

where $Q_{[1]} \in \mathcal{T}(\mathcal{A})$ and $Q_{[1]} \in \mathcal{A}$ are such that

$$
\sum_{[Q]} Q_{[1]}(a) \otimes Q_{[2]}=((i d \otimes M) \circ(\sigma \otimes S) \circ(i d \otimes \sigma) \circ(i d \otimes \Delta) \circ(i d \otimes Q) \circ \Delta)(a)
$$

for all $Q \in \mathcal{T}(\mathcal{A})$ and all $a \in \mathcal{A}$. (Here the subspace $\mathcal{T}(\mathcal{A})$ of $\mathcal{L}(\mathcal{A})$ is defined as in Section VI.B.1, but with the right-hand side of (168) replacing the right-hand side of (152) in $(\mathbf{1 5 0})$. The motivation for this definition is given in Appendix B.3.b). Then the definition (165) can be written equivalently as

$$
\widetilde{\pi}_{\mathcal{T}(\mathcal{A})}^{L}\left(\widetilde{Q}_{j}^{q L}\right)=\sum_{k=1}^{d_{q}} \widetilde{Q}_{k}^{q L} \otimes \pi_{k j}^{q}
$$

(for all $j=1,2, \ldots$ ), which then implies the consistency of (165). 


\section{The identity operator as an irreducible tensor operator}

Suppose that $Q$ is the identity operator id of $\mathcal{L}(\mathcal{A})$ (so that $Q(a)=a$ for all $a \in \mathcal{A}$ ). Then, on using (9) and (18), it follows that

$$
(i d \otimes M) \circ(\Delta \otimes i d) \circ(i d \otimes S) \circ \Delta=i d \otimes 1_{\mathcal{A}},
$$

which, by (148), leads to the agreeable conclusion that the identity operator $i d$ is an ordinary irreducible tensor operator in the right regular corepresentation formalism for the one-dimensional identity corepresentation whose sole matrix coefficient is $1_{\mathcal{A}}$.

It is easily checked (using (155), (161), and (166) in place of (148)), that $i d$ is also a twisted irreducible tensor operator for the identity corepresentation in the right regular corepresentation formalism, as well as being a both an ordinary and a twisted irreducible tensor operator for the identity corepresentation in the left regular corepresentation formalism.

In fact, if one were to adopt the view that these results concerning the identity operator are an essential requirement of any sensible definition of irreducible tensor operators, the fact that they are not true if $M$ is replaced by $M \circ \sigma$ but $S$ is left unchanged, nor if $M$ is left unchanged but $S$ is replaced by $S^{-1}$, then precludes further consideration of these possibilities.

\section{E. Products as irreducible tensor operators}

Suppose now that $\psi_{j}^{q R}$ and $\psi_{j}^{q L}$ are sets of basis functions for $\pi^{q}$ (as defined in (94) and (95) respectively) and that the operators $Q_{j}^{q X}$ and $\widetilde{Q}_{j}^{q X}$ are defined by

$$
\left.\begin{array}{l}
Q_{j}^{q R}(a)=M\left(\psi_{j}^{q R} \otimes a\right), \\
\widetilde{Q}_{j}^{q R}(a)=M\left(a \otimes \psi_{j}^{q R}\right), \\
Q_{j}^{q L}(a)=M\left(a \otimes \psi_{j}^{q L}\right), \\
\widetilde{Q}_{j}^{q L}(a)=M\left(\psi_{j}^{q L} \otimes a\right),
\end{array}\right\}
$$


for all $a \in \mathcal{A}$. Then the identities (21) to (32) imply that the operators $Q_{j}^{q R}, \widetilde{Q}_{j}^{q R}, Q_{j}^{q L}$, and $\widetilde{Q}_{j}^{q L}$ do indeed satisfy (148), (155), (161), and (166) respectively, and so are irreducible tensor operators belonging to $\pi^{q}$.

\section{F. Two useful identities for the ordinary irreducible tensor operators $Q_{j}^{q X}$ and $\widetilde{Q}_{j}^{q X}$}

If $Q_{k}^{q X}$ is an ordinary irreducible tensor operator belonging to the unitary irreducible right coaction $\pi^{q}$ of $\mathcal{A}$ and $\psi_{j}^{p X}$ is a set of basis functions for the unitary irreducible right coaction $\pi^{p}$ of $\mathcal{A}$, then

$$
\pi_{\mathcal{A}}^{X}\left(Q_{k}^{q X}\left(\psi_{j}^{p X}\right)\right)=\sum_{s=1}^{d_{p}} \sum_{t=1}^{d_{q}}\left(Q_{t}^{q X}\left(\psi_{s}^{p X}\right)\right) \otimes\left(M\left(\pi_{t k}^{q} \otimes \pi_{s j}^{p}\right)\right),
$$

for $X=R$ and $L$, for all $j=1,2, \ldots, d_{p}$, and $k=1,2, \ldots, d_{q}$. That is, by (118),

$$
\pi_{\mathcal{A}}^{X}\left(Q_{k}^{q X}\left(\psi_{j}^{p X}\right)\right)=\sum_{s=1}^{d_{p}} \sum_{t=1}^{d_{q}}\left(Q_{t}^{q X}\left(\psi_{s}^{p X}\right)\right) \otimes\left(\pi^{q} \otimes \pi^{p}\right)_{t s, k j},
$$

for $X=R$ and $L$, for all $j=1,2, \ldots, d_{p}$ and $k=1,2, \ldots, d_{q}$.

By contrast, if $\widetilde{Q}_{k}^{q X}$ is a twisted irreducible tensor operator belonging $\pi^{q}$, then

$$
\pi_{\mathcal{A}}^{X}\left(\widetilde{Q}_{k}^{q X}\left(\psi_{j}^{p X}\right)\right)=\sum_{s=1}^{d_{p}} \sum_{t=1}^{d_{q}}\left(\widetilde{Q}_{t}^{q X}\left(\psi_{s}^{p X}\right)\right) \otimes\left(M\left(\pi_{s j}^{p} \otimes \pi_{t k}^{q}\right)\right)
$$

for $X=R$ and $L$, for all $j=1,2, \ldots, d_{p}$ and $k=1,2, \ldots, d_{q}$. It should be noted that the factors in the second term of the right-hand side of (174) are interchanged relative to those of (172), which implies, by (119), that

$$
\pi_{\mathcal{A}}^{X}\left(\widetilde{Q}_{k}^{q X}\left(\psi_{j}^{p X}\right)\right)=\sum_{s=1}^{d_{p}} \sum_{t=1}^{d_{q}}\left(\widetilde{Q}_{t}^{q X}\left(\psi_{s}^{p X}\right)\right) \otimes\left(\pi^{q} \widetilde{\otimes} \pi^{p}\right)_{t s, k j},
$$

for $X=R$ and $L$, for all $j=1,2, \ldots, d_{p}$, and $k=1,2, \ldots, d_{q}$, which involves the twisted tensor product.

The proof of (172) is as follows. Applying (94) or (95) to the case $a=\psi_{u}^{p X}$ and invoking (149) or (160) (as appropriate) gives

$$
\sum_{v=1}^{d_{p}}(i d \otimes M)\left(\pi_{\mathcal{A}}^{X}\left(Q_{j}^{q X}\left(\psi_{v}^{p X}\right)\right) \otimes S\left(\pi_{v u}^{p}\right)\right)=\sum_{k=1}^{d_{q}}\left(Q_{k}^{q X}\left(\psi_{u}^{p X}\right)\right) \otimes \pi_{k j}^{q} .
$$


However, for any $a \in \mathcal{A}$

$$
(i d \otimes M)\left(\pi_{\mathcal{A}}^{X}(a) \otimes S\left(\pi_{v u}^{p}\right)\right)=\sum_{[a]} a_{[1]} \otimes M\left(a_{[2]} \otimes S\left(\pi_{v u}^{p}\right)\right),
$$

the right-hand side of which, on multiplication from the right with $1_{\mathcal{A}} \otimes \pi_{u i}^{p}$, and summing

over $u$, and using (52) and (53), reduces to $\delta_{i v} \pi_{\mathcal{A}}^{X}(a)$. The desired result (172) is then obtained by multiplying both sides of (176) from the right with $1_{\mathcal{A}} \otimes \pi_{u i}^{p}$ and summing over $u$. The line of proof for (174) is similar.

\section{G. Products of operators}

If $Q$ and $Q^{\prime}$ are any two members of $\mathcal{T}(\mathcal{A})$ and $\pi_{\mathcal{T}(\mathcal{A})}^{X}$ is the ordinary right coaction defined in (151) and (152) (for $X=R$ ) and (162) and (163) (for $X=L$ ), then

$$
\pi_{\mathcal{T}(\mathcal{A})}^{X}\left(Q Q^{\prime}\right)=\pi_{\mathcal{T}(\mathcal{A})}^{X}(Q) \pi_{\mathcal{T}(\mathcal{A})}^{X}\left(Q^{\prime}\right)
$$

for $X=R$ and $L$. This can be re- expressed as

$$
\left(\pi_{\mathcal{T}(\mathcal{A})}^{X} \circ \widehat{M}\right)\left(Q \otimes Q^{\prime}\right)=\left((\widehat{M} \otimes M) \circ(i d \otimes \sigma \otimes i d) \circ\left(\pi_{\mathcal{T}(\mathcal{A})}^{X} \otimes \pi_{\mathcal{T}(\mathcal{A})}^{X}\right)\right)\left(Q \otimes Q^{\prime}\right)
$$

for $X=R$ and $L$, where the operator multiplication operation $\widehat{M}$ is defined by

$$
\widehat{M}\left(Q \otimes Q^{\prime}\right)=Q \circ Q^{\prime}
$$

for all $Q, Q^{\prime} \in \mathcal{T}(\mathcal{A})$.

By contrast, if $\tilde{\pi}_{\mathcal{T}(\mathcal{A})}^{X}$ is the twisted right coaction defined in (157) and (158) (for $X=R$ ) and (167) and (168) (for $X=L)$, then

$$
\begin{aligned}
& \left(\widetilde{\pi}_{\mathcal{T}(\mathcal{A})}^{X} \circ \widehat{M}\right)\left(Q \otimes Q^{\prime}\right)= \\
& \left((\widehat{M} \otimes M) \circ(i d \otimes i d \otimes \sigma) \circ(i d \otimes \sigma \otimes i d) \circ\left(\widetilde{\pi}_{\mathcal{T}(\mathcal{A})}^{X} \otimes \widetilde{\pi}_{\mathcal{T}(\mathcal{A})}^{X}\right)\right)\left(Q \otimes Q^{\prime}\right)
\end{aligned}
$$

for $X=R$ and $L$, whose right-hand side involves an extra twist factor $(i d \otimes i d \otimes \sigma)$ relative to the corresponding result (178).

The proofs of these statements just involve a straightforward application of the identities (8) to (19). 


\section{THEOREMS OF THE WIGNER-ECKART TYPE}

If $\pi^{p}, \pi^{q}$, and $\pi^{r}$ are unitary irreducible corepresentations of $\mathcal{A}$ of dimensions $d_{p}, d_{q}$, and $d_{r}$ respectively, $\phi_{j}^{p X}$ and $\psi_{\ell}^{r X}$ are basis functions belonging to $\pi^{p}$ and $\pi^{r}$, and $Q_{k}^{q X}$ is an ordinary irreducible tensor operator belonging to $\pi^{q}$, then

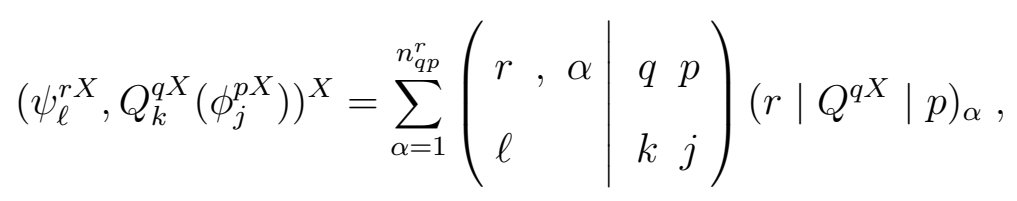

for $X=R$ and $L$, all $j=1,2, \ldots, d_{p}$, all $k=1,2, \ldots, d_{q}$, and all $\ell=1,2, \ldots, d_{r}$. Here the reduced matrix elements $\left(r\left|Q^{q X}\right| p\right)_{\alpha}$ are given by

$$
\begin{aligned}
\left(r\left|Q^{q X}\right| p\right)_{\alpha} & = \\
& \sum_{s=1}^{d_{p}} \sum_{t=1}^{d_{q}} \sum_{u, v=1}^{d_{r}}\left(\psi_{u}^{r X}, Q_{t}^{q X}\left(\phi_{s}^{p X}\right)\right)^{X}\left(\begin{array}{ll|l}
q & p & r, \alpha \\
t & s & v
\end{array}\right) \\
& \times\left\{\left(\left(\mathbf{F}^{r}\right)^{-1}\right)_{v u} / \operatorname{tr}\left(\left(\mathbf{F}^{r}\right)^{-1}\right)\right\}
\end{aligned}
$$

for $\alpha=1,2, \ldots, n_{q p}^{r}$, and the inner products $(,)^{R}$ and $(,)^{L}$ are defined in (86) and (87).

On the other hand, if $\widetilde{Q}_{k}^{q X}$ is a twisted irreducible tensor operator belonging to $\pi^{q}$, then

$$
\left(\psi_{\ell}^{r X}, \widetilde{Q}_{k}^{q X}\left(\phi_{j}^{p X}\right)\right)^{X}=\sum_{\alpha=1}^{n_{p q}^{r}}\left(\begin{array}{ll|ll}
r, \alpha & p & q \\
\ell & j & k
\end{array}\right)\left(r\left|\widetilde{Q}^{q X}\right| p\right)_{\alpha},
$$

for $X=R$ and $L$, all $j=1,2, \ldots, d_{p}$, all $k=1,2, \ldots, d_{q}$, and all $\ell=1,2, \ldots, d_{r}$, where the reduced matrix elements $\left(r\left|\widetilde{Q}^{q X}\right| p\right)_{\alpha}$ are given by

$$
\begin{aligned}
\left(r\left|\widetilde{Q}^{q X}\right| p\right)_{\alpha} & = \\
& \sum_{s=1}^{d_{p}} \sum_{t=1}^{d_{q}} \sum_{u, v=1}^{d_{r}}\left(\psi_{u}^{r X}, \widetilde{Q}_{t}^{q X}\left(\phi_{s}^{p X}\right)\right)^{X}\left(\begin{array}{ll|l}
p & q & r, \alpha \\
s & t & v
\end{array}\right) \\
& \times\left\{\left(\left(\mathbf{F}^{r}\right)^{-1}\right)_{v u} / \operatorname{tr}\left(\left(\mathbf{F}^{r}\right)^{-1}\right)\right\}
\end{aligned}
$$

for $\alpha=1,2, \ldots, n_{p q}^{r}$.

The results (181) and (183) exhibit the classic Wigner-Eckart theorem behaviour, in that they show that the $j, k$, and $\ell$ dependences of the inner products $\left(\psi_{\ell}^{r X}, Q_{k}^{q X} \phi_{j}^{p X}\right)^{X}$ and 
$\left(\psi_{\ell}^{r X}, \widetilde{Q}_{k}^{q X} \phi_{j}^{p X}\right)^{X}$ are determined only by Clebsch-Gordan coefficients, but it should be noted that in the general case in which $\mathcal{A}$ is non-commutative, the inner products for the ordinary and twisted irreducible tensor operators involve different sets of Clebsch-Gordan coefficients.

The proof of (181) in the case $X=R$ is as follows. By (92) and (172)

$$
\pi_{\mathcal{A}}^{R}\left(\psi_{\ell}^{r R *} Q_{k}^{q R}\left(\phi_{j}^{p R}\right)\right)=\sum_{s=1}^{d_{p}} \sum_{t=1}^{d_{q}} \sum_{u=1}^{d_{r}}\left(\psi_{u}^{r R *} Q_{t}^{q R}\left(\phi_{s}^{p R}\right)\right) \otimes\left(\pi_{u \ell}^{r *} \pi_{t k}^{q} \pi_{s j}^{p}\right)
$$

for all $j=1,2, \ldots, d_{p}, k=1,2, \ldots, d_{q}$, and $\ell=1,2, \ldots, d_{r}$. Then, by (91)

$$
h\left(\psi_{\ell}^{r R *} Q_{k}^{q R}\left(\phi_{j}^{p R}\right)\right)=\sum_{s=1}^{d_{p}} \sum_{t=1}^{d_{q}} \sum_{u=1}^{d_{r}} h\left(\psi_{u}^{r R *} Q_{t}^{q R}\left(\phi_{s}^{p R}\right)\right) h\left(\pi_{u \ell}^{r *} \pi_{t k}^{q} \pi_{s j}^{p}\right)
$$

for all $j=1,2, \ldots, d_{p}, k=1,2, \ldots, d_{q}$, and $\ell=1,2, \ldots, d_{r}$. Invoking (86) and (138) then immediately gives (181) and (182).

The proof of (181) in the case $X=L$ is similar. By (172), (93), and (98)

$$
\pi_{\mathcal{A}}^{L}\left(Q_{k}^{q L}\left(\phi_{j}^{p L}\right)\left(S^{2}\left(\psi_{\ell}^{r L}\right)\right)^{*}\right)=\sum_{s=1}^{d_{p}} \sum_{t=1}^{d_{q}} \sum_{u=1}^{d_{r}}\left(Q_{t}^{q L}\left(\phi_{s}^{p L}\right)\left(S^{2}\left(\psi_{u}^{r L}\right)\right)^{*}\right) \otimes\left(\pi_{u \ell}^{r *} \pi_{t k}^{q} \pi_{s j}^{p}\right)
$$

for all $j=1,2, \ldots, d_{p}, k=1,2, \ldots, d_{q}$, and $\ell=1,2, \ldots, d_{r}$. On applying (91), (87), and (138), the results (181) and (182) are obtained for this case as well.

The proof of (183) follows the same lines, but employs (174) and (137) in place of (172) and (138).

\section{PRODUCTS OF IRREDUCIBLE TENSOR OPERATORS}

If $\pi^{p}$ and $\pi^{q}$ are unitary irreducible corepresentations of $\mathcal{A}$ of dimensions $d_{p}$ and $d_{q}$ respectively, and $Q_{j}^{p X}$ and $Q_{k}^{q X}$ are ordinary irreducible tensor operators belonging to $\pi^{p}$ and $\pi^{q}$, then

$$
\pi_{\mathcal{T}(\mathcal{A})}^{X}\left(Q_{j}^{p X} Q_{k}^{q X}\right)=\sum_{s=1}^{d_{p}} \sum_{t=1}^{d_{q}}\left(Q_{s}^{p X} Q_{t}^{q X}\right) \otimes\left(\pi^{p} \otimes \pi^{q}\right)_{s t, j k}
$$

for $X=R$ and $L$ and for all $j=1,2, \ldots, d_{p}$, and $k=1,2, \ldots, d_{q}$. Here the coactions $\pi_{\mathcal{T}(\mathcal{A})}^{R}$ and $\pi_{\mathcal{T}(\mathcal{A})}^{L}$ are as defined in (151), (152), (162), and (163), and the matrix coefficients of the 
tensor product $\pi^{p} \otimes \pi^{q}$ are given in (118). (The proof of (185) just involves applying (178), (154), (164), and (179).)

Because of the similarity in form between (185) and (131), it follows immediately that

$$
Q_{\ell}^{r X, \alpha}=\sum_{j=1}^{d_{p}} \sum_{k=1}^{d_{q}}\left(\begin{array}{ll|l}
p & q & r \\
j & k & \ell
\end{array}\right) Q_{j}^{p X} Q_{k}^{q X},
$$

for $\ell=1,2, \ldots, d_{r}$, and $\alpha=1,2, \ldots, n_{p q}^{r}$. Here $Q_{\ell}^{r X, \alpha}\left(\right.$ for $\left.\alpha=1,2, \ldots, n_{p q}^{r}\right)$ are $n_{p q}^{r}$ ordinary irreducible tensor operators belonging to $\pi^{r}$ that are, in general, all different.

By contrast, if $\widetilde{Q}_{j}^{p X}$ and $\widetilde{Q}_{k}^{q X}$ are twisted irreducible tensor operators belonging to $\pi^{p}$ and $\pi^{q}$, then

$$
\widetilde{\pi}_{\mathcal{T}(\mathcal{A})}^{X}\left(\widetilde{Q}_{j}^{p X} \widetilde{Q}_{k}^{q X}\right)=\sum_{s=1}^{d_{p}} \sum_{t=1}^{d_{q}}\left(\widetilde{Q}_{s}^{p X} \widetilde{Q}_{t}^{q X}\right) \otimes\left(\pi^{p} \widetilde{\otimes} \pi^{q}\right)_{s t, j k}
$$

for $X=R$ and $L$ and for all $j=1,2, \ldots, d_{p}$, and $k=1,2, \ldots, d_{q}$. Here the right coactions $\tilde{\pi}_{\mathcal{T}(\mathcal{A})}^{R}$ and $\tilde{\pi}_{\mathcal{T}(\mathcal{A})}^{L}$ are as defined in (157), (158), (167), and (168), and the matrix coefficients of the twisted tensor product $\pi^{p} \widetilde{\otimes} \pi^{q}$ are given in (119). (This result (187) is proved using (180), (159), (169), and (179).)

The analogue of (186) for the twisted case is

$$
\widetilde{Q}_{\ell}^{r X, \alpha}=\sum_{j=1}^{d_{p}} \sum_{k=1}^{d_{q}}\left(\begin{array}{ll|l}
q & p & r, \alpha \\
k & j & \ell
\end{array}\right) \widetilde{Q}_{j}^{p X} \widetilde{Q}_{k}^{q X}
$$

for $\ell=1,2, \ldots, d_{r}$, and $\alpha=1,2, \ldots, n_{q p}^{r}$. Here $\widetilde{Q}_{\ell}^{r X, \alpha}\left(\right.$ for $\left.\alpha=1,2, \ldots, n_{q p}^{r}\right)$ are $n_{q p}^{r}$ twisted irreducible tensor operators belonging to $\pi^{r}$ that are, in general, all different.

\section{GENERALIZATION TO QUANTUM HOMOGENEOUS SPACES}

\section{A. Quantum homogeneous spaces}

The definition and role of quantum homogeneous spaces are best introduced by considering the situation first in the very well established and familiar context of a compact Lie group $\mathcal{G}$. The homogeneous space formalism for $\mathcal{G}$ has two essential features. Firstly, it is 
equivalent to the theory in which $\mathcal{G}$ acts as a transformation group on an external manifold $\mathcal{M}$, and, secondly, it is closely related to the regular representation formalisms. Both of these aspects were reviewed briefly in Section I.

With $\mathcal{G}$ taken to be a group of tranformations that act on an external manifold $\mathcal{M}$, select some typical point of $\mathcal{M}$. Let $\mathcal{H}$ be the isotropy subgroup of $\mathcal{G}$, which consists of all transformations of $\mathcal{G}$ that send this point into itself, and let $\mathcal{M}_{0}$ be the orbit of points of $\mathcal{M}$ that are obtained by acting on this typical point with every transformation of $\mathcal{G}$. In the case in which $\mathcal{G}$ is the rotation group about $O$ and $\mathcal{M}$ is $\Re^{3}$, let $\mathbf{r}_{0}$ of $\Re^{3}$ be this typical point. Then $\mathcal{H}$ is the subgroup of all rotations about the axis from $O$ to the point $\mathbf{r}_{0}$, and $\mathcal{M}_{0}$ is the sphere centred on $O$ that contains the point $\mathbf{r}_{0}$. With an appropriate choice of $\mathbf{r}_{0}, \mathcal{M}_{0}$ can be parametrised by the spherical polar coordinates $\theta$ and $\phi$. Effectively it is only the functional dependence on $\theta$ and $\phi$ that comes into symmetry arguments, the dependence on the radial distance $r$ being immaterial. That is, only the subspace $\mathcal{M}_{0}$ is actually relevant in the group theoretical calculations. However, it is easily demonstrated that there is a one-to-one correspondence between the points of $\mathcal{M}_{0}$ and the set of left cosets $T \mathcal{H}$ of $\mathcal{G}$ with respect to $\mathcal{H}$. Thus the quantities of interest are the subset $\mathcal{B}$ of $R(\mathcal{G})$ that consists of those members of $R(\mathcal{G})$ which are constant on each left coset $T \mathcal{H}$. Then the operators acting on the members of $\mathcal{B}$ that correspond to the operators $P(T)$ of (2) may be identified with the left regular operators $\widehat{L}(T)$ of $(\mathrm{A} 18)$, as restricted to act only on $\mathcal{B}$. Moreover the only part of the integral (5) that is relevant to symmetry arguments is the part involving $\theta$ and $\phi$, which is an integral over $\mathcal{M}_{0}$, and hence is equivalent to the Haar integral of (6) applied to the functions of $\mathcal{B}$. Finally, in the homogeneous space version, the irreducible tensor operators of (丑) become mappings of $\mathcal{B}$ into $\mathcal{B}$.

Henceforth the $\star$-Hopf algebra $R(\mathcal{G})$ will be denoted by $\mathcal{A}$. Then $\mathcal{B}$ becomes a $\star-$ subalgebra of $\mathcal{A}$ and a left coideal of $\mathcal{A}$. (The convention adopted here is that $\mathcal{B}$ is said to be a left coideal of $\mathcal{A}$ if $\Delta(f) \in \mathcal{A} \otimes \mathcal{B}$ for all $f \in \mathcal{B}$, and $\mathcal{B}$ is said to be a right coideal of $\mathcal{A}$ if $\Delta(f) \in \mathcal{B} \otimes \mathcal{A}$ for all $f \in \mathcal{B})$. It is also trivially true that $\mathcal{B}$ is $S^{2}$-invariant.

There also exists a parallel version of this theory associated with the right regular rep- 
resentation of $\mathcal{G}$, the operators $\widehat{R}(T)$ of which are defined by $\widehat{R}(T) f\left(T^{\prime}\right)=f\left(T^{\prime} T\right)$ for all $f$ and for all $T, T^{\prime} \in \mathcal{G}$. Then, for example, if $\mathcal{G}$ is the group of all rotations in this space about $O$ and $\mathcal{M}$ is $\Re^{3}$, in place of the transformations of (1) one could define another set in which

$$
\left(\mathbf{r}^{\prime}\right)^{T}=\mathbf{r}^{T} \mathbf{R}(T)
$$

where $\mathbf{r}^{T}$ denotes the transpose of $\mathbf{r}$. Then, for a typical point $\mathbf{r}_{0}$, there is a one-to-one correspondence between the points of the orbit $\mathcal{M}_{0}$ of $\mathbf{r}_{0}$ and the set of right cosets $\mathcal{H} T$ of $\mathcal{G}$ with respect to the corresponding isotropy subgroup $\mathcal{H}$ of $\mathbf{r}_{0}$. In this case the quantity of interest is the set $\mathcal{B}$ of representative functions of $\mathcal{G}$ which are constant on each right coset $\mathcal{H} T$. Then $\mathcal{B}$ is a $\star$-subalgebra of $\mathcal{A}$ and a right coideal of $\mathcal{A}$, and the analogues of operators $P(T)$ of (国) are the $\widehat{R}(T)$ restricted to $\mathcal{B}$. It is again trivially true that $\mathcal{B}$ is $S^{2}$-invariant.

There are various ways in which these ideas can be generalized to produce quantum homogeneous spaces ${ }^{23-26,31-36}$, but the present development follows the work of Dijkhuizen and Koornwinder ${ }^{23-26}$. In this formulation one works with a $\star$ - Hopf algebra $\mathcal{A}$ (which is in general both non-commutative and non-cocommutative), and with a $\star$-subalgebra $\mathcal{B}$ of $\mathcal{A}$ that is either a right coideal of $\mathcal{A}$ or is a left coideal of $\mathcal{A}$. (The explicit discussion in Refs. 23-26 is actually given for the former situation, but clearly the formulation can also be reexpressed for the latter situation). Dijkhuizen and Koornwinder ${ }^{23-26}$ have discussed various other algebraic objects that are associated with $\mathcal{B}$, and have shown that in the case of the quantum SU(2) group there exists a one-parameter family of such spaces (called 'quantum 2-spheres') which are mutually non-isomorphic, and they have related these to the work of Podleśs ${ }^{31}$.

For the case in which $\mathcal{B}$ is a left coideal of $\mathcal{A}$ it will be assumed, for reasons that will become clear in due course, that $\mathcal{B}$ is $S^{2}$-invariant. However, when $\mathcal{B}$ is a right coideal of $\mathcal{A}$ there is no need to make this assumption when investigating the irreducible tensor operators. Whether this assumption is needed in this case for other purposes is a matter that has been discussed by Dijkhuizen and Koornwinder ${ }^{23-24}$. 


\section{B. The restricted right and left regular coactions}

In the special case in which $\mathcal{A}$ is the dual of a group algebra, the operations of $\mathcal{A}$ corresponding to the right and left regular actions of the group algebra are the right and left regular coactions of $\mathcal{A}$. Consequently the restrictions of right and left regular actions of the group algebra to $\mathcal{B}$ correspond to the right and left regular coactions of $\mathcal{A}$ restricted to $\mathcal{B}$. These are not only the relevant operations of the classical homogeneous space formulation but they are also the basic operations of the quantum homogeneous space formulation.

Explicitly, the right regular coaction $\pi_{\mathcal{A}}^{R}$ and the left regular coaction $\pi_{\mathcal{A}}^{L}$ for a general compact quantum group algebra $\mathcal{A}$ are defined (in (43) and (81) by

$$
\pi_{\mathcal{A}}^{R}=\Delta, \pi_{\mathcal{A}}^{L}=\sigma \circ(S \otimes i d) \circ \Delta
$$

Both are right coactions of $\mathcal{A}$ with carrier space $\mathcal{A}$. The corresponding restricted right regular coaction $\pi_{\mathcal{B}}^{R}$ and restricted left regular coaction $\pi_{\mathcal{B}}^{L}$ may then be defined by

$$
\pi_{\mathcal{B}}^{R}=\pi_{\mathcal{A} \mid \mathcal{B}}^{R}=\Delta_{\mid \mathcal{B}}, \pi_{\mathcal{B}}^{L}=\pi_{\mathcal{A} \mid \mathcal{B}}^{L}=\sigma \circ(S \otimes i d) \circ \Delta_{\mid \mathcal{B}}
$$

In the context of the restricted right regular coaction it is being assumed that $\mathcal{B}$ is a right coideal of $\mathcal{A}$, whereas in the restricted left regular coaction context $\mathcal{B}$ is assumed to be a

left coideal of $\mathcal{A}$. Because of the extra twist factor $\sigma$ in the definition of $\pi_{\mathcal{B}}^{L}$, it follows that both $\pi_{\mathcal{B}}^{R}$ and $\pi_{\mathcal{B}}^{L}$ are right coactions of $\mathcal{A}$ with carrier space $\mathcal{B}$. (The role of $\pi_{\mathcal{B}}^{R}$ as a transitive $\star$-coaction corresponding to the transitive action of the quantum group associated with $\mathcal{A}$ on the quantum homogeneous space associated with $\mathcal{B}$ has been described by Dijkhuizen and Koornwinder $\left.{ }^{23,24}\right)$.

The restriction of the Haar functional $h$ of $\mathcal{A}$ to $\mathcal{B}$ provides a positive definite integral for $\mathcal{B}$ with the invariance properties

$$
\left(M_{\boldsymbol{C}, \mathcal{A}} \circ(h \otimes i d) \circ \pi_{\mathcal{B}}^{X}\right)(b)=\left(M_{\mathcal{A}, \boldsymbol{C}} \circ(i d \otimes h) \circ \pi_{\mathcal{B}}^{X}\right)(b)=h(b) 1_{\mathcal{A}}
$$

for all $b \in \mathcal{B}$ and for both $X=R$ and $X=L$ (c.f. (88) and (89)). 
The restricted right and left regular corepresentations are both unitary, provided that the inner products on the carrier space $\mathcal{B}$ are chosen in the following way:

1. for the restricted right regular corepresentation take

$$
\left\langle b, b^{\prime}\right\rangle_{\mathcal{B}}=\left(b, b^{\prime}\right)^{R}=h\left(M\left(b^{\star} \otimes b^{\prime}\right)\right) \text { for all } b, b^{\prime} \in \mathcal{B} ;
$$

2. for the restricted left regular corepresentation take

$$
\left\langle b, b^{\prime}\right\rangle_{\mathcal{B}}=\left(b, b^{\prime}\right)^{L}=h\left(M\left(b \otimes\left(S^{2}\left(b^{\prime}\right)\right)^{\star}\right)\right) \text { for all } b, b^{\prime} \in \mathcal{B} .
$$

It should be noted that $\left(S^{2}\left(b^{\prime}\right)\right)^{\star} \in \mathcal{B}$ if $\mathcal{B}$ is $S^{2}$-invariant. (The proofs of these unitary properties are essentially the same as for the corresponding results (86) and (87) for the unrestricted regular coactions).

The effects of the restricted right and left regular coactions on products are also essentially the same as for the unrestricted coactions (c.f.(92) and (93)).

\section{Basis functions for the restricted right and left regular coactions}

Suppose that $\pi_{j k}^{p}$ are the matrix coefficients of a corepresentation $\pi^{p}$ of $\mathcal{A}$ of finite dimension $d_{p}$. Then the basis functions $\psi_{j}^{p R}$ of $\pi^{p}$ with respect to the restricted right regular coaction may be defined to be a set of $d_{p}$ elements of $\mathcal{B}$ that have the property that

$$
\pi_{\mathcal{B}}^{R}\left(\psi_{j}^{p R}\right)=\sum_{k=1}^{d_{p}} \psi_{k}^{p R} \otimes \pi_{k j}^{p}
$$

for all $j=1,2, \ldots, d_{p}$. Similarly the basis functions $\psi_{j}^{p L}$ of $\pi^{p}$ with respect to the restricted left regular coaction may be defined as a set of $d_{p}$ elements of $\mathcal{B}$ that have the property that

$$
\pi_{\mathcal{B}}^{L}\left(\psi_{j}^{p L}\right)=\sum_{k=1}^{d_{p}} \psi_{k}^{p L} \otimes \pi_{k j}^{p}
$$

for all $j=1,2, \ldots, d_{p}$. In both cases the matrix coefficients $\pi_{k j}^{p}$ are elements of $\mathcal{A}$, and need not be members of $\mathcal{B}$. 
By contrast with the unrestricted coaction situation, in neither case is there any guarantee that for a given corepresentation $\pi^{p}$ there actually exist basis functions for restricted coactions. For example, in the restricted right regular coaction case, a set of basis functions is provided (for any fixed choice of $\ell=1,2, \ldots$ ) by

$$
\psi_{j}^{p R}=\pi_{\ell j}^{p}
$$

for all $j=1,2, \ldots, d_{p}$ only if $\pi_{\ell j}^{p}$ is an element of $\mathcal{B}$ for some $j=1,2, \ldots, d_{p}$. (Then (I.43) and the fact that $\mathcal{B}$ is assumed in this context to be a right coideal of $\mathcal{A}$ imply that $\pi_{\ell j}^{p} \in \mathcal{B}$ for all $\left.j=1,2, \ldots, d_{p}\right)$. Similarly, in the restricted left regular coaction case, an example is provided (for any fixed choice of $\ell=1,2, \ldots$ ) by

$$
\psi_{j}^{p L}=S^{-2}\left(\pi_{j \ell}^{p \star}\right)
$$

for all $j=1,2, \ldots, d_{p}$ only if $\pi_{j \ell}^{p}$ is an element of $\mathcal{B}$ for some $j=1,2, \ldots, d_{p}$. (Then (I.43) and the fact that $\mathcal{B}$ is assumed in this context to be a $S^{2}$-invariant left coideal and $\star$-subalgebra of $\mathcal{A}$ imply that $S^{-2}\left(\pi_{j \ell}^{p \star}\right) \in \mathcal{B}$ for all $\left.j=1,2, \ldots, d_{p}\right)$.

One very useful result, proved in the same way as the corresponding unrestricted identity (98), is that if $\psi_{k}^{q L}$ exists then

$$
\pi_{\mathcal{B}}^{L}\left(\left(S^{2}\left(\psi_{k}^{q L}\right)\right)^{\star}\right)=\sum_{t=1}^{d_{p}}\left(S^{2}\left(\psi_{t}^{q L}\right)\right)^{\star} \otimes \pi_{t k}^{q \star}
$$

for all $k=1,2, \ldots, d_{p}$.

The orthogonality properties of basis functions are the essentially the same as for those for the unrestricted case that have been given in (99), (100), and (103). (The only qualification is that now it has to be assumed that the relevant matrix corepresentation coefficients are members of $\mathcal{B})$.

If the basis functions $\psi_{k}^{q X}$ and $\phi_{j}^{p X}$ are members of $\mathcal{B}$ (so that they are basis functions for the restricted coactions), then their products $M\left(\psi_{k}^{q X} \otimes \phi_{j}^{p X}\right)$ are also members of $\mathcal{B}$. Consequently the analysis of Subsection V.D goes through without modification, except that all basis functions involved (including the $\theta_{\ell}^{r, \alpha X}$ ) are in $\mathcal{B}$ and one can always replace 
the unrestricted coactions $\pi_{\mathcal{A}}^{X}$ by the restricted coactions $\pi_{\mathcal{B}}^{X}$. The rest of discussion of Section V on tensor products and Clebsch-Gordan coefficients still applies in its entirety.

\section{The irreducible tensor operators in the restricted corepresentation formalisms}

\section{Introduction}

Let $\pi^{q}$ be a unitary irreducible right coaction of $\mathcal{A}$ of dimension $d_{q}$ with matrix coefficients $\pi_{j k}^{q}$. It will be shown that within both the restricted right and the restricted left regular corepresentation formalisms there exist two types of irreducible tensor operators that both belong to this corepresentation $\pi^{q}$. These will be denoted by $Q_{j \mathcal{B}}^{q X}$ and $\widetilde{Q}_{j \mathcal{B}}^{q X}$ (for $j=1,2, \ldots, d_{q}$ and for $X=R$ or $\left.L\right)$, and will be called ordinary and twisted irreducible tensor operators associated with $\mathcal{B}$ respectively. These operators are members of $\mathcal{L}(\mathcal{B})$, which is the set of linear operators that map $\mathcal{B}$ into $\mathcal{B}$. Naturally the two types of irreducible tensor operators coincide in the special case in which $\mathcal{A}$ is commutative. As much of the analysis is the same as for the unrestricted case, all the proofs will be omitted.

2. Definition of the ordinary irreducible tensor operators $Q_{j \mathcal{B}}^{q X}$ and twisted irreducible tensor

$$
\text { operators } \widetilde{Q}_{j \mathcal{B}}^{q X}
$$

The ordinary irreducible tensor operators $Q_{j \mathcal{B}}^{q X}$ belonging to the unitary irreducible right coaction $\pi^{q}$ of $\mathcal{A}$ are defined (for $X=L, R$ ) to be members of $\mathcal{L}(\mathcal{B})$ that satisfy the condition

$$
\left((i d \otimes M) \circ\left(\pi_{\mathcal{B}}^{X} \otimes i d\right) \circ\left(Q_{j \mathcal{B}}^{q X} \otimes S\right) \circ \pi_{\mathcal{B}}^{X}\right)(b)=\sum_{k=1}^{d_{q}} Q_{k \mathcal{B}}^{q X}(b) \otimes \pi_{k j}^{q}
$$

for all $b \in \mathcal{B}$ and all $j=1,2, \ldots, d_{q}$.

The twisted irreducible tensor operators $\widetilde{Q}_{j \mathcal{B}}^{q X}$ belonging to the unitary irreducible right coaction $\pi^{q}$ of $\mathcal{A}$ are defined (for $X=L, R$ ) to be members of $\mathcal{L}(\mathcal{B})$ that satisfy the condition

$$
\left((i d \otimes M) \circ(i d \otimes \sigma) \circ\left(\pi_{\mathcal{B}}^{X} \otimes i d\right) \circ\left(\widetilde{Q}_{j \mathcal{B}}^{q X} \otimes S^{-1}\right) \circ \pi_{\mathcal{B}}^{X}\right)(b)=\sum_{k=1}^{d_{q}} \widetilde{Q}_{k \mathcal{B}}^{q X}(b) \otimes \pi_{k j}^{q}
$$


for all $b \in \mathcal{B}$ and all $j=1,2, \ldots, d_{q}$. (This definition 200) differs from the corresponding definition (199) only in the replacement of $M$ by $M \circ \sigma$ and $S$ by $S^{-1}$ (neither of which have any effect in the special case in which $\mathcal{A}$ is commutative)).

3. Properties of the irreducible tensor operators associated with quantum homogeneous spaces

1. Suppose that $Q$ is the identity operator $i d$ of $\mathcal{L}(\mathcal{B})$ (so that $Q(b)=b$ for all $b \in \mathcal{B}$ ). Then $i d$ is both an ordinary and a twisted irreducible tensor operator for the one-dimensional identity corepresentation of $\mathcal{A}$ (whose sole matrix coefficient is $1_{\mathcal{A}}$ ) in the restricted right regular corepresentation formalism, as well as being a both an ordinary and a twisted irreducible tensor operator for this identity corepresentation in the restricted left regular corepresentation formalism.

2. Suppose now that $\psi_{j}^{q R}$ and $\psi_{j}^{q L}$ are sets of basis functions for $\pi^{q}$, as defined in (194) and (195) respectively (so that they are members of $\mathcal{B}$ ), and suppose that the operators $Q_{j \mathcal{B}}^{q X}$ and $\widetilde{Q}_{j \mathcal{B}}^{q X}$ are defined by

$$
\left.\begin{array}{rl}
Q_{j \mathcal{B}}^{q R}(b) & =M\left(\psi_{j}^{q R} \otimes b\right), \\
\widetilde{Q}_{j \mathcal{B}}^{q R}(b) & =M\left(b \otimes \psi_{j}^{q R}\right), \\
Q_{j \mathcal{B}}^{q L}(b) & =M\left(b \otimes \psi_{j}^{q L}\right), \\
\widetilde{Q}_{j \mathcal{B}}^{q L}(b) & =M\left(\psi_{j}^{q L} \otimes b\right),
\end{array}\right\}
$$

for all $b \in \mathcal{B}$. Then $Q_{j \mathcal{B}}^{q R}, \widetilde{Q}_{j \mathcal{B}}^{q R}, Q_{j \mathcal{B}}^{q L}$, and $\widetilde{Q}_{j \mathcal{B}}^{q L}$ are irreducible tensor operators belonging to $\pi^{q}$.

\section{E. Wigner-Eckart type theorems associated with quantum homogeneous spaces}

If $\pi^{p}, \pi^{q}$, and $\pi^{r}$ are unitary irreducible corepresentations of $\mathcal{A}$ of dimensions $d_{p}, d_{q}$, and $d_{r}$ respectively, $\phi_{j}^{p X}$ and $\psi_{\ell}^{r X}$ are basis functions belonging $\pi^{p}$ and $\pi^{r}$ (with $\phi_{j}^{p X}$ and $\psi_{\ell}^{r X}$ being assumed here to be members of $\mathcal{B}$ ), and $Q_{k \mathcal{B}}^{q X}$ is an ordinary irreducible tensor operator belonging to $\pi^{q}$ (with respect to the relevant restricted regular coaction), then 


$$
\left(\psi_{\ell}^{r X}, Q_{k \mathcal{B}}^{q X}\left(\phi_{j}^{p X}\right)\right)^{X}=\sum_{\alpha=1}^{n_{q p}^{r}}\left(\begin{array}{ll|ll}
r, & \alpha & q & p \\
\ell & k & j
\end{array}\right)\left(r\left|Q_{\mathcal{B}}^{q X}\right| p\right)_{\alpha},
$$

for $X=R$ and $L$, all $j=1,2, \ldots, d_{p}$, all $k=1,2, \ldots, d_{q}$, and all $\ell=1,2, \ldots, d_{r}$. Here the reduced matrix elements $\left(r\left|Q_{\mathcal{B}}^{q X}\right| p\right)_{\alpha}$ are given by

$$
\begin{aligned}
\left(r\left|Q_{\mathcal{B}}^{q X}\right| p\right)_{\alpha} & = \\
& \sum_{s=1}^{d_{p}} \sum_{t=1}^{d_{q}} \sum_{u, v=1}^{d_{r}}\left(\psi_{u}^{r X}, Q_{t \mathcal{B}}^{q X}\left(\phi_{s}^{p X}\right)\right)^{X}\left(\begin{array}{cc|c}
q & p & r, \alpha \\
t & s & v
\end{array}\right) \\
& \times\left\{\left(\left(\mathbf{F}^{r}\right)^{-1}\right)_{v u} / \operatorname{tr}\left(\left(\mathbf{F}^{r}\right)^{-1}\right)\right\}
\end{aligned}
$$

for $\alpha=1,2, \ldots, n_{q p}^{r}$, the inner products $(,)^{R}$ and $(,)^{L}$ are defined in (192) and (193), and $\mathbf{F}^{r}$ is defined in (66).

On the other hand, if $\widetilde{Q}_{k \mathcal{B}}^{q X}$ is a twisted irreducible tensor operator belonging to $\pi^{q}$ (with respect to the relevant restricted regular coaction), then

$$
\left(\psi_{\ell}^{r X}, \widetilde{Q}_{k \mathcal{B}}^{q X}\left(\phi_{j}^{p X}\right)\right)^{X}=\sum_{\alpha=1}^{n_{p q}^{r}}\left(\begin{array}{ll|ll}
r, & \alpha & p & q \\
\ell & j & k
\end{array}\right)\left(r\left|\widetilde{Q}_{\mathcal{B}}^{q X}\right| p\right)_{\alpha},
$$

for $X=R$ and $L$, all $j=1,2, \ldots, d_{p}$, all $k=1,2, \ldots, d_{q}$, and all $\ell=1,2, \ldots, d_{r}$, where the reduced matrix elements $\left(r\left|\widetilde{Q}_{\mathcal{B}}^{q X}\right| p\right)_{\alpha}$ are given by

$$
\begin{aligned}
\left(r\left|\widetilde{Q}_{\mathcal{B}}^{q X}\right| p\right)_{\alpha} & = \\
& \sum_{s=1}^{d_{p}} \sum_{t=1}^{d_{q}} \sum_{u, v=1}^{d_{r}}\left(\psi_{u}^{r X}, \widetilde{Q}_{t \mathcal{B}}^{q X}\left(\phi_{s}^{p X}\right)\right)^{X}\left(\begin{array}{cc|c}
p & q & r, \alpha \\
s & t & v
\end{array}\right) \\
& \times\left\{\left(\left(\mathbf{F}^{r}\right)^{-1}\right)_{v u} / \operatorname{tr}\left(\left(\mathbf{F}^{r}\right)^{-1}\right)\right\}
\end{aligned}
$$

for $\alpha=1,2, \ldots, n_{p q}^{r}$.

These results (202) and (204) demonstrate that again the $j, k$, and $\ell$ dependences of the inner products $\left(\psi_{\ell}^{r X}, Q_{k \mathcal{B}}^{q X} \phi_{j}^{p X}\right)^{X}$ and $\left(\psi_{\ell}^{r X}, \widetilde{Q}_{k \mathcal{B}}^{q X} \phi_{j}^{p X}\right)^{X}$ are determined only by ClebschGordan coefficients, and so they have the same form as in the classic Wigner-Eckart theorem. (However, it should be noted that in the general case in which $\mathcal{A}$ is non-commutative, the inner products for the ordinary and twisted irreducible tensor operators involve different sets of Clebsch-Gordan coefficients). 
As the proofs of (202) and (204) follow the same lines as in the unrestricted case considered in Section VII, they will be omitted here.

\section{F. Products of irreducible tensor operators associated with quantum homogeneous spaces}

The arguments of Section VIII can be applied (with $\mathcal{A}$ replaced by $\mathcal{B}$ ) to show that

$$
Q_{\ell \mathcal{B}}^{r X, \alpha}=\sum_{j=1}^{d_{p}} \sum_{k=1}^{d_{q}}\left(\begin{array}{cc|cc}
p & q & r & , \alpha \\
j & k & \ell
\end{array}\right) Q_{j \mathcal{B}}^{p X} Q_{k \mathcal{B}}^{q X},
$$

for $\ell=1,2, \ldots, d_{r}$, and $\alpha=1,2, \ldots, n_{p q}^{r}$. Here $Q_{\ell \mathcal{B}}^{r X, \alpha}\left(\right.$ for $\left.\alpha=1,2, \ldots, n_{p q}^{r}\right)$ are $n_{p q}^{r}$ ordinary irreducible tensor operators belonging to $\pi^{r}$ that are, in general, all different. Moreover,

$$
\widetilde{Q}_{\ell \mathcal{B}}^{r X, \alpha}=\sum_{j=1}^{d_{p}} \sum_{k=1}^{d_{q}}\left(\begin{array}{ll|l}
q & p & r, \alpha \\
k & j & \ell
\end{array}\right) \widetilde{Q}_{j \mathcal{B}}^{p X} \widetilde{Q}_{k \mathcal{B}}^{q X},
$$

for $\ell=1,2, \ldots, d_{r}$, and $\alpha=1,2, \ldots, n_{q p}^{r}$, where $\widetilde{Q}_{\ell \mathcal{B}}^{r X, \alpha}\left(\right.$ for $\left.\alpha=1,2, \ldots, n_{q p}^{r}\right)$ are $n_{q p}^{r}$ twisted irreducible tensor operators belonging to $\pi^{r}$ that are, in general, all different.

\section{ACKNOWLEDGMENTS}

This work was started while the author was on research leave at the Sektion Physik of the Ludwig-Maximilians-Universität, München. The author is grateful to Professor J. Wess and his colleagues for their hospitality and to the Deutscher Akademischer Austauschdienst for its financial support.

\section{APPENDIX A: INTRODUCTION}

The purpose of this Appendix is to motivate the definitions that are given in the main body of the paper for the irreducible tensor operators and the projection operators. This will be done by considering the simple special case in which the Hopf algebra $\mathcal{A}$ is the set 
of functions defined on a finite group $\mathcal{G}$ of order $g$, so that the dual $\mathcal{A}^{\prime}$ of $\mathcal{A}$ is the group algebra of $\mathcal{G}$. In this situation both $\mathcal{A}$ and $\mathcal{A}^{\prime}$ are of finite dimension $g$. Of course, as $\mathcal{A}$ is commutative in this special case, the resulting expressions are to some extent ambiguous, in that in this special case $M$ is indistinguishable from $M \circ \sigma$ and $S$ is indistinguishable from $S^{-1}$. The demonstration of the correctness, consistency, and usefulness of the definitions that are actually employed for the general case are the subject matter of the self-contained arguments of the main body of this paper.

To proceed with this motivation, it is necessary to start with some well- known facts concerning the relationship of $\mathcal{A}$ and $\mathcal{A}^{\prime}$. It is easily shown that $\mathcal{A}^{\prime}$ is also a Hopf algebra, whose multiplication operation $M_{\mathcal{A}^{\prime}}$, comultiplication operation $\Delta_{\mathcal{A}^{\prime}}$, and antipode $S_{\mathcal{A}^{\prime}}$ are related to those of $\mathcal{A}$ by

$$
\left\langle M_{\mathcal{A}^{\prime}}\left(a^{\prime} \otimes b^{\prime}\right), a\right\rangle=\left\langle\left(a^{\prime} \otimes b^{\prime}\right), \Delta(a)\right\rangle
$$

for all $a \in \mathcal{A}$ and all $a^{\prime}, b^{\prime} \in \mathcal{A}^{\prime}$,

$$
\left\langle\Delta_{\mathcal{A}^{\prime}}\left(a^{\prime}\right),(a \otimes b)\right\rangle=\left\langle a^{\prime}, M(a \otimes b)\right\rangle
$$

for all $a, b \in \mathcal{A}$ and all $a^{\prime} \in \mathcal{A}^{\prime}$, and

$$
\left\langle S_{\mathcal{A}^{\prime}}\left(a^{\prime}\right), a\right\rangle=\left\langle a^{\prime}, S(a)\right\rangle
$$

for all $a \in \mathcal{A}$ and all $a^{\prime} \in \mathcal{A}^{\prime}$.

Now suppose that $\pi_{V}$ is a right coaction of $\mathcal{A}$ with carrier space $V$. Then there exists a corresponding left action $\pi_{V}^{\prime}$ of $\mathcal{A}^{\prime}$ with the same carrier space $V$. This is a linear mapping from $\mathcal{A}^{\prime} \otimes \mathcal{V}$ to $V$ such that

$$
\pi_{V}^{\prime} \circ\left(i d \otimes \pi_{V}^{\prime}\right)=\pi_{V}^{\prime} \circ\left(M_{\mathcal{A}^{\prime}} \otimes i d\right)
$$

and

$$
\pi_{V}^{\prime} \circ\left(u_{\mathcal{A}^{\prime}} \otimes i d\right)=M_{\mathbb{C}, V}
$$


where $u_{\mathcal{A}^{\prime}}$ is the unit operator of $\mathcal{A}^{\prime}$, and $M_{\mathbb{C}, V}(z \otimes v)=z v$ for all $v \in V$ and all $z \in \mathbb{C}$. The relationship between $\pi_{V}$ and $\pi_{V}^{\prime}$ can be expressed as

$$
\pi_{V}^{\prime}\left(a^{\prime} \otimes v\right)=\sum_{[v]} v_{[1]}\left\langle a^{\prime}, v_{[2]}\right\rangle
$$

for all $a^{\prime} \in \mathcal{A}^{\prime}$ and all $v \in V$, where the notation of (48) has been employed, or equivalently as

$$
\pi_{V}^{\prime}\left(a^{\prime} \otimes v\right)=\left(M_{V, \boldsymbol{C}} \circ(i d \otimes e v) \circ(\sigma \otimes i d) \circ\left(i d \otimes \pi_{V}\right)\right)\left(a^{\prime} \otimes v\right)
$$

for all $a^{\prime} \in \mathcal{A}^{\prime}$ and all $v \in V$, where the evaluation map $e v$ of (41) has been used. The inverse of these is

$$
\pi_{V}(v)=\sum_{j} \pi_{V}^{\prime}\left(a^{j} \otimes v\right) \otimes a_{j},
$$

for all $v \in V$, where the basis of $\mathcal{A}$ and the dual basis of $\mathcal{A}^{\prime}$ appear, and are assumed to be such that (42) holds. If $V$ is of dimension $d$ with basis elements $v_{1}, v_{2}, \ldots, v_{d}$, the matrix elements $\pi^{\prime}\left(a^{\prime}\right)_{j k}$ of the representation are such that

$$
\pi_{V}^{\prime}\left(a^{\prime} \otimes v_{j}\right)=\sum_{k=1}^{d} \pi^{\prime}\left(a^{\prime}\right)_{k j} v_{k}
$$

for all $a^{\prime} \in \mathcal{A}^{\prime}$ and $j=1,2, \ldots, d$. It then follows from (A8) that these representation matrix elements $\pi^{\prime}\left(a^{\prime}\right)_{j k}$ are related to the corepresentation matrix coefficients of $\pi_{j k}^{V}$ of (45) by

$$
\pi^{\prime}\left(a^{\prime}\right)_{j k}=\left\langle a^{\prime}, \pi_{j k}^{V}\right\rangle
$$

for all $a^{\prime} \in \mathcal{A}^{\prime}$ and $j, k=1,2, \ldots, d$.

The right regular action $R$ of $\mathcal{G}$ is defined by

$$
(R(x \otimes f))(y)=f(y x),
$$

for all elements $x, y \in \mathcal{G}$ and all functions $f$ defined on $\mathcal{G}$, where $y x$ is evaluated using the group multiplication operation of $\mathcal{G}$. This definition (A11) can be immediately extended to all $x, y \in \mathcal{A}^{\prime}$, with $f$ being any element of $\mathcal{A}$. It is then easy to verify that $R$ is a left action 
of $\mathcal{A}^{\prime}$ with carrier space $\mathcal{A}$, and, using (A6) (or its equivalents), that it is the left action that corresponds to the right regular coaction $\pi_{\mathcal{A}}^{R}$ of $\mathcal{A}$ that was defined in (80). Then, by (A7),

$$
R(x \otimes f)=\left(M_{\mathcal{A}, C^{*}} \circ(i d \otimes e v) \circ(\sigma \otimes i d) \circ(i d \otimes \Delta)\right)(x \otimes f)
$$

for all $x \in \mathcal{A}^{\prime}$ and all $f \in \mathcal{A}$, and also, by (80) and (A6),

$$
\langle y, R(x \otimes f)\rangle=\langle y \otimes x, \Delta(f)\rangle=\left\langle y \otimes x, \pi_{\mathcal{A}}^{R}(f)\right\rangle
$$

for all $x, y \in \mathcal{A}^{\prime}$ and all $f \in \mathcal{A}$. The content of (A11) can usefully be re-expressed as

$$
\widehat{R}(x) f(y)=f(y x)
$$

by introducing an operator $\widehat{R}(x)$ for each $x \in \mathcal{A}^{\prime}$.

Similarly, the left regular action $L$ of $\mathcal{G}$ is defined by

$$
(L(x \otimes f))(y)=f\left(x^{-1} y\right)
$$

for all elements $x, y \in \mathcal{G}$ and all functions $f$ defined on $\mathcal{G}$, which immediately extends to all $x, y \in \mathcal{A}^{\prime}$ and all $f \in \mathcal{A}$. Then $L$ is the left action of $\mathcal{A}^{\prime}$ that corresponds to the left regular coaction $\pi_{\mathcal{A}}^{L}$ of $\mathcal{A}$ that was defined in (81). Thus, by (A7),

$$
L(x \otimes f)=\left(M_{\mathcal{A}, \boldsymbol{C}} \circ(i d \otimes e v) \circ(\sigma \otimes i d) \circ\left(i d \otimes \pi_{\mathcal{A}}^{L}\right)\right)(x \otimes f)
$$

for all $x \in \mathcal{A}^{\prime}$ and all $f \in \mathcal{A}$, and also, by (81) and (6),

$$
\langle y, L(x \otimes f)\rangle=\langle y \otimes x,(\sigma \circ(S \otimes i d) \circ \Delta)(f)\rangle=\left\langle y \otimes x, \pi_{\mathcal{A}}^{L}(f)\right\rangle
$$

for all $x, y \in \mathcal{A}^{\prime}$ and all $f \in \mathcal{A}$. Moreover, (A15) can be re-written as

$$
\widehat{L}(x) f(y)=f\left(S_{\mathcal{A}^{\prime}}(x) y\right)
$$

by introducing an operator $\widehat{L}(x)$ for each $x \in \mathcal{A}^{\prime}$. 


\section{APPENDIX B: IRREDUCIBLE TENSOR OPERATORS}

\section{Outline of argument}

The first stage is to recall the definition of irreducible tensor operators in the group theoretical context. The next stage is to cast these considerations into the language of Hopf algebras, and the final stage is to put them into a form in which they involve quantities belonging only to $\mathcal{A}$. The argument will be given first for the right regular situation, and then for the left regular situation. In each case the definitions of irreducible tensor operators will be deduced first, and motivation for the right coactions that appear in the consistency arguments will follow. (The diagrammatic method that is described, for example, by Majid ${ }^{37}$, was employed to deduce the proofs that follow, but for typographical convenience these proofs have been transcribed here into the usual purely symbolic form).

\section{Irreducible tensor operators in the group theoretical right regular representation formalism}

a. Derivations of the conditions for the $Q_{j}^{q R}$ and $\widetilde{Q}_{j}^{q R}$

Let $\Gamma^{q}$ be a $d_{q}$ dimensional representation of $\mathcal{G}$. Then the irreducible tensor operators $Q_{j}^{q R}$ may be defined to act on functions defined on $\mathcal{G}$ in such a way that

$$
\widehat{R}(x) Q_{j}^{q R} \widehat{R}\left(x^{-1}\right)=\sum_{k=1}^{d_{q}} \Gamma_{k j}^{q}(x) Q_{k}^{q R}
$$

for all $x \in \mathcal{G}$ and $j=1,2, \ldots, d_{q}$, or, more explicitly, such that

$$
\left(\widehat{R}(x) Q_{j}^{q R} \widehat{R}\left(x^{-1}\right)\right)(f(y))=\sum_{k=1}^{d_{q}} \Gamma_{k j}^{q}(x)\left(Q_{k}^{q R}(f)\right)(y),
$$

for all $x, y \in \mathcal{G}$, for all functions $f$ defined on $\mathcal{G}$, and $j=1,2, \ldots, d_{q}$. Now define $\widehat{\pi}_{\mathcal{L}(\mathcal{A})}^{R \prime}(x)$ by

$$
\widehat{\pi}_{\mathcal{L}(\mathcal{A})}^{R \prime}(x)(Q)=\widehat{R}(x) Q \widehat{R}\left(x^{-1}\right)
$$


for all $x \in \mathcal{G}$ and for all linear operators $Q$ that act on functions defined on $\mathcal{G}$, so that (B1) becomes

$$
\widehat{\pi}_{\mathcal{L}(\mathcal{A})}^{R \prime}(x)\left(Q_{j}^{q R}\right)=\sum_{k=1}^{d_{q}} \Gamma_{k j}^{q}(x) Q_{k}^{q R},
$$

for all $x \in \mathcal{G}$ and $j=1,2, \ldots, d_{q}$. As discussed previously in Section I (in only a slightly different context), the consistency of the definition (B4) is consequence of the assumption that $\Gamma^{q}$ is a representation of $\mathcal{G}$ and the fact that

$$
\widehat{\pi}_{\mathcal{L}(\mathcal{A})}^{R \prime}(x y)=\widehat{\pi}_{\mathcal{L}(\mathcal{A})}^{R \prime}(x) \widehat{\pi}_{\mathcal{L}(\mathcal{A})}^{R \prime}(y)
$$

for all $x, y \in \mathcal{G}$.

As

$$
\Delta_{\mathcal{A}^{\prime}}(x)=x \otimes x, S_{\mathcal{A}^{\prime}}(x)=x^{-1}
$$

for all $x \in \mathcal{G}$, it follows from (A14) and (A11) that

$$
\begin{aligned}
& \left(\widehat{\pi}_{\mathcal{L}(\mathcal{A})}^{{ }^{\prime}}(x)\left(Q_{j}^{q R}\right)\right) f(y)= \\
& \left(e v \circ(i d \otimes R) \circ\left(i d \otimes i d \otimes Q_{j}^{q R}\right) \circ(i d \otimes i d \otimes R) \circ\left(i d \otimes i d \otimes S_{\mathcal{A}^{\prime}} \otimes i d\right)\right. \\
& \left.\circ\left(i d \otimes \Delta_{\mathcal{A}^{\prime}} \otimes i d\right)\right)(y \otimes x \otimes f)
\end{aligned}
$$

which is now well- defined for all $x, y \in \mathcal{A}^{\prime}$, for all $f \in \mathcal{A}$, and $Q_{j}^{q R} \in \mathcal{L}(\mathcal{A})$. Thus, by (A12),

$$
\begin{aligned}
& \left(\widehat{\pi}_{\mathcal{L}^{\prime}(\mathcal{A})}^{{ }^{\prime}}(x)\left(Q_{j}^{q R}\right)\right) f(y)= \\
& \left(e v \circ\left(i d \otimes M_{\mathcal{A}, \boldsymbol{C}^{\prime}}\right) \circ(i d \otimes i d \otimes e v) \circ(i d \otimes \sigma \otimes i d) \circ(i d \otimes i d \otimes \Delta)\right. \\
& \circ\left(i d \otimes i d \otimes Q_{j}^{q R}\right) \circ\left(i d \otimes i d \otimes M_{\mathcal{A}, \boldsymbol{C}}\right) \circ(i d \otimes i d \otimes i d \otimes e v) \\
& \circ(i d \otimes i d \otimes \sigma \otimes i d) \circ(i d \otimes i d \otimes i d \otimes \Delta) \circ\left(i d \otimes i d \otimes S_{\mathcal{A}^{\prime}} \otimes i d\right) \\
& \left.\circ\left(i d \otimes \Delta_{\mathcal{A}^{\prime}} \otimes i d\right)\right)(y \otimes x \otimes f),
\end{aligned}
$$

which reduces, by (A3), to

$$
\begin{aligned}
& \left(\widehat{\pi}_{\mathcal{L}(\mathcal{A})}^{R \prime}(x)\left(Q_{j}^{q R}\right)\right) f(y)= \\
& \left(M_{\boldsymbol{C}^{\prime}} \circ\left(e v \otimes M_{\boldsymbol{C}^{\prime}}\right) \circ(i d \otimes \sigma \otimes i d) \circ(i d \otimes e v \otimes i d \otimes i d)\right. \\
& \circ\left\{i d \otimes i d \otimes\left(\sigma \circ \Delta \circ Q_{j}^{q R}\right) \otimes i d\right\} \circ(i d \otimes i d \otimes \sigma) \circ(i d \otimes i d \otimes e v \otimes i d) \\
& \left.\circ(i d \otimes i d \otimes i d \otimes S \otimes i d) \circ\left(i d \otimes \Delta_{\mathcal{A}^{\prime}} \otimes \sigma\right) \circ(i d \otimes i d \otimes \Delta)\right)(y \otimes x \otimes f),
\end{aligned}
$$


However, (A2) implies that

$$
(e v \circ(i d \otimes M) \circ(i d \otimes \sigma))(x \otimes a \otimes b)=\left(M_{\mathbb{C}^{\circ}} \circ(e v \otimes e v) \circ\left(\Delta_{\mathcal{A}^{\prime}} \otimes i d \otimes i d\right)(x \otimes a \otimes b)\right.
$$

for all $a, b \in \mathcal{A}$ and all $x \in \mathcal{A}^{\prime}$, so $(\mathrm{B} 9)$ reduces to

$$
\begin{aligned}
& \left(\widehat{\pi}_{\mathcal{L}(\mathcal{A})}^{R \prime}(x)\left(Q_{j}^{q R}\right)\right) f(y) \\
& =\left(M_{\mathbb{C}^{\prime}} \circ(e v \otimes e v) \circ(i d \otimes \sigma \otimes M) \circ(i d \otimes i d \otimes \Delta \otimes i d)\right. \\
& \left.\circ\left(i d \otimes i d \otimes Q_{j}^{q R} \otimes S\right) \circ(i d \otimes i d \otimes \Delta)\right)(y \otimes x \otimes f)
\end{aligned}
$$

and hence

$$
\begin{aligned}
& \left(\widehat{\pi}_{\mathcal{L}^{\prime}(\mathcal{A})}^{\prime \prime}(x)\left(Q_{j}^{q R}\right)\right) f(y)= \\
& \left(M_{\mathbb{C}^{\circ}} \circ(e v \otimes e v) \circ(i d \otimes \sigma \otimes i d) \circ(i d \otimes i d \otimes i d \otimes M) \circ(i d \otimes i d \otimes \Delta \otimes i d)\right. \\
& \left.\circ\left(i d \otimes i d \otimes Q_{j}^{q R} \otimes S\right) \circ(i d \otimes i d \otimes \Delta) \circ\left(i d \otimes i d \otimes M_{\mathcal{A}, \mathbb{C}}\right)\right)(y \otimes x \otimes f \otimes z)
\end{aligned}
$$

for all $x, y \in \mathcal{A}^{\prime}$, all $f \in \mathcal{A}, Q_{j}^{q R} \in \mathcal{L}(\mathcal{A})$, and all $z \in \mathbb{C}$.

Turning to the right-hand side of (B4), by (A10),

$$
\sum_{k=1}^{d_{q}} \Gamma_{k j}^{q}(x)\left(Q_{k}^{q R}(f)\right)(y)=\sum_{k=1}^{d_{q}}\left\langle x, \pi_{k j}^{q}\right\rangle\left\langle y, Q_{k}^{q R}\right\rangle,
$$

for all $x, y \in \mathcal{A}^{\prime}$, and $j=1,2, \ldots, d_{q}$, and hence

$$
\begin{aligned}
& \sum_{k=1}^{d_{q}} \Gamma_{k j}^{q}(x)\left(Q_{k}^{q R}(f)\right)(y)= \\
& \sum_{k=1}^{d_{q}}\left(M_{C^{c}} \circ(e v \otimes e v) \circ(i d \otimes \sigma \otimes i d) \circ\left(i d \otimes i d \otimes Q_{k}^{q R} \otimes \pi_{k j}^{q}\right)\right. \\
& \circ(i d \otimes i d \otimes i d \otimes u))(y \otimes x \otimes f \otimes z)
\end{aligned}
$$

for all $x, y \in \mathcal{A}^{\prime}$, all $f \in \mathcal{A}, Q_{k}^{q R} \in \mathcal{L}(\mathcal{A})$, and all $z \in \mathbb{C}$.

Now (B4) implies that the expressions on the right-hand sides of (B12) and (B13) may be equated. As the first three factors of each, namely $M_{\mathbb{C}^{*}} \circ(e v \otimes e v) \circ(i d \otimes \sigma \otimes i d)$, are the same, the equality holds with these removed. However, on both sides of this new equality, the factor $y \otimes x$ is only acted on by a succession of identity operators of the form $i d \otimes i d$. Consequently both $y \otimes x$ and these identity operators can be removed, leaving the result that $(\mathbb{B} 1)$ is equivalent to the condition 


$$
\begin{aligned}
& \left((i d \otimes M) \circ(\Delta \otimes i d) \circ\left(Q_{j}^{q R} \otimes S\right) \circ \Delta \circ M_{\mathcal{A}, \mathbb{C}^{\prime}}\right)(f \otimes z)= \\
& \quad \sum_{k=1}^{d_{q}}\left(\left(Q_{k}^{q R} \otimes \pi_{k j}^{q}\right) \circ(i d \otimes u)\right)(f \otimes z)
\end{aligned}
$$

for all $f \in \mathcal{A}$, all $j=1,2, \ldots, d_{q}$, and all $z \in \mathbb{C}$. This can be rewritten as

$$
\left((i d \otimes M) \circ(\Delta \otimes i d) \circ\left(Q_{j}^{q R} \otimes S\right) \circ \Delta\right)(f)=\sum_{k=1}^{d_{q}} Q_{k}^{q R}(f) \otimes \pi_{k j}^{q}
$$

for all $f \in \mathcal{A}$ and all $j=1,2, \ldots, d_{q}$, which is the condition (148).

Because $M$ is indistinguishable from $M \circ \sigma$ and $S$ is indistinguishable from $S^{-1}$ in the situation being considered here, the above arguments would equally well apply with each of the following 3 substitutions:

1. replace $M$ by $M \circ \sigma$, but leave $S$ unchanged;

2. leave $M$ unchanged, but replace $S$ by $S^{-1}$;

3. replace $M$ by $M \circ \sigma$ and replace $S$ by $S^{-1}$.

However, in the general case in which $\mathcal{A}$ is non- commutative, the possibilities (1) and (2) are excluded because with them the identity operator would not be an irreducible tensor operator belonging to the identity corepresentation. Of course, with the substitution (3), (148) changes into (155), which is the defining condition for a twisted irreducible tensor operator $\widetilde{Q}_{j}^{q R}$.

b. Derivations of the right coactions $\pi_{\mathcal{L}(\mathcal{A})}^{R}$ and $\widetilde{\pi}_{\mathcal{L}(\mathcal{A})}^{R}$

First recast (B3) as

$$
\pi_{\mathcal{L}(\mathcal{A})}^{R \prime}(x \otimes Q)=\widehat{R}(x) Q \widehat{R}\left(x^{-1}\right)
$$

for all $x \in \mathcal{G}$ and for all linear operators $Q$ that act on functions defined on $\mathcal{G}$, where $\pi_{\mathcal{L}(\mathcal{A})}^{R \prime}$ is a mapping from $\mathcal{A}^{\prime} \otimes \mathcal{L}(\mathcal{A})$ into $\mathcal{L}(\mathcal{A})$. In Hopf algebra language, this can be re-expressed as 


$$
\begin{aligned}
\pi_{\mathcal{L}(\mathcal{A})}^{R \prime}(x \otimes Q)= & (\widehat{M} \circ(i d \otimes \widehat{M}) \circ(\widehat{R} \otimes i d \otimes \widehat{R}) \circ \\
& \left.\circ(i d \otimes \sigma) \circ\left(i d \otimes S_{\mathcal{A}^{\prime}} \otimes i d\right) \circ\left(\Delta_{\mathcal{A}^{\prime}} \otimes i d\right)\right)(x \otimes Q),
\end{aligned}
$$

where $\widehat{M}$ is the operator multiplication operator defined in (179). It is then easily shown that $\pi_{\mathcal{L}(\mathcal{A})}^{R \prime}$ is a left action of $\mathcal{A}^{\prime}$ with carrier space $\mathcal{L}(\mathcal{A})$.

This expression for $\pi_{\mathcal{L}(\mathcal{A})}^{R \prime}$ can be re- expressed in terms of the structure constants introduced in Section II with respect to the basis $a_{1}, a_{2}, \ldots$ of $\mathcal{A}$, and the basis $a^{1}, a^{2}, \ldots$ of its dual $\mathcal{A}^{\prime}$. First define the operators $\mathcal{P}_{j}^{k}$ by

$$
\mathcal{P}_{j}^{k}(a)=\left\langle a^{k}, a\right\rangle a_{j}
$$

(for all $a \in \mathcal{A}$ and all $j, k=1,2, \ldots$ ), and then define the matrix elements $q_{j}^{k}$ of $Q$ by

$$
q_{j}^{k}=\left\langle a^{k}, Q\left(a_{j}\right)\right\rangle
$$

(for all $j, k=1,2, \ldots$ ). Clearly the operators $\mathcal{P}_{j}^{k}$ are members of $\mathcal{L}(\mathcal{A})$, and any operator $Q$ of $\mathcal{L}(\mathcal{A})$ can be expressed as $Q=\sum_{j, k} Q_{k}^{j} \mathcal{P}_{j}^{k}$. Then, by (A13) and (B18),

$$
\widehat{R}\left(a^{m}\right)=\sum_{j, k=1}^{g} \mu_{k}^{j m} \mathcal{P}_{j}^{k}
$$

for all $m=1,2 \ldots, g$. On substituting (B20) into (B17), and using (A2) and (A3), it follows that

$$
\pi_{\mathcal{L}(\mathcal{A})}^{R \prime}\left(a^{m} \otimes Q\right)=\sum_{i, j, k, \ell, u, v, w=1}^{g}\left(m_{u v}^{m} s_{w}^{v} \mu_{i}^{j u} \mu_{k}^{\ell w} q_{\ell}^{i}\right) \mathcal{P}_{j}^{k},
$$

for all $Q \in \mathcal{L}(\mathcal{A})$ and all $m=1,2 \ldots, g$.

Using (A8), the corresponding right coaction $\pi_{\mathcal{L}(\mathcal{A})}^{R}$ of $\mathcal{A}$ with the same carrier space $\mathcal{L}(\mathcal{A})$ is given by

$$
\pi_{\mathcal{L}(\mathcal{A})}^{R}(Q)=\sum_{m=1}^{g} \pi_{\mathcal{L}(\mathcal{A})}^{R \prime}\left(a^{m} \otimes Q\right) \otimes a_{m}
$$

for all $Q \in \mathcal{L}(\mathcal{A})$. Thus

$$
\pi_{\mathcal{L}(\mathcal{A})}^{R}(Q)=\sum_{i, j, k, \ell, m, u, v, w=1}^{g}\left(m_{u v}^{m} s_{w}^{v} \mu_{i}^{j u} \mu_{k}^{\ell w} q_{\ell}^{i}\right)\left(\mathcal{P}_{j}^{k} \otimes a_{m}\right)
$$


for all $Q \in \mathcal{L}(\mathcal{A})$. The final stage is to re-express this in a basis free form. This can be done by writing

$$
\pi_{\mathcal{L}(\mathcal{A})}^{R}(Q)=\sum_{[Q]} Q_{[1]} \otimes Q_{[2]},
$$

where $Q_{[1]} \in \mathcal{L}(\mathcal{A})$ and $Q_{[1]} \in \mathcal{A}$ are such that

$$
\sum_{[Q]} Q_{[1]}(a) \otimes Q_{[2]}=((i d \otimes M) \circ(\Delta \otimes i d) \circ(Q \otimes S) \circ \Delta)(a)
$$

for all $Q \in \mathcal{L}(\mathcal{A})$ and all $a \in \mathcal{A}$.

The right coaction $\tilde{\pi}_{\mathcal{L}(\mathcal{A})}^{R}$ is obtained from $\pi_{\mathcal{L}(\mathcal{A})}^{R}$ by replacing $M$ by $M \circ \sigma$ and replacing $S$ by $S^{-1}$. The right coactions $\pi_{\mathcal{T}(\mathcal{A})}^{R}$ and $\widetilde{\pi}_{\mathcal{T}(\mathcal{A})}^{R}$ of Sections VI.B.1 and VI.B.2 have essentially the same definitions as $\pi_{\mathcal{L}(\mathcal{A})}^{R}$ and $\widetilde{\pi}_{\mathcal{L}(\mathcal{A})}^{R}$, except that their domains are restricted to the appropriate subspaces $\mathcal{T}(\mathcal{A})$.

\section{Irreducible tensor operators in the group theoretical left regular representation formalism}

a. Derivations of the conditions for the $Q_{j}^{q L}$ and $\widetilde{Q}_{j}^{q L}$

The argument for the left regular formalism follows exactly the same line as that for the right regular case given above up to (B7), the only differences being that the operators $\widehat{R}(x)$ must be replaced by the operators $\widehat{L}(x)$, the left action $R$ must be replaced by the left action $L$, and the label $R$ must be replaced by $L$ on the irreducible tensor operators $Q_{j}^{q R}$, on the left action $\pi_{\mathcal{L}(\mathcal{A})}^{R \prime}$, and on the corresponding right coaction $\pi_{\mathcal{L}(\mathcal{A})}^{R}$. Thus, by (A16), the analogue of $(\mathrm{B8})$ is 
$\left(\widehat{\pi}_{\mathcal{L}(\mathcal{A})}^{L \prime}(x)\left(Q_{j}^{q L}\right)\right) f(y)\left(=\widehat{L}(x) Q_{j}^{q L} \widehat{L}\left(x^{-1}\right)\right)=$

$\left(e v \circ\left(i d \otimes M_{\mathcal{A}, \boldsymbol{C}}\right) \circ(i d \otimes i d \otimes e v) \circ(i d \otimes \sigma \otimes i d) \circ(i d \otimes i d \otimes \sigma)\right.$

$\circ(i d \otimes i d \otimes S \otimes i d) \circ(i d \otimes i d \otimes \Delta) \circ\left(i d \otimes i d \otimes Q_{j}^{q L}\right) \circ\left(i d \otimes i d \otimes M_{\mathcal{A}, \boldsymbol{C}}\right)$

$\circ(i d \otimes i d \otimes i d \otimes e v) \circ(i d \otimes i d \otimes \sigma \otimes i d) \circ(i d \otimes i d \otimes i d \otimes \sigma)$

$\circ(i d \otimes i d \otimes i d \otimes S \otimes i d) \circ(i d \otimes i d \otimes i d \otimes \Delta)$

$\left.\circ\left(i d \otimes i d \otimes S_{\mathcal{A}^{\prime}} \otimes i d\right) \circ\left(i d \otimes \Delta_{\mathcal{A}^{\prime}} \otimes i d\right)\right)(y \otimes x \otimes f)$,

which reduces, by (A3), (B10), and (16) to

$$
\begin{aligned}
& \left(\widehat{\pi}_{\mathcal{L}(\mathcal{A})}^{L \prime}(x)\left(Q_{j}^{q L}\right)\right) f(y)= \\
& \left(M_{\mathbb{C}^{C}} \circ(e v \otimes e v) \circ(i d \otimes \sigma \otimes i d) \circ(i d \otimes i d \otimes \sigma) \circ(i d \otimes i d \otimes S \otimes i d)\right. \\
& \circ(i d \otimes i d \otimes M \otimes i d) \circ(i d \otimes i d \otimes i d \otimes \Delta) \circ\left(i d \otimes i d \otimes S \otimes Q_{j}^{q L}\right) \\
& \left.\circ(i d \otimes i d \otimes \Delta) \circ\left(i d \otimes i d \otimes M_{\mathcal{A}, \mathcal{C}}\right)\right)(y \otimes x \otimes f \otimes z)
\end{aligned}
$$

for all $x, y \in \mathcal{A}^{\prime}$, all $f \in \mathcal{A}, Q_{j}^{q L} \in \mathcal{L}(\mathcal{A})$, and all $z \in \mathbb{C}^{\prime}$.

The right-hand side of the irreducible tensor operator definition that corresponds to this is

$$
\begin{aligned}
& \sum_{k=1}^{d_{q}} \Gamma_{k j}^{q}(x)\left(Q_{k}^{q L}(f)\right)(y)= \\
& \sum_{k=1}^{d_{q}}\left(M_{C^{\circ}} \circ(e v \otimes e v) \circ(i d \otimes \sigma \otimes i d) \circ\left(i d \otimes i d \otimes Q_{k}^{q L} \otimes \pi_{k j}^{q}\right)\right. \\
& \circ(i d \otimes i d \otimes i d \otimes u))(y \otimes x \otimes f \otimes z)
\end{aligned}
$$

for all $x, y \in \mathcal{A}^{\prime}$, all $f \in \mathcal{A}, Q_{k}^{q L} \in \mathcal{L}(\mathcal{A})$, and all $z \in \mathbb{C}^{\prime}$.

Equating the right-hand sides (B24) and( $\left(M_{C^{\circ}} \circ(e v \otimes e v) \circ(i d \otimes \sigma \otimes i d)\right)$ of each, and removing the factor $y \otimes x$ and the succession of identity operators of the form $i d \otimes i d$ that act on $y \otimes x$, it follows that the defining condition becomes

$$
\begin{aligned}
& \left(\sigma \circ(S \otimes i d) \circ(M \otimes i d) \circ(i d \otimes \Delta) \circ\left(S \otimes Q_{j}^{q L}\right) \circ \Delta \circ M_{\mathcal{A}, \boldsymbol{C}}\right)(f \otimes z) \\
& =\sum_{k=1}^{d_{q}}\left(\left(Q_{k}^{q L} \otimes \pi_{k j}^{q}\right) \circ(i d \otimes u)\right)(f \otimes z)
\end{aligned}
$$

for all $f \in \mathcal{A}$, all $j=1,2, \ldots, d_{q}$, and all $z \in \mathbb{C}$. This can be rewritten as

$$
\left(\sigma \circ(S \otimes i d) \circ(M \otimes i d) \circ(i d \otimes \Delta) \circ\left(S \otimes Q_{j}^{q L}\right) \circ \Delta\right)(f)=\sum_{k=1}^{d_{q}} Q_{k}^{q L}(f) \otimes \pi_{k j}^{q}
$$


for all $f \in \mathcal{A}$ and all $j=1,2, \ldots, d_{q}$, which is the condition (161).

Because $\Delta_{\mathcal{A}^{\prime}}$ is indistinguishable from $\sigma \circ \Delta_{\mathcal{A}^{\prime}}$ and $S_{\mathcal{A}^{\prime}}$ is indistinguishable from $S_{\mathcal{A}^{\prime}}^{-1}$ in the situation being considered here, the above arguments would equally well apply to (B23) with each of the following 3 substitutions:

1. replace $\Delta_{\mathcal{A}^{\prime}}$ by $\sigma \circ \Delta_{\mathcal{A}^{\prime}}$, but leave $S_{\mathcal{A}^{\prime}}$ unchanged;

2. leave $\Delta_{\mathcal{A}^{\prime}}$ unchanged, but replace $S_{\mathcal{A}^{\prime}}$ by $S_{\mathcal{A}^{\prime}}^{-1}$;

3. (c) replace $\Delta_{\mathcal{A}^{\prime}}$ by $\sigma \circ \Delta_{\mathcal{A}^{\prime}}$ and replace $S_{\mathcal{A}^{\prime}}$ by $S_{\mathcal{A}^{\prime}}^{-1}$.

In each case the $S$ factor in (B23) should be left unchanged because it comes from the definition (81) of the left regular (right) coaction. (Replacing $S$ by $S^{-1}$ in (81) would give another right coaction, but the original one is merely the double contragredient of this). In the general case in which $\mathcal{A}$ is non-commutative, the possibilities (1) and (2) are again excluded because with them the identity operator would not be an irreducible tensor operator belonging to the identity corepresentation. However, with the substitution (3), the analogue of (161) is (166), which is the defining condition for a twisted irreducible tensor operator $\widetilde{Q}_{j}^{q L}$.

b. Derivations of the right coactions $\pi_{\mathcal{L}(\mathcal{A})}^{L}$ and $\tilde{\pi}_{\mathcal{L}(\mathcal{A})}^{L}$

The left regular analogues of $(\overline{\mathrm{B} 16})$ and $(\overline{\mathrm{B} 17})$ are

$$
\pi_{\mathcal{L}(\mathcal{A})}^{L \prime}(x \otimes Q)=\widehat{L}(x) Q \widehat{L}\left(x^{-1}\right)
$$

and

$$
\begin{aligned}
\pi_{\mathcal{L}(\mathcal{A})}^{L \prime}(x \otimes Q)= & (\widehat{M} \circ(i d \otimes \widehat{M}) \circ(\widehat{L} \otimes i d \otimes \widehat{L}) \circ \\
& \left.\circ(i d \otimes \sigma) \circ\left(i d \otimes S_{\mathcal{A}^{\prime}} \otimes i d\right) \circ\left(\Delta_{\mathcal{A}^{\prime}} \otimes i d\right)\right)(x \otimes Q),
\end{aligned}
$$

where $\pi_{\mathcal{L}(\mathcal{A})}^{L \prime}$ is a left action of $\mathcal{A}^{\prime}$ with carrier space $\mathcal{L}(\mathcal{A})$. However, by (A17) and (B18), and with the basis of $\mathcal{A}^{\prime}$ defined above, 


$$
\widehat{L}\left(a^{m}\right)=\sum_{j, k, \ell=1}^{g} \mu_{k}^{\ell j} s_{\ell}^{m} \mathcal{P}_{j}^{k}
$$

for all $m=1,2 \ldots, g$. On substituting (B29) into (B28), and using (A2) and (A3), it follows that

$$
\pi_{\mathcal{L}(\mathcal{A})}^{L \prime}\left(a^{m} \otimes Q\right)=\sum_{i, j, k, \ell, n, u, v, w=1}^{g}\left(m_{w u}^{v} s_{v}^{m} s_{n}^{w} \mu_{i}^{u j} \mu_{k}^{n \ell} q_{\ell}^{i}\right) \mathcal{P}_{j}^{k},
$$

for all $Q \in \mathcal{L}(\mathcal{A})$ and all $m=1,2 \ldots, g$.

Then, using (A8), the corresponding right coaction $\pi_{\mathcal{L}(\mathcal{A})}^{L}$ of $\mathcal{A}$ with the same carrier space $\mathcal{L}(\mathcal{A})$ is given by

$$
\pi_{\mathcal{L}(\mathcal{A})}^{L}(Q)=\sum_{m=1}^{g} \pi_{\mathcal{L}(\mathcal{A})}^{L \prime}\left(a^{m} \otimes Q\right) \otimes a_{m}
$$

for all $Q \in \mathcal{L}(\mathcal{A})$. Thus

$$
\pi_{\mathcal{L}(\mathcal{A})}^{L}(Q)=\sum_{i, j, k, \ell, m, n, u, v, w=1}^{g}\left(m_{w u}^{v} s_{v}^{m} s_{n}^{w} \mu_{i}^{u j} \mu_{k}^{n \ell} q_{\ell}^{i}\right)\left(\mathcal{P}_{j}^{k} \otimes a_{m}\right),
$$

for all $Q \in \mathcal{L}(\mathcal{A})$. The final stage is to re-express this in a basis free form, which can be done by writing

$$
\pi_{\mathcal{L}(\mathcal{A})}^{L}(Q)=\sum_{[Q]} Q_{[1]} \otimes Q_{[2]}
$$

where $Q_{[1]} \in \mathcal{L}(\mathcal{A})$ and $Q_{[1]} \in \mathcal{A}$ are such that

$$
\sum_{[Q]} Q_{[1]}(a) \otimes Q_{[2]}=(\sigma \circ(S \otimes i d) \circ(M \otimes i d) \circ(i d \otimes \Delta) \circ(S \otimes Q) \circ \Delta)(a)
$$

for all $Q \in \mathcal{L}(\mathcal{A})$ and all $a \in \mathcal{A}$.

For the corresponding twisted coaction $\tilde{\pi}_{\mathcal{L}(\mathcal{A})}^{L}$ the argument is similar. With the substitutions $\Delta_{\mathcal{A}^{\prime}} \rightarrow \sigma \circ \Delta_{\mathcal{A}^{\prime}}$ and $S_{\mathcal{A}^{\prime}} \rightarrow S_{\mathcal{A}^{\prime}}^{-1}$ (

$$
\begin{aligned}
\tilde{\pi}_{\mathcal{L}(\mathcal{A})}^{L \prime}(x \otimes Q)= & (\widehat{M} \circ(i d \otimes \widehat{M}) \circ(\widehat{L} \otimes i d \otimes \widehat{L}) \circ \\
& \left.\circ(i d \otimes \sigma) \circ\left(i d \otimes S_{\mathcal{A}^{\prime}}^{-1} \otimes i d\right) \circ\left(\left(\sigma \otimes \Delta_{\mathcal{A}^{\prime}}\right) \otimes i d\right)\right)(x \otimes Q),
\end{aligned}
$$

where $\tilde{\pi}_{\mathcal{L}(\mathcal{A})}^{L \prime}$ is another left action of $\mathcal{A}^{\prime}$ with carrier space $\mathcal{L}(\mathcal{A})$. By $(\mathbb{B} 29)$ and $(29)$, this gives 


$$
\tilde{\pi}_{\mathcal{L}^{\prime}(\mathcal{A})}^{L \prime}\left(a^{m} \otimes Q\right)=\sum_{i, j, k, \ell, n, u, v=1}^{g}\left(m_{n v}^{m} s_{u}^{v} \mu_{i}^{u j} \mu_{k}^{n \ell} q_{\ell}^{i}\right) \mathcal{P}_{j}^{k}
$$

for all $Q \in \mathcal{L}(\mathcal{A})$ and all $m=1,2 \ldots, g$. Using (A8), the corresponding right coaction $\tilde{\pi}_{\mathcal{L}(\mathcal{A})}^{L}$ of $\mathcal{A}$ with the same carrier space $\mathcal{L}(\mathcal{A})$ is given by

$$
\tilde{\pi}_{\mathcal{L}(\mathcal{A})}^{L}(Q)=\sum_{m=1}^{g} \tilde{\pi}_{\mathcal{L}(\mathcal{A})}^{L \prime}\left(a^{m} \otimes Q\right) \otimes a_{m}
$$

for all $Q \in \mathcal{L}(\mathcal{A})$. Thus

$$
\tilde{\pi}_{\mathcal{L}(\mathcal{A})}^{L}(Q)=\sum_{i, j, k, \ell, m, n, u, v=1}^{g}\left(m_{n v}^{m} s_{u}^{v} \mu_{i}^{u j} \mu_{k}^{n \ell} q_{\ell}^{i}\right)\left(\mathcal{P}_{j}^{k} \otimes a_{m}\right)
$$

for all $Q \in \mathcal{L}(\mathcal{A})$. This can be re-expressed in a basis free form by writing

$$
\tilde{\pi}_{\mathcal{L}(\mathcal{A})}^{L}(Q)=\sum_{[Q]} Q_{[1]} \otimes Q_{[2]}
$$

where $Q_{[1]} \in \mathcal{L}(\mathcal{A})$ and $Q_{[1]} \in \mathcal{A}$ are such that

$$
\sum_{[Q]} Q_{[1]}(a) \otimes Q_{[2]}=((i d \otimes M) \circ(\sigma \otimes S) \circ(i d \otimes \sigma) \circ(i d \otimes \Delta) \circ(i d \otimes Q) \circ \Delta)(a)
$$

for all $Q \in \mathcal{L}(\mathcal{A})$ and all $a \in \mathcal{A}$.

The right coactions $\pi_{\mathcal{T}(\mathcal{A})}^{L}$ and $\widetilde{\pi}_{\mathcal{T}(\mathcal{A})}^{L}$ of Sections VI.C.1 and VI.C.2 have essentially the same definitions as $\pi_{\mathcal{L}(\mathcal{A})}^{L}$ and $\widetilde{\pi}_{\mathcal{L}(\mathcal{A})}^{L}$, except that their domains are restricted to the appropriate subspaces $\mathcal{T}(\mathcal{A})$.

\section{APPENDIX C: PROJECTION OPERATORS}

The right regular formalism will be considered first. If $\boldsymbol{\Gamma}^{p}$ is a unitary irreducible representation of dimension $d_{p}$ of a finite group $\mathcal{G}$ of order $g$, the projection operators in the right regular formalism are defined by

$$
\mathcal{P}_{m n}^{p R}=\left(d_{p} / g\right) \sum_{x \in \mathcal{G}} \Gamma^{p}(x)_{m n}^{*} \widehat{R}(x)
$$

for all $m, n=1,2 \ldots, d_{p}$. This can be re-written as 


$$
\mathcal{P}_{m n}^{p R}=\left(d_{p} / g\right) \sum_{x \in \mathcal{G}} \Gamma^{p}\left(x^{-1}\right)_{n m}^{*} \widehat{R}(x)
$$

and hence, by (A10), (A14), (A11), and (B6),

$$
\left\langle y, \mathcal{P}_{m n}^{p R} f\right\rangle=\left(d_{p} / g\right) \sum_{x \in \mathcal{G}}\left\langle S_{\mathcal{A}^{\prime}}(x), \pi_{n m}^{p}\right\rangle\langle y, R(x \otimes f)\rangle
$$

for all $m, n=1,2 \ldots, d_{p}$. Here the $\pi_{n m}^{p}$ are the matrix coefficients of the corepresentation $\pi^{p}$ of $\mathcal{A}$ that is dual to $\Gamma^{p}$. Then, by (A13), for all $x, y \in \mathcal{A}^{\prime}$ and all $f \in \mathcal{A}$,

$$
\left\langle y, \mathcal{P}_{m n}^{p R} f\right\rangle=\left(d_{p} / g\right) \sum_{x \in \mathcal{G}}\left\langle\left(\left(i d \otimes i d \otimes S_{\mathcal{A}^{\prime}}\right) \circ\left(i d \otimes \Delta_{\mathcal{A}^{\prime}}\right)\right)(y \otimes x),\left(\pi_{\mathcal{A}}^{R}(f) \otimes \pi_{n m}^{p}\right)\right\rangle,
$$

and so, by (52) and (72),

$$
\left\langle y, \mathcal{P}_{m n}^{p R} f\right\rangle=\left(d_{p} / g\right) \sum_{x \in \mathcal{G}}\left\langle(y \otimes x),((i d \otimes M) \circ(i d \otimes i d \otimes S))\left(\pi_{\mathcal{A}}^{R}(f) \otimes \pi_{n m}^{p}\right)\right\rangle .
$$

But the Haar functional is such that

$$
h(a)=(1 / g) \sum_{x \in \mathcal{G}}\langle x, a\rangle
$$

for all $a \in \mathcal{A}$, so (C1), (83), and (52) imply that

$$
\mathcal{P}_{m n}^{p R} f=d_{p} \sum_{[f]} f_{[1]}^{R} h\left(M\left(f_{[2]}^{R} \otimes \pi_{m n}^{p *}\right)\right),
$$

As multiplication is commutative in this special case, this could equally well be written as

$$
\mathcal{P}_{m n}^{p R} f=d_{p} \sum_{[f]} f_{[1]}^{R} h\left(M\left(\pi_{m n}^{p *} \otimes f_{[2]}^{R}\right)\right)
$$

for all $m, n=1,2 \ldots, d_{p}$. In the general case the two formulae (C2) and (C3) are different, but the arguments given in Subsection IV.B show that (C3) (i.e. (107)) is actually the correct choice.

The argument in the left regular formalism follows exactly the same line, and can be obtained by merely replacing the label $R$ by $L$ at each stage. 


\section{REFERENCES}

${ }^{1}$ L. C. Biedenharn, and M.Tarlini, Lett. Math. Phys. 20, 271 (1990).

${ }^{2}$ M. Nomura, J. Phys. Soc. Japan 59, 439 (1990).

${ }^{3}$ M. Nomura, J. Phys. Soc. Japan 59, 2345 (1990).

${ }^{4}$ F. Pan, J. Phys. A24, L803 (1991).

${ }^{5}$ K. Bragiel, Lett. Math. Phys. 21, 181 (1991).

${ }^{6}$ A. U. Klimyk, Yu. F. Smirnov, and B. Gruber, in Symmetries in Science. V. Algebraic systems, their representations, realizations, and physical applications edited by B. Gruber, L. C. Biedenharn, and H.D. Doebner, (Plenum Press, New York, 1991) p. 341.

${ }^{7}$ Yu. F. Smirnov, V. N. Tolstoy, and Yu. I. Kharitonov, in Symmetries in Science. V. Algebraic systems, their representations, realizations, and physical applications edited by B. Gruber, L. C. Biedenharn, and H.D. Doebner, (Plenum Press, New York, 1991) p. 487.

${ }^{8}$ V. Rittenberg, and M. Scheunert, J. Math. Phys. 33, 436 (1992).

${ }^{9}$ A. U. Klimyk, J. Phys. A25, 2919 (1992).

${ }^{10}$ M. Gould, and L. C. Biedenharn, J. Math. Phys. 33, 3613 (1992).

${ }^{11}$ L. C. Biedenharn, and M. A. Lohe, in Quantum Groups edited by P. P. Kulish, Lecture Notes in Mathematics, Vol. 1510 (Springer-Verlag, Berlin, 1992) p. 197.

${ }^{12}$ C. Quesne, Phys. Lett. B298, 344 (1993).

${ }^{13}$ C. Quesne, Phys. Lett. B304, 81 (1993).

${ }^{14}$ N. Aizawa, J. Math. Phys. 34, 1937 (1993).

${ }^{15}$ L. C. Biedenharn, M. A. Lohe, and H. T. Williams, J. Math. Phys. 35, 6672 (1995).

16 J. F. Cornwell, Group Theory in Physics, Vol. I. (Academic Press, London ,1984).

17 J. F. Cornwell, Group Theory in Physics, Vol. II. (Academic Press, London ,1984).

${ }^{18}$ E. P. Wigner, Gruppentheorie und ihre Anwendung auf die Quantenmechanik der Atomspektren (Friedr. Vieweg und Sohn, Braunschweig, 1931).

19 S. L. Woronowicz, Comm. Math. Phys. 111, 613 (1987).

${ }^{20}$ S. L. Woronowicz, Invent. Math. 93, 35 (1988). 
21 S. L. Woronowicz, Lett. Math. Phys. 21, 35 (1991).

${ }^{22}$ M. S. Dijkhuizen, and T. H. Koornwinder, Lett. Math. Phys. 32, 315 (1994).

${ }^{23}$ M. S. Dijkhuizen, and T. H. Koornwinder, Geometriae Dedicata 52, 291 (1994).

${ }^{24}$ M. S. Dijkhuizen, On compact quantum groups and quantum homogeneous spaces, doctoral thesis, University of Amsterdam (1994).

25 T. H. Koornwinder, General compact quantum groups, a tutorial, University of Amsterdam, Math. preprint 94-06 (1994).

26 T. H. Koornwinder, in General compact quantum groups and q-special functions, edited by V. Baldoni and M. A. Picardello, Pitman Research Notes in Maths. (Longman, London, 1994) p. 46.

27 M. E. Sweedler, Hopf algebras (Benjamin, New York ,1969).

28 S. Majid, Int. J. Mod. Phys. A5, 1 (1990).

${ }^{29}$ V. Chari, and A. Pressley, Quantum groups (Cambridge Univ. Press, Cambridge, 1994).

30 V. G. Drinfel'd, Leningrad Math. J. 1, 321 (1990).

31 P. Podleś, Lett. Math. Phys. 99, 193-202 (1987).

${ }^{32}$ M.Noumi, H. Yamada, and K. Mimachi, Proc. Japan Acad. Ser. A. Math. Sci. 65, 169$171(1989)$.

33 L. L. Vaksman, and Y. S. Soibel'man, Leningrad Math. J. 2, 1023-1043 (1991).

34 T. Masuda, K. Mimachi, Y. Nakagami, M.Noumi, Y. Saburi, and K. Ueno, J. Funct. Anal. 99, 127-151 (1991).

35 M. Noumi, and K. Mimachi, in Quantum Groups, edited by P. P. Kulish, Lecture Notes in Mathematics, Vol. 1510 (Springer-Verlag, Berlin, 1992) p. 98- 103.

36 M.Noumi, H. Yamada, and K. Mimachi, Japan. J. Math. 19, 31-80 (1993).

37 S. Majid, in Quantum groups, integrable statistical models, and knot theory edited by M. L. Ge and H. J. de Vega (World Scientific, Singapore, 1993) p. 231. 